\title{
Design and Operation of Automated Ice-Tethered Profilers for Real-Time Seawater Observations in the Polar Oceans
}

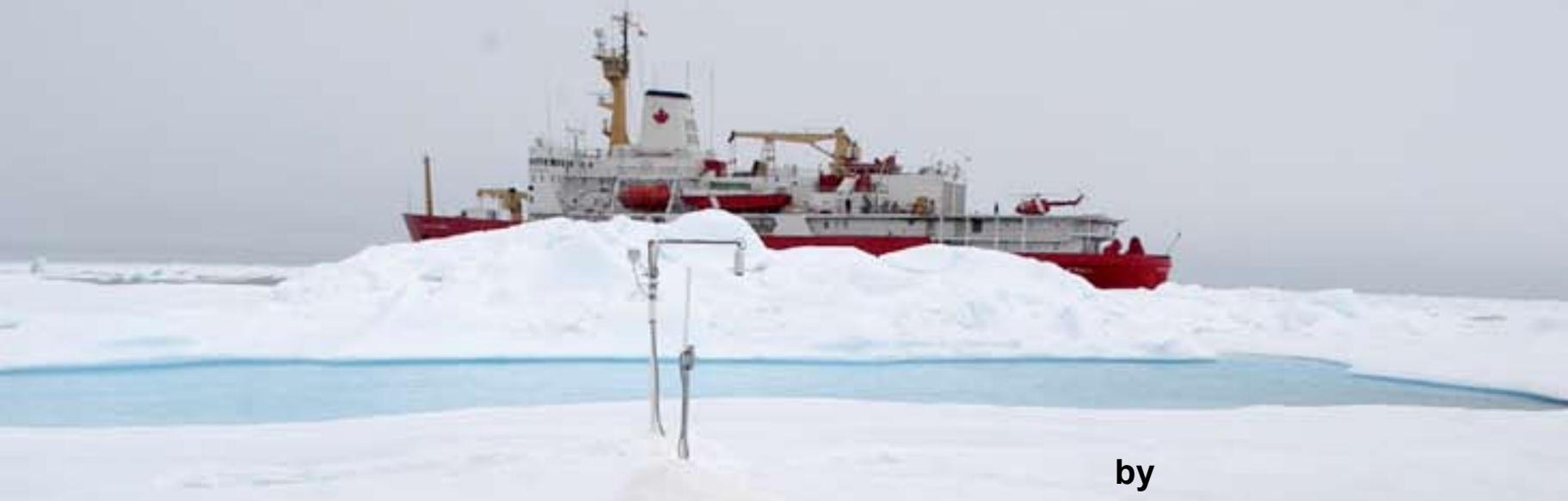

Krishfield, R., K. Doherty, D. Frye,
T. Hammar, J. Kemp, D. Peters,
A. Proshutinsky, J. Toole, and
K. von der Heydt
Woods Hole Oceanographic Institution
Woods Hole, MA 02543
June 2006
Funding was provided by the National
Science Foundation under
Contract Nos. OCE-0324233
and ARC-0519899.
Approved for public release;
Distribution unlimited.




\section{WHOI-2006-11}

Ice-Tethered Profilers for Real-Time Seawater Observations in the Polar Oceans

by

Krishfield, R., K. Doherty, D. Frye, T. Hammar, J. Kemp, D. Peters, A. Proshutinsky, J. Toole, and K. von der Heydt

June 2006

\section{Technical Report}

Funding was provided by the National Science Foundation throough Grant Nos. OCE-0324233 and ARC-0519899.

Reproduction in whole or in part is permitted for any purpose of the United States Government. This report should be cited as Woods Hole Oceanog. Inst. Tech. Rept., WHOI-2006-11.

Approved for public release; distribution unlimited.

Approved for Distribution:

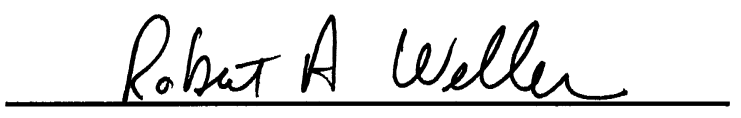

Robert A. Weller, Chair

Department of Physical Oceanography 
Cover photo: An Ice Based Observatory (IBO) consisting of the surface package of Ice-Tethered Profiler (ITP) number 1 in front of Ice Mass Balance (IMB) buoy 7949 after installation at $79.8^{\circ} \mathrm{N}, \mathbf{1 5 0}^{\circ} \mathrm{W}$ in August 2005 with CCGS Louis S. St. Laurent in the background (photo by Chris Linder). 


\section{TABLE OF CONTENTS}

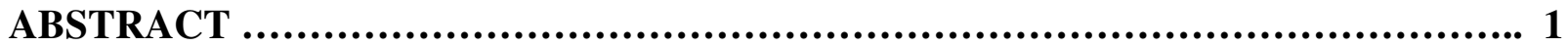

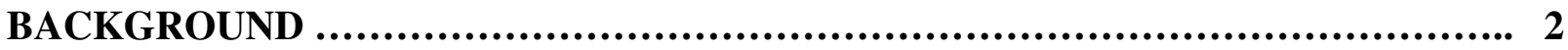

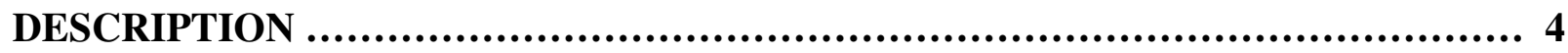

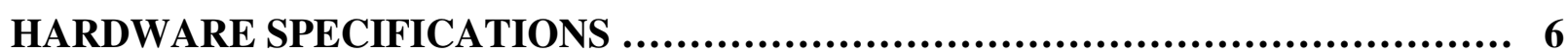

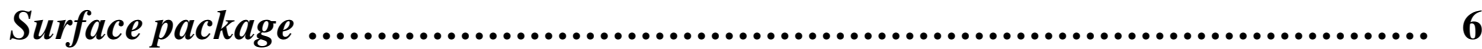

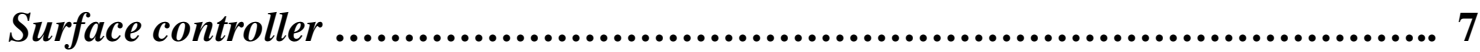

Inductive modem ........................................................................ 8

Iridium transceiver ................................................................... 9

GPS receiver ......................................................................... 10

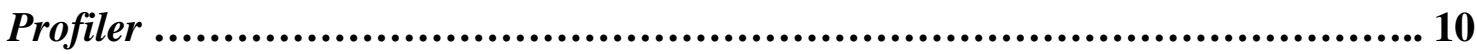

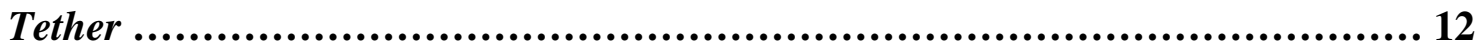

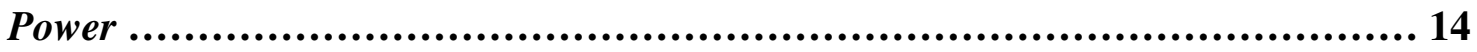

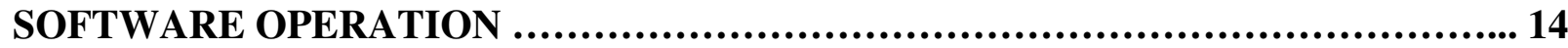

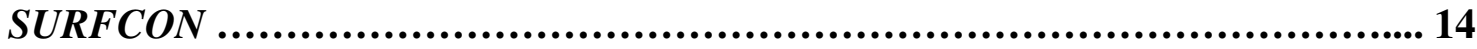

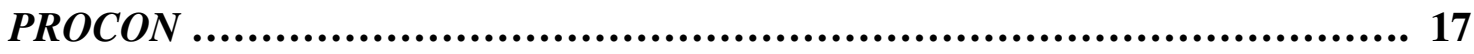

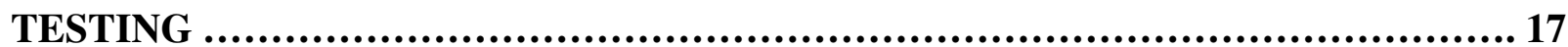

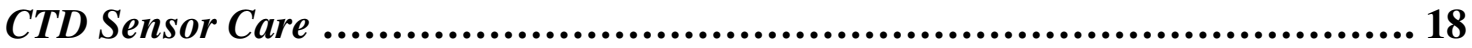

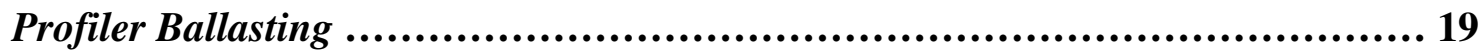

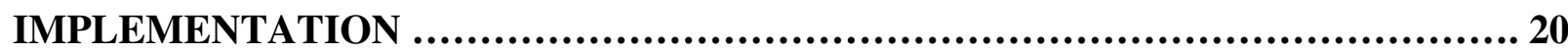

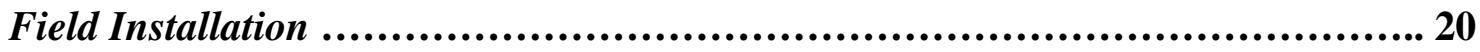

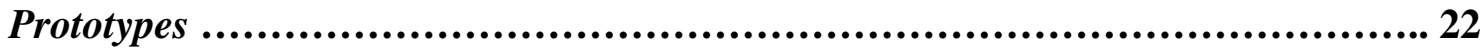

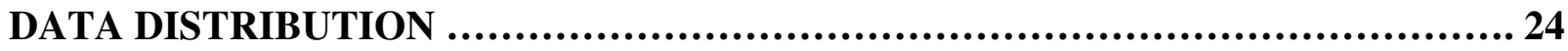

Iridium Upload ................................................................... 24

Preliminary Processing ........................................................... 25

Sample Data from First ITP .................................................... 26

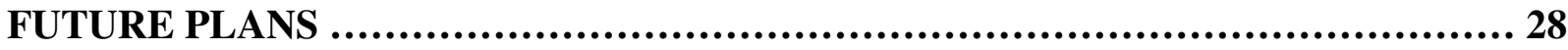

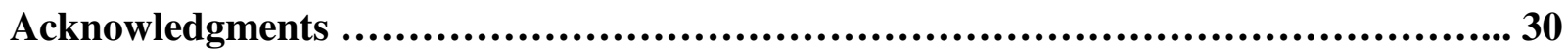

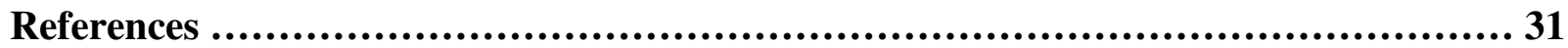

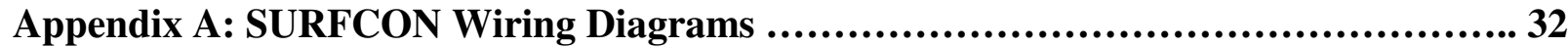

Appendix B: ITP Profiler Wiring Connections …........................................ 34

Appendix C: Operating the ITP surface package ....................................... 36

Appendix D: Assembly of ITP Profiler ................................................. 48

Appendix E: CTD Configuration for PROCON …...................................5 50

Appendix F: Operating the Profiler .....................................................5 52

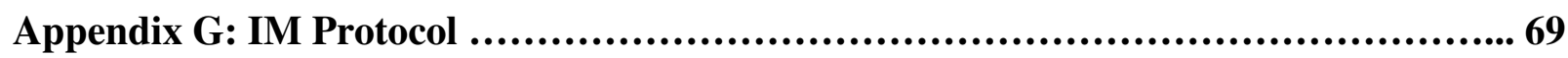

Appendix H: SURFCON to Logger Communications ..................................... 73 


\section{LIST OF FIGURES}

Figure 1: Ice-Tethered Profiler Schematic ................................................. 3

Figure 2: ITP Electronic Diagram ......................................................... 4

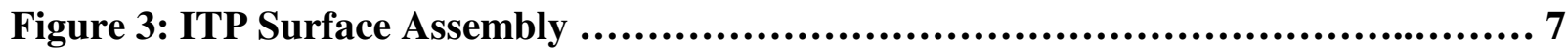

Figure 4: SURFCON board, Persistor CF-2 computer, and SeaBird SIM mounted in ITP surface electronics chassis ....................................................... 8

Figure 5: NAL Iridium transceiver and antenna on ITP electronics chassis ................ 9

Figure 6: ITP Profiler Unit ............................................................ 10

Figure 7: Attaching ITP to mooring wire during deployment ........................... 11

Figure 8: ITP Cable Assembly, upper 5 m of 805 m segment ............................ 13

Figure 9: SURFCON operation block diagram ......................................... 16

Figure 10: Temperature, voltage, and locations from ITP1 and ITP3 cold, dock, and field

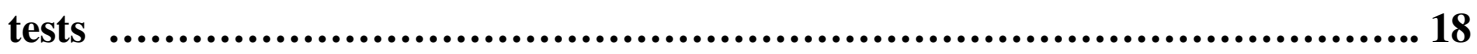

Figure 11: Transporting ITP gear to deployment site using hand sleds and helicopters . 21

Figure 12: Augering 11" deployment hole with tripod assistance .......................... 22

Figure 13: ITP prototype drift tracks ................................................ 23

Figure 14: Profile-averaged motor currents versus time …............................... 26

Figure 15: A subset of the CTD data from the prototype ITP ............................ 27

Figure 16: Expanded views of the potential temperature profiles and potential temperature - salinity diagrams ................................................................ 27

Figure 17: Simulated drift tracks for one year from 20 ITPs deployed in the Arctic at the circles and forced with climatological ice drift vectors from the IABP ............ 28 


\title{
Design and Operation of Automated Ice-Tethered Profilers for Real-time Seawater Observations in the Polar Oceans.
}

\begin{abstract}
An automated, easily-deployed Ice-Tethered Profiler (ITP) has been developed for deployment on perennial sea ice in polar oceans to measure changes in upper ocean temperature and salinity in all seasons. The ITP system consists of three components: a surface instrument that sits atop an ice floe, a weighted, plastic-jacketed wire-rope tether of arbitrary length (up to $800 \mathrm{~m}$ ) suspended from the surface instrument, and an instrumented underwater unit that profiles up and down the wire tether. The profiling underwater unit is similar in shape and dimension to an ARGO float except that the float's variable-buoyancy system is replaced with a traction drive unit. Deployment of ITPs may be conducted either from ice camps or icebreakers, utilizing a self contained tripod/winch system that requires no power. Careful selection of an appropriate multiyear ice floe is needed to prolong the lifetime of the system (up to 3 years depending on the profiling schedule). Shortly after deployment, each ITP begins profiling the water column at its programmed sampling interval. After each acquired temperature and salinity profile, the underwater unit (PROCON) transfers the data and engineering files using an inductive modem to the surface controller (SURFCON). SURFCON also accumulates battery voltages, buoy temperature, and locations from GPS at specified intervals in status files, and queues that information for transmission at the start of each new day. At frequent intervals, an Iridium satellite transceiver in the surface package calls and transmits queued status and CTD data files onto a WHOI logger computer, which are subsequently processed and displayed in near-real time at http://www.whoi.edu/itp. In 2004 and 2005, three ITP prototypes were deployed in the Arctic Ocean. Each system was programmed with accelerated sampling schedules of multiple one-way traverses per day between 10 and 750-760 m depth in order to quickly evaluate endurance and component fatigue. Two of the ITPs are continuing to function after more than 10 months and 1200 profiles. Larger motor currents are observed at times of fast ice floe motion when larger wire angles develop and drag forces on the profiler are increased. The CTD profile data so far obtained document interesting spatial variations in the major water masses of the Beaufort Gyre, show the double-diffusive thermohaline staircase that lies above the warm, salty Atlantic Layer, and many mesoscale eddys. Deployed together with CRREL Ice Mass Balance (IMB) buoys, these ITP systems also operate as part of an Ice Based Observatory (IBO). Data returned from an array of IBOs within an Arctic Observing Network will provide valuable real time observations, support studies of ocean processes, and facilitate numerical model initialization and validation.
\end{abstract}




\section{BACKGROUND}

The need for broad-scale, frequent ocean sampling is being addressed outside the polar latitudes by the JASON (http://sealevel.jpl.nasa.gov/mission/jason-1.html) and Argo (http://www.argo.ucsd.edu/) projects that are returning satellite altimeter measurements of sea level anomalies and drifting profiling float measurements of the upper ocean temperature and

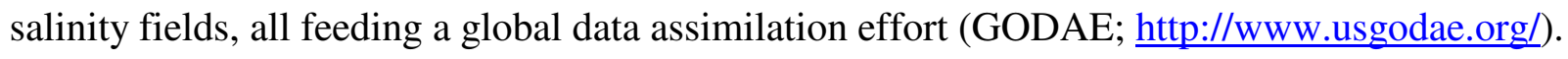
Since perennial sea ice makes it difficult to use many modern automated observational instruments, the Arctic represents an observational gap in the "global" ocean observing system. Consequently, there are not sufficient observations to accurately quantify change in environmental conditions and to validate or disprove hypotheses about Arctic climate variability.

Those observations that have been made hint that the Arctic has been undergoing large changes recently, motivating broad, interdisciplinary, multi-scale research (SEARCH SSC, 2001). For instance, changes in the Atlantic water, and in the overlying cold halocline layer have been observed over the previous decades (e.g. Morison et al., 2000). At the same time, an ensemble of global climate models predicts the decline and eventual disappearance of the perennial sea ice cover in the Arctic within this century (ACIA, 2004). These concerns have led to enhanced international research in the Arctic, including organization of an International Polar Year (IPY) in 2007-2008.

SEARCH developed a measurement strategy in recognition that a number of long-term, large-scale observing systems have been terminated, or are in danger of disappearing. One of the four major SEARCH themes is thus long-term observations to detect and monitor environmental change. This has spurred various recent workshops to devise national and international Arctic observing networks that integrate many types of instruments (such as buoys, under-ice floats, AUVs, moorings and cabled observatories) to achieve broad coverage of the Arctic Ocean (e.g. Proshutinsky et al., 2004). Internationally, an integrated Arctic Ocean Observing System (iAOOS; Dickson et al., 2006) has been endorsed by the Arctic Ocean Science Board. The US Polar Research Board has also recently discussed this concept in the National Academy of Sciences report: "Towards an Integrated Arctic Observing Network" (IAON); (available from http://www.nap.edu/catalog/11607.html).

One of the observational tools recognized as fundamental in observing networks are IceBased Observatories (IBOs) which are defined as "automated, drifting, ice-based sensor systems providing comprehensive data from the Arctic environment and incorporating the multidisciplinary needs of biological, chemical and physical oceanography, as well as different aspects of atmospheric and sea-ice studies" (Proshutinsky et al., 2004). Ice-tethered drifters with discrete subsurface instrumentation such as the SALARGOS (Morison et al., 1982), IOEB 
(Honjo et al., 1995) and J-CAD (Kikuchi et al., 2002) buoys, have been successfully fielded in the Arctic in the past two decades, demonstrating that automated buoys are viable means of acquiring long-term, in situ data from beneath the ice pack. However, the vertical resolution of the temperature and salinity observations from these systems has typically been limited to only a few depths due to the costs associated with outfitting multiple sensors on a single package. In addition, intercalibration of these strings of multiple sensors can be difficult. Moreover, even with a limited number of sensors, total system costs has meant that only a small number of such devices have been fielded at any one time. Another shortcoming of the ocean measurements from those systems is that limited vertical resolution potentially misses some of the small-scale ocean structures of the Arctic Ocean.

Technological and engineering improvements have fostered the development of a number of IBOs consisting of clusters of meteorological, ice mass balance (IMB), and discrete-depth sampling ocean buoys (one example being the North Pole Environmental Observatory, NPEO; http://psc.apl.washington.edu/northpole/). To address the need for automated ocean profiling under sea-ice as part of an IBO, and be both useful and practical to the Arctic scientific community, an instrument with the following specifications was proposed:

- Capable of returning in real time, better than 1-m-vertical-resolution, highaccuracy daily (or semi-daily) temperature and salinity profiles to $750 \mathrm{~m}$ depth for 3 years (assuming deployment in robust icefloes).

- Deployable from light aircraft (Twin Otters) and helicopters through a conventional 10.5" ice-augered hole.

- $\quad$ Modestly priced, allowing them to be deployed in large numbers and be considered expendable.

- Capable of supporting plug-in modules for additional surface and/or subsurface instrumentation.

Building on the success of ice drifters, and on the WHOI-developed Moored Profiler instrument (Doherty et al., 1999), an automated easily-deployed Ice-Tethered Profiler (ITP) has been developed to economically measure changes in upper ocean temperature and salinity throughout large expanses of the Arctic Ocean in all seasons (Figure 1). The nominal tether length is $800 \mathrm{~m}$, sufficient to profile through the temperature maximum layer characterizing the Atlantic waters that enter the Arctic through the Fram Strait. 


\section{DESCRIPTION}

The ITP system consists of three components: a surface instrument package that sits atop an ice floe, a weighted, wire-rope tether of arbitrary length (up to $800 \mathrm{~m}$ ) suspended from the surface package, and an instrumented underwater unit that travels up and down the wire tether.

The surface unit houses a controller, inductive modem, GPS receiver and an Iridium satellite phone with associated antennae and batteries within a water tight aluminum housing capped by an ultra high molecular weight (UHMW) polyethylene dome. The electronics case sits within a cylinder of Surlyn ionomer foam designed to provide buoyancy for the plastic-jacketed wire rope tether with ballast termination weight, and provide modest protection in the event of ice ridging, and flotation should the ice fracture.

The upper 5-m of the wire tether is cast within a thick protective urethane jacket that also houses an electrical ground lead for the inductive modem. A custom termination is used to mechanically join the tether to the surface unit and preserve the electrical isolation of the wire tether from the sea water.

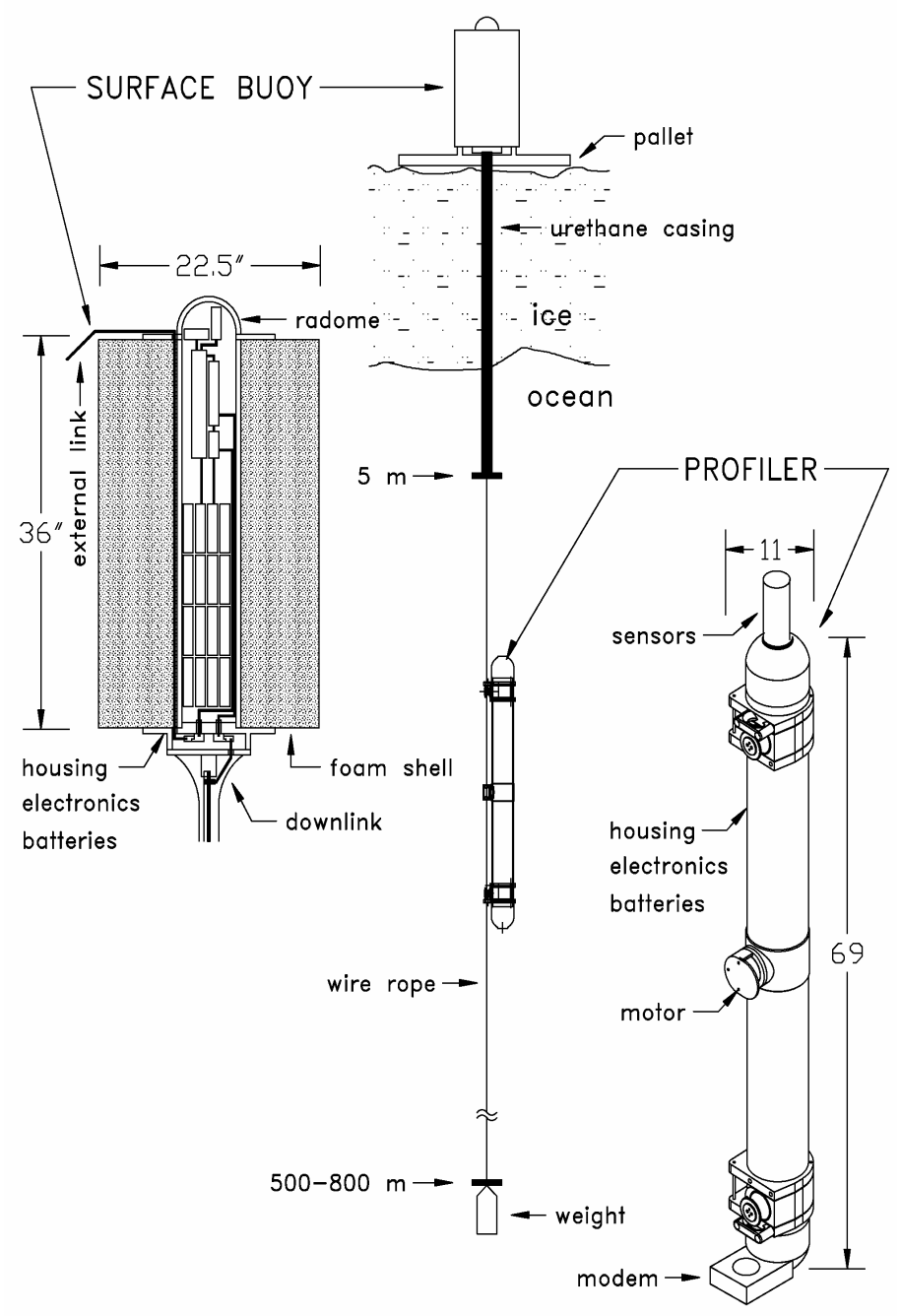

Figure 1: Ice-Tethered Profiler Schematic

A new variation of the WHOI Moored Profiler (in shape and size much like an ARGO float) mounts on this tether and cycles vertically along it. The ITP employs the same CTD sensor package that is currently used on many of the ARGO floats (the Sea Bird Electronics, Inc. model 41CP) mounted in the instrument's top hemispherical end cap. Communication between the Profiler and surface controller is supported by an inductive modem utilizing the wire tether (Figure 2). The raw CTD and associated engineering data are downloaded at the completion of each one-way profile then telemetered to shore. The buoy transmits all data in near-real time and 


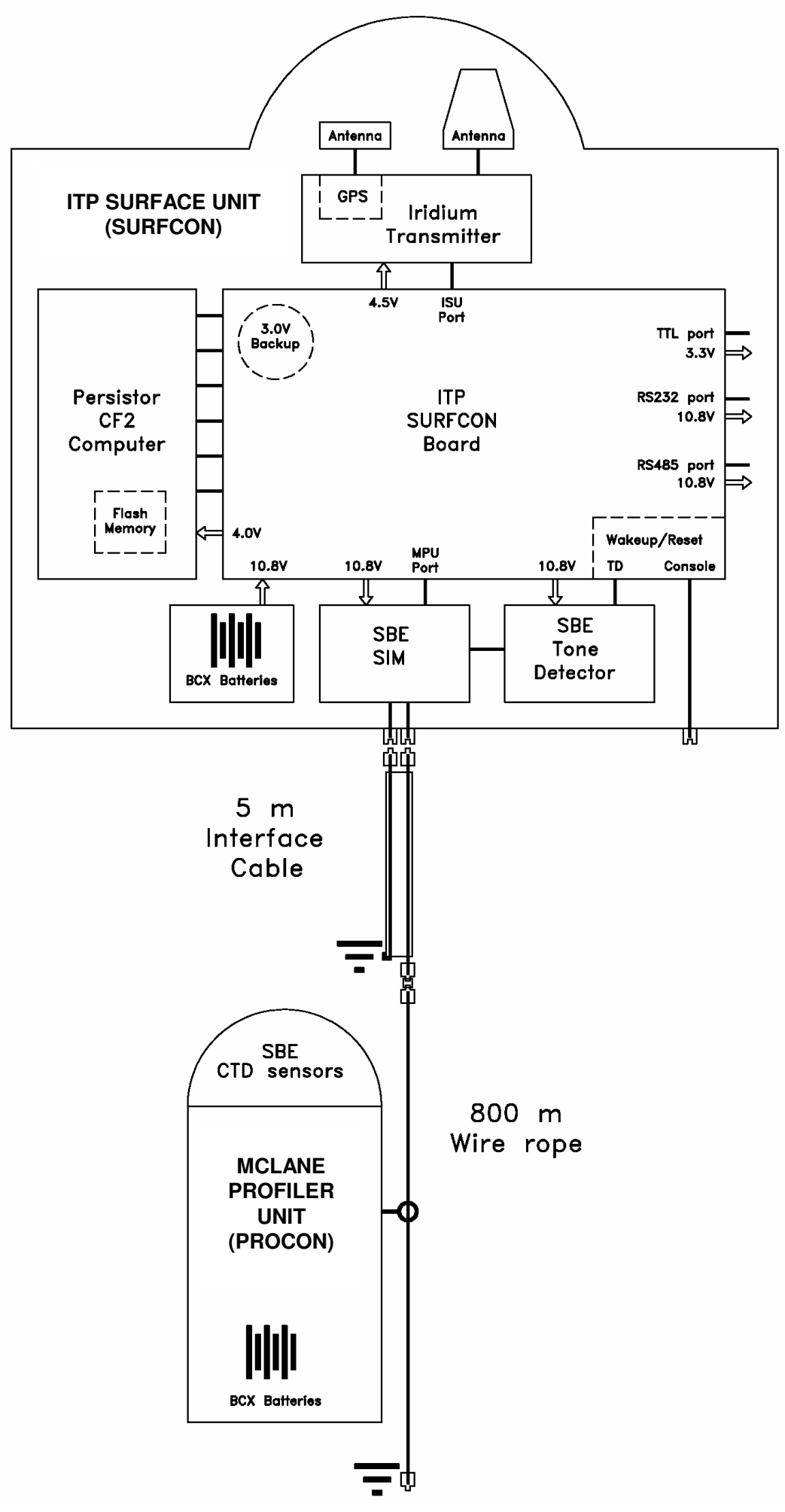

Figure 2: ITP Electronic Diagram is low-cost, allowing systems to be considered expendable (thus alleviating the need for expensive recovery operations).

The size and weight of the system and the deployment gear were minimized in order to facilitate handling and transportation. Altogether with the tether ballast weight, the ITP hardware totals approximately $1000 \mathrm{lbs}$, allowing transport to remote sites by a medium lift helicopter or Twin Otter aircraft (along with the deployment technicians, emergency survival gear, and the deployment equipment).

A total of three ITP prototypes were deployed in 2004 and 2005, together with US Army Cold Regions Research and Engineering Laboratory (CRREL) IMB buoys. Thousands of profiles later, data from these systems support the IBO concept as a practical means to return sustained observations of the Arctic air-ice-ocean environment in all seasons. 


\section{HARDWARE SPECIFICATIONS}

Mooring length: 10 to $800 \mathrm{~m}$

Profiling range: $1,500,000 \mathrm{~m}$ on standard battery pack

Duration: 2.5 to 3 years returning two $750 \mathrm{~m}$ (one way) profiles per day (2000 total)

Temperature specification: $-35^{\circ} \mathrm{C}$ for prototypes

Data telemetry: typically 50 Kbytes per profile (totaling 100 Mbytes over 3 years)

Sensors: Sea-Bird 41-CP CTD (same as many ARGO floats)

Telemetry: Sea-Bird inductive link from profiler to surface unit; Iridium link to shore

Power: lithium BCX “DD” battery packs; $3300 \mathrm{Wh}$ in surface package (after derating for temperature), $2500 \mathrm{Wh}$ in profiler Size: Profiler fits through a 10.5-1" diameter hole in the ice

\section{Surface Package}

The surface expression of the ITP (Figure 3) is a cylindrical buoy that is deployed on a multiyear ice floe. Balancing durability and disposability, the surface unit incorporates a foam collar for shock absorption in order to increase the lifetime of the drifter during ridging, and depending on size may either sink or buoy the system should the ice fracture. The surface collar (22 or 26" outer diameter) is constructed from Surlyn ionomer foam, and surrounds an aluminum electronics compartment (36" long, 6.5" diameter tube) that contains an Iridium modem, GPS receiver, inductive modem interface to underwater sensors, a data controller and lithium batteries. The Iridium and GPS antennae are mounted internally on a chassis beneath an UHMW hemispherical radome, which forms the top endcap. The bottom endcap provides space for penetrators to feed the electrical signals into the compartment, and a mount for the tether. Space for an additional bulkhead connector for interfacing with other external sensors is provided, as well as extra tube length for batteries to meet additional power requirements in the future. The yellow surface package is propped on a wooden palett during deployment (to minimize the ablation of ice in contact with the unit). 


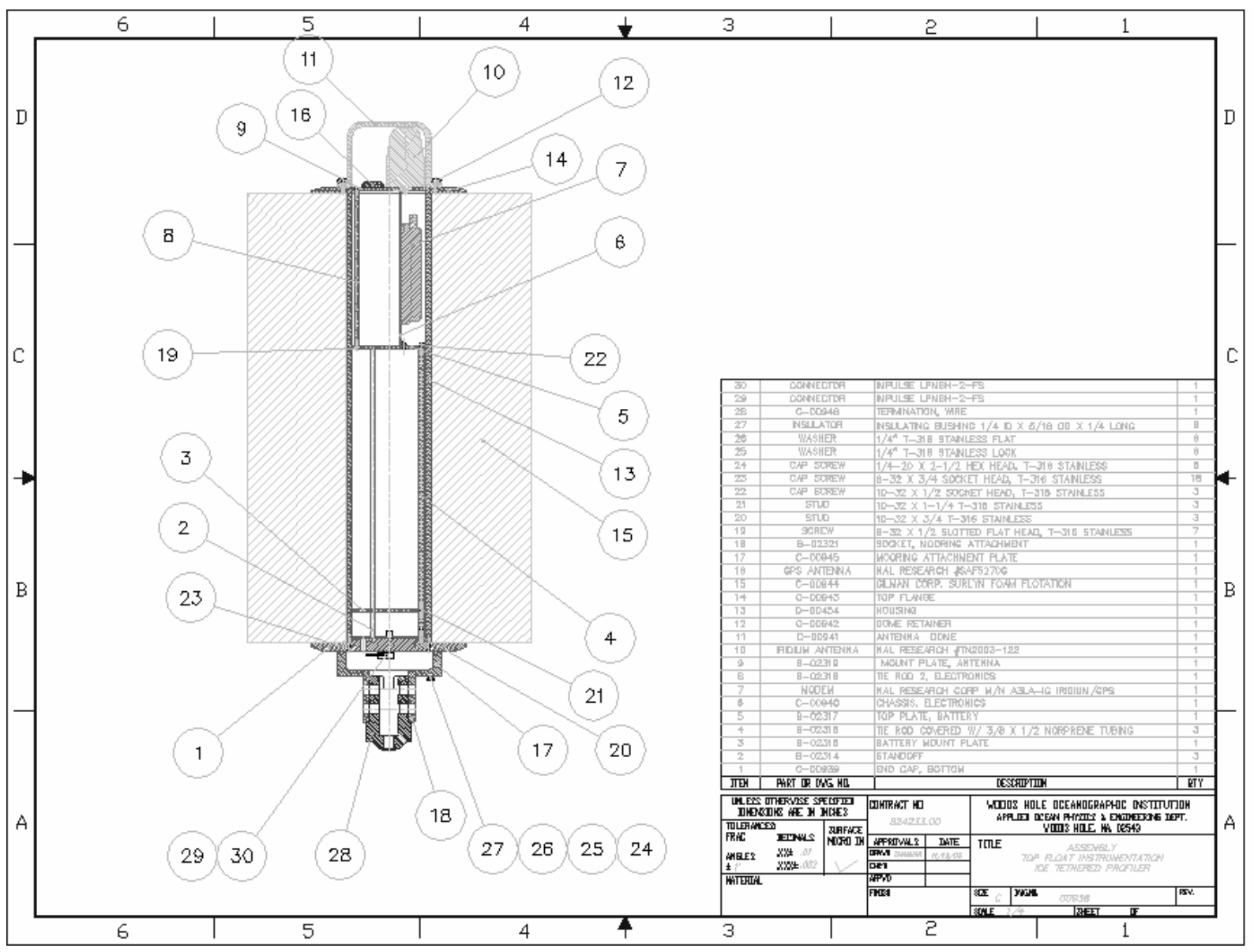

Figure 3: ITP Surface Assembly

\section{Surface controller}

The data controller in the surface package (SURFCON) operates the inductive modem interface, obtains the data from the instrument on the subsurface tether, formats and feeds the information to the Iridium satellite transmitter (Figure 4). The computer at the heart of the controller is a Persistor CF2 commercially available, low power, $\mathrm{C}$ programmable microcontroller with a $128 \mathrm{Mb}$ compact flashcard memory. The Persistor computer is only 0.7"x2"x3" in size and it and the flashcard are rated to operate to $-40^{\circ} \mathrm{C}$. The flashcard memory should be sufficient to store all acquired instrument and engineering data, status, and log files for the lifetime of the system. The SURFCON interface board was designed at WHOI to regulate power to the computer, waken upon detecting a tone from the underwater inductive modem (UIM), communicate via the surface inductive modem (SIM), acquire location information from a GPS receiver, control command and data transmissions with WHOI based servers via an Iridium satellite link (ISU), provide console access (or RS232 port), and provide spare logic and RS485 (multidrop) serial ports. 
The main UART is used to program the Persistor and communicate with the operator via the console access port. The console port is wired to a bulkhead connector on the base of the surface package for access with the battery packs installed and fully assembled. Time Processor Unit (TPU) channels are configured either as logic I/O bits or software UARTs to interface with the tone detector (TD), SIM, Iridium telephone modem, and other serial device ports (logic level, RS232, and RS485). One serial device port on the SURFCON board acquires the GPS data. The other device ports are not presently used on the ITPs, but are integrated in the design to support communications to other instruments in the future.

The TD board is a product of Sea-Bird Electronics (product number 41247), and is used to waken the SURFCON board, which will apply power to the $\mathrm{CF} 2$ computer. Non volatile storage allows the computer to resume operation where it left off when suspended.

Lithium battery $(\mathrm{BCX})$ packs at $10.8 \mathrm{~V}$ powers SURFON which converted $4.0 \mathrm{~V}$ for the $\mathrm{CF} 2$, and $4.5 \mathrm{~V}$ for the ISU. A $3 \mathrm{~V}$ backup battery for the CF2 is also included. During operation, current drain is typically less than $120 \mu \mathrm{A}$ in sleep mode (almost entirely from TD board), while the interface board draws $10 \mu \mathrm{A}$ from its backup battery. The operation of the surface controller has been tested functionally to $-48^{\circ} \mathrm{C}$, and though the Iridium unit will not transmit at that temperature, normal operations resume after warming without data loss.

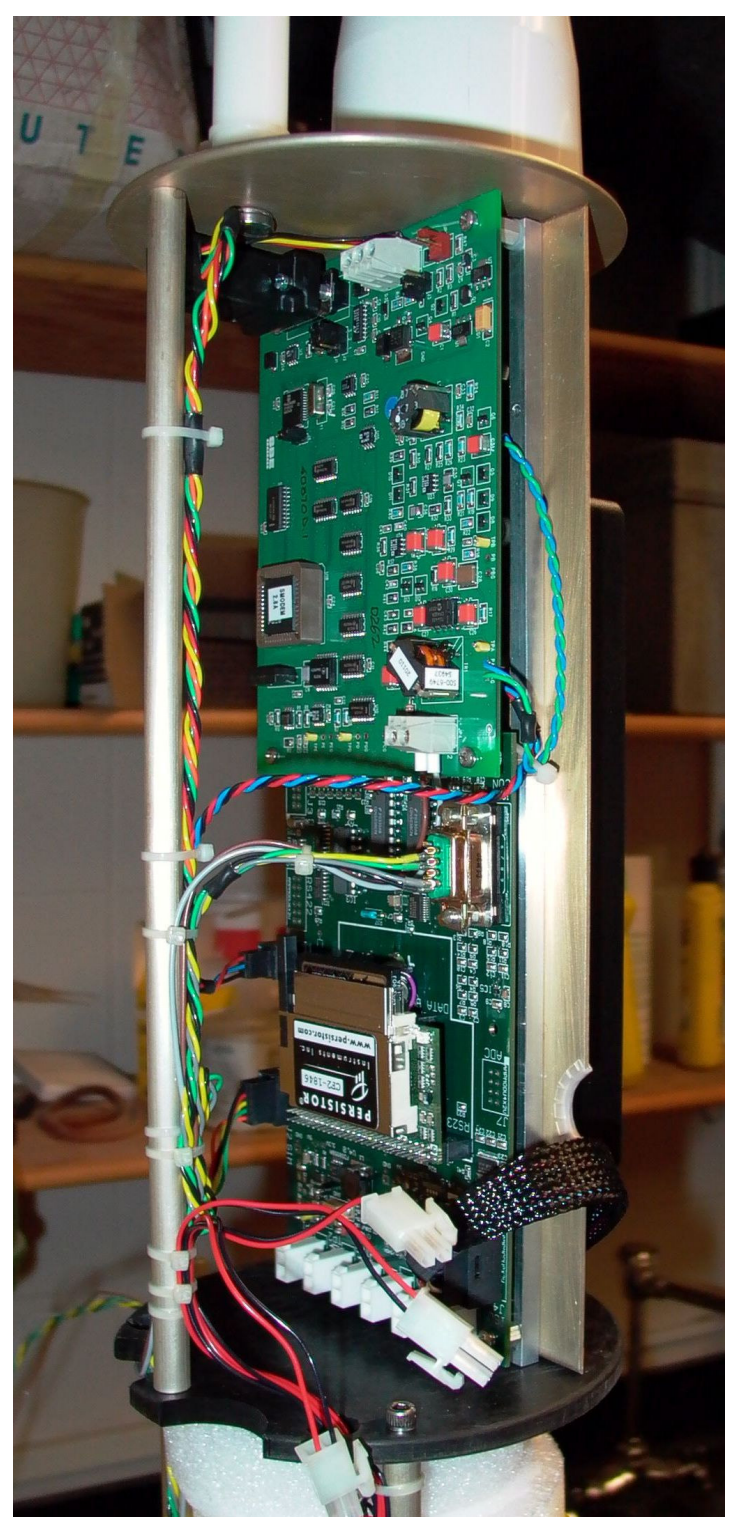

Figure 4: SURFCON board, Persistor CF-2 computer, and SeaBird SIM mounted in ITP surface electronics chassis. The TD board is mounted under the SIM board.

\section{Inductive modem}

A commercially available (Sea-Bird, Inc.) SMODEM-2 SIM (and TD board) in the surface unit (Figure 4) is electrically connected to the tether conductor and the tether passes through a corresponding modem toroid that is carried by the Profiler. The inductive circuit for 
communications is completed by grounding plates on the conductors and the seawater path. The data link from the SIM to the computer is at 9600 baud and the link to the underwater instrument is 1200 baud.

Formerly, only ASCII data could be transferred, but SeaBird agreed to upgrade their transmission protocol to allow binary data transfers. Firmware changes add a command that allows the SIM to request an 8-bit transfer from the UIM. During the binary transfers, control of the half duplex link is effected by a programmable transmission gap at both SIM and UIM ends, rather than by the special characters employed in ASCI transfer. Files of any length are sent in packets of 4 Kbytes with about a 2 second gap between each (at a nominal 1200 baud with net throughput of approximately 90 bytes/s).

It is worth noting that other devices equipped with inductive modems could also be mounted on the mooring cable and configured to communicate with the data controller.

\section{Iridium transceiver}

The Iridium 9522 LBT modem (Figure 5) dials up, logs in and uploads the profiler data, locations and status information to a dedicated computer at WHOI. The NAL model A3LA-IG modems on the first ITPs also obtained GPS fixes and that information was added to the instrument data, but the GPS receiver is separate from the modem on later ITPs.

In the ITP application, the SURFCON controller powers the modem when it is time to download data via Iridium and uses Hayes modem commands over a serial connection to check registration with the Iridium satellite (AT+CREG?), check signal strength (AT+CSQ) and dial-up (ATDT...).

The Iridium modems (or ISU) that were used on the prototypes had specified operating temperatures down to $-30^{\circ} \mathrm{C}$, but extended

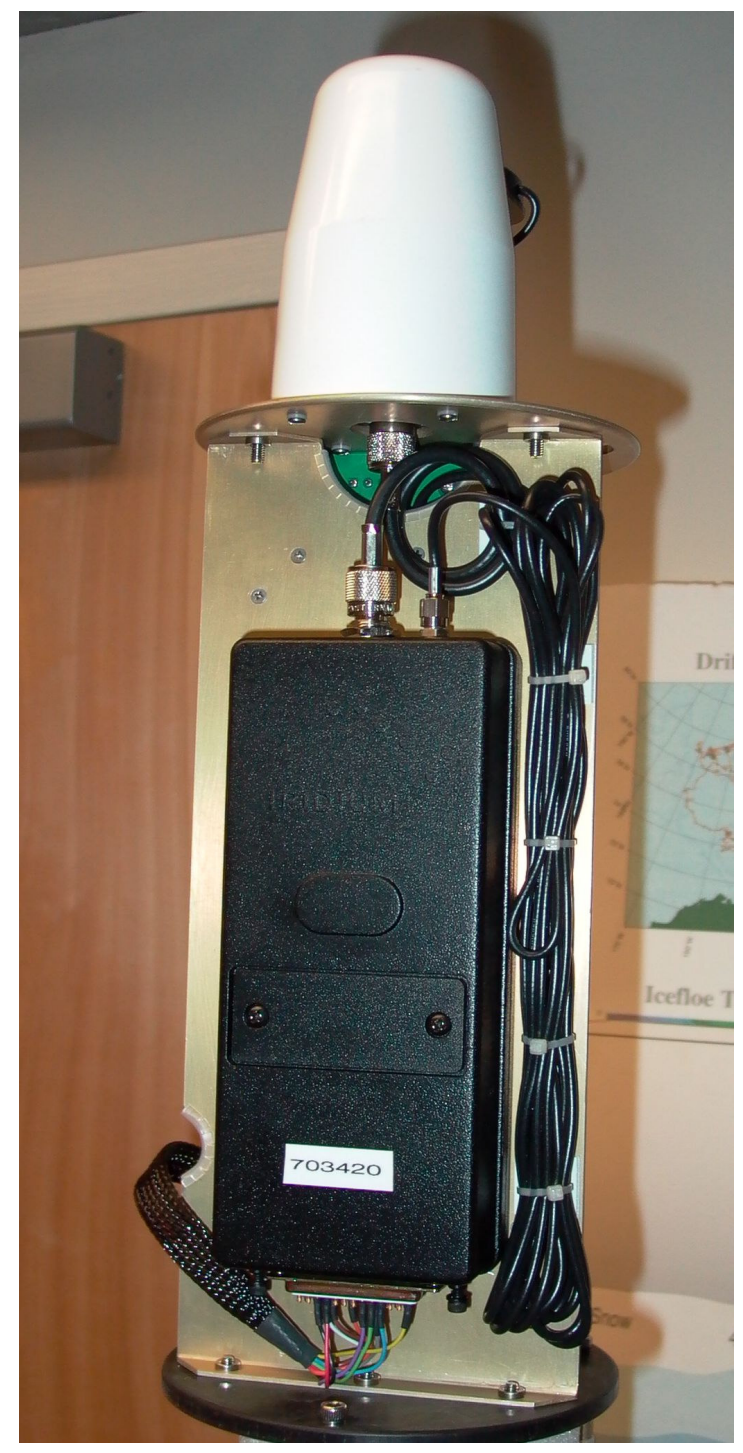

Figure 5. NAL Iridium modem and antenna on ITP electronics chassis. 
environmental testing of all the surface electronics found some that operated reliably as cold as $38^{\circ} \mathrm{C}$. However, the ISUs with integrated GPS needed an increasingly long off period as temperature decreased (as long as one hour at $-40{ }^{\circ} \mathrm{C}$ ) to assure proper functionality at power up. This did not seem to be a problem on ISUs without integrated GPS.

A "dial-up" data account with a commercial company is required for billing the Iridium telemetry charges, and a SIM card (not to be confused with the surface inductive modem) is obtained for each ITP. Besides the cost of the SIM card, telemetry charges include an activation fee, monthly charge, and per minute charges (about \$1/min in 2004) using the "Data Only" service. At current rates, the cost to transmit a typical $750 \mathrm{~m}$ ITP profile is about $\$ 4$.

The SIM cards are installed in the Iridium transmitters in each ITP surface unit and typically come with a default password ("1111") which needs to be deleted to enable dial-up capability. The Hayes commands for deactivating the default password (PIN) is: AT+CLCK= "SC",0,"1111". Change from PIN to no PIN with AT+CPIN= "1111". Response should be OK.

\section{GPS receiver}

On the first 3 ITPs, Iridium modems with integrated GPS were used. However, since the modems were not equipped with battery backup for the GPS receiver, every location had to be acquired from a cold start every time, which consumes excess time and energy. Consequently, on later ITPs separate Navman Jupiter 21 GPS receivers are connected to an unused logic level port on the SURFCON board, and provided with a separate back up battery to store locations between acquisitions, thus reducing the time and energy required for a location fix.

\section{Profiler}

The Profiler unit (Figure 6) adapts elements of the operational ARGO float and Moored Profiler instruments. The cylindrical pressure case houses the batteries, drive system, instrument controller, CTD and underwater inductive modem (UIM) as well as provides buoyancy for the neutrally buoyant unit. The instrument controller and software are products of McLane Research Laboratories, Inc. and use the same electronic hardware and software scheme as the commercially available McLane Moored Profiler (MMP; Morrison et al., 2000). Hardware wiring diagrams are given in Appendix B, profiler assembly instructions in Appendix D, and detailed operating instructions in Appendix F.

Taking advantage of the extensive CTD development effort for ARGO floats, ITPs are equipped with identical SBE-41CP CTD sensors. These low-power instruments (drawing about 


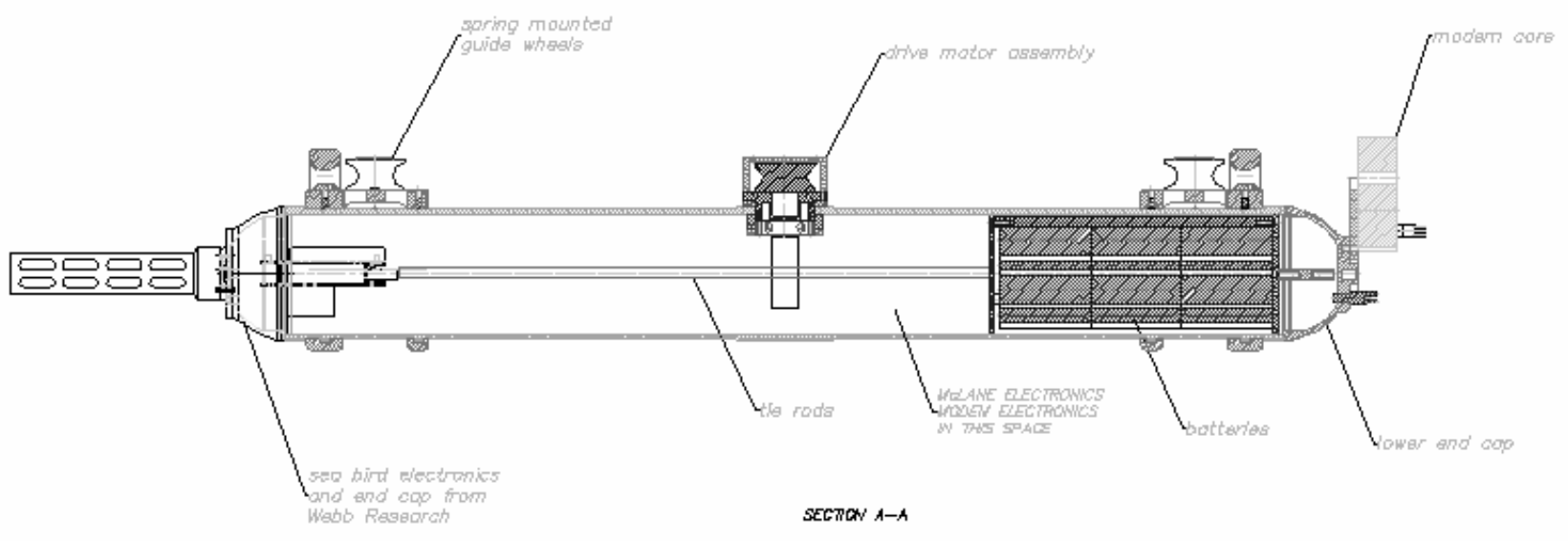

Figure 6: ITP Profiler Unit

$0.25 \mathrm{~W}$ in continuous operation), manufactured by Sea-Bird Electronics, Inc. mount to a hemispherical end cap on the cylindrical pressure case. The ITP samples while profiling in either direction, although data from the down-going profiles is degraded by the wake of the ITP body. Sea-Bird SBE-44 UIMs are integrated to transfer the acquired profile data to the surface controller. With the relatively fast data rate of the Iridium link, we are able send full $1 \mathrm{~Hz}$ sensor sample rate CTD data to shore. High resolution sampling will facilitate study of diffusive layering, intrusions and other finescale structure. Wake errors in down-going profiles largely depend on the strength of the ice-water velocity difference in comparison to the profile speed (dictating the shape of the instrument wake relative to the CTD sensing volume). Data from the first prototype ITPs indicate that there is obvious smoothing of the fine structure in the down profiles versus the up profiles.

In order to facilitate deployment of the devices (Figure 7), the ITP was designed so that it can be deployed through an 11" hole in the ice floe (the maximum diameter that can be bored using conventional hand augers). The ARGO float cylindrical pressure case (6.5" OD) nicely met this requirement. Rather than use the pumped, variable buoyancy system of the float, a traction drive unit (very similar to that used on the Moored Profiler) is mounted roughly midway along the ITP case. (A buoyancy drive like the floats was considered, but a traction drive can develop greater force than the present float-buoyancy unit and thus is better able to function in strong currents with large wire angles.) Torque from a small, brush-type motor running in air within the pressure case is transferred with a magnetic coupler to the drive wheel in the seawater. The wheel is held in tension against the wire by two spring-loaded guide sheaves at the top and bottom of the Profiler. To hold at the rest depth between profiles, the drive motor leads are shorted; the induced EMF associated with motion of the drive wheel serves as a dynamic brake. 
The ITPs are typically ballasted to be neutrally buoyant slightly above their profiling mid-depth. Modest errors in ballasting incur no penalty in performance as the additional energy needed to work against buoyancy in one profile direction is recouped in the other direction. The power requirements are approximately $1 \mathrm{~W}$ to profile at $25 \mathrm{~cm} / \mathrm{s}$.

The profiler controller (PROCON) is designed around the operational MMP system, with the CTD and engineering data passed inductively via the UIM to the surface controller (SURFCON) at the end of each ITP profile. The computer is a Tattletale 8 micrologger from Onset Computer, with $512 \mathrm{Mb}$ flashcard memory.

The standard MMP operating software was modified by McLane for the ITP (software version 3.13 was used in the latest prototype ITPs). The profiler takes profiles based on user programmable sampling depths and schedule, either in regular (evenly spaced) or burst sampling modes. The profiler timing and engineering data (including motor current) are stored in one set of files (Exxxxxx.DAT), while the CTD data are stored in a separate set (Cxxxxxxx.DAT). To prevent the flash card memory root directory from filling up, the software only allows at most the last 40 profiles to be stored on the card -- older files are deleted. The complete raw binary files are sent to SURFCON and subsequently telemetered to a computer at WHOI. Though the present system samples only temperature and conductivity versus pressure, the hardware could support other on-board sensors (such as dissolved oxygen, some bio-optical sensors, and a current meter).

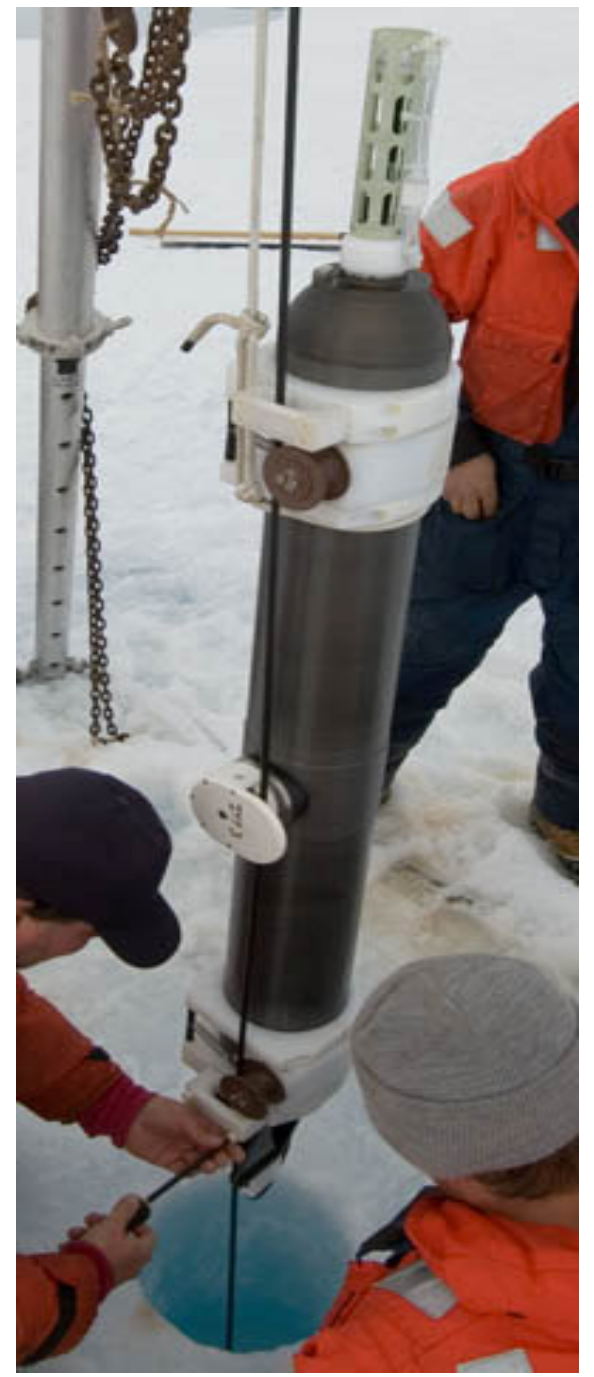

Figure 7: Attaching ITP to mooring wire during deployment.

\section{Tether}

The tether for the ITP (Figure 8) is standard 1/4" plastic-jacketed wire rope with threaded, insulated terminations providing an inexpensive, but durable mechanical and electrical connection. The steel wire acts as the electrical conductor, with a seawater return. The upper 5 $\mathrm{m}$ segment of the tether is encased in urethane, and includes a return wire for the inductive modem circuit that spirals and is encased with the wire rope. At the top end the tether threads 
into a machined fitting which is coupled to the electronics tube and has an electrical breakout connector. The return wire is electrically connected through a similar bulkhead penetrator. At the bottom of the urethane encased section, the return wire is attached to a grounding plate. The termination at the bottom end of the tether acts as the other grounding plate. Shock-absorbing spring bumpers are clamped onto the wire at the top and bottom of the programmed profiling interval to prevent the ITP from impacting the tether terminations. The tether length on the prototypes was $805 \mathrm{~m}$, but shorter lengths could be used in regions of shallow bathymetry (such as the $260 \mathrm{~m}$ Chukchi Cap). Despite the fact that the tether has quite a few turns of the seawater return side of the loop about the jacketed steel center cable, there seems to be no noticable effect on the ability of either SIM or UIM to detect the signal driven by the other over the length of the ITP mooring cable in seawater.

A $250 \mathrm{lb}$ ballast weight (made of $50 \mathrm{lb}$ plates to facilitate transportation) is fixed to the bottom wire termination to add tension to the wire and minimize its catenary. The WHOI CABLE model (Gobat and Grosenbaugh, 2000) was used to determine the attitude of the ITP mooring due to 25,38 , and $51 \mathrm{~cm} / \mathrm{s}$ ice floe drift speeds using several different wire lengths. The model predicts about $5 \mathrm{~m}$ of vertical uplift of the bottom termination at $25 \mathrm{~cm} / \mathrm{s}$, about $32 \mathrm{~m}$ at 38 $\mathrm{cm} / \mathrm{s}$ and about $85 \mathrm{~m}$ at $51 \mathrm{~cm} / \mathrm{s}$. Horizontal displacement of the bottom termination is indicated to be about $100 \mathrm{~m}, 220 \mathrm{~m}$, and $350 \mathrm{~m}$, respectively. To accommodate the catenary of the ITP mooring at times of ice floe drift speeds up to $35 \mathrm{~cm} / \mathrm{s}, 25$ or $30 \mathrm{~m}$ of extra cable (beyond the programmed maximum profiling depth) is needed. So mooring cables of about $790 \mathrm{~m}$ length should allow for profiles as deep as $760 \mathrm{~m}$ in the vast majority of ice drift conditions. At the extreme instances when drift rates approach $51 \mathrm{~cm} / \mathrm{s}$ drift, it may be impossible for the profiler to climb the wire against the current, making the maximum depth of the tether moot.
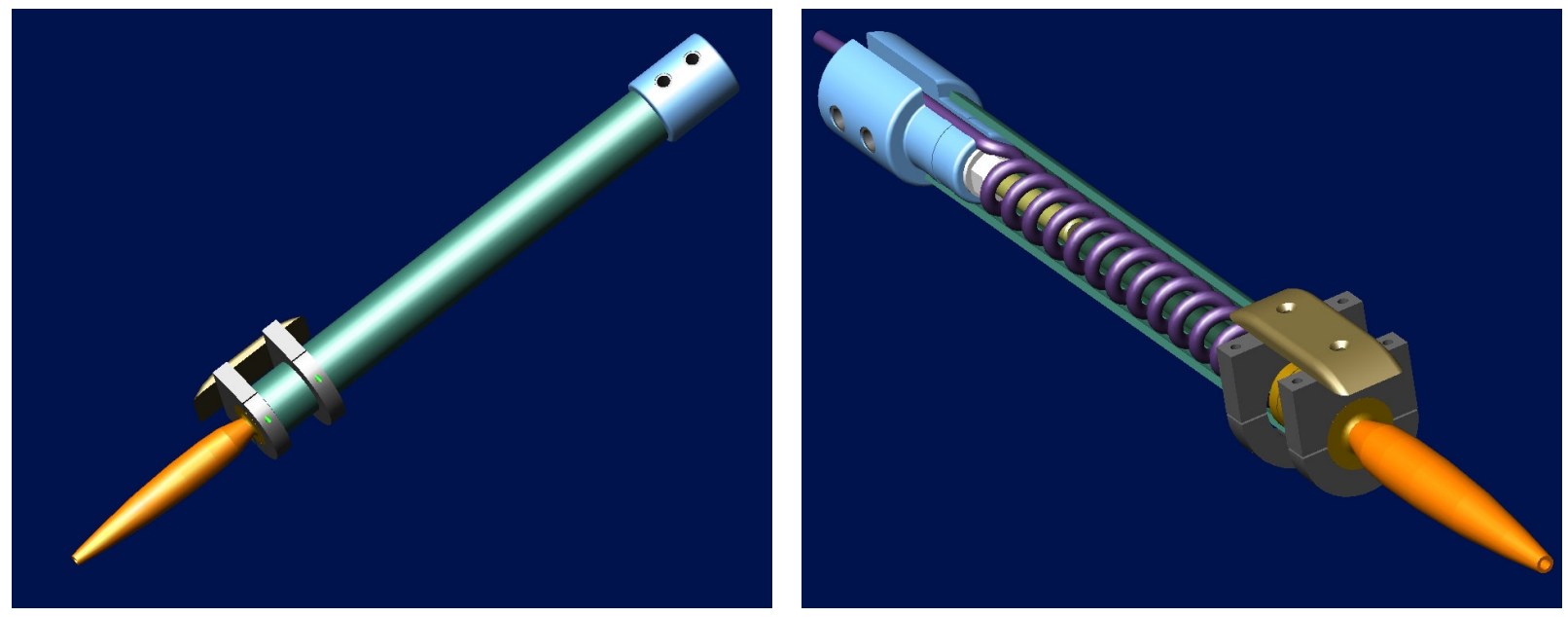

Figure 8: ITP Cable Assembly, upper 5 m of 805 m segment: external (left), internal (right). 


\section{Power}

Based on operation of the prototype ITPs in the field, battery packs totaling 36 lithium BCX "DD" batteries should be capable of powering the surface electronics for over 4 years at 2 profiles per day (after derating the batteries to account for cold temperature degradation), while 24 cells should sustain the underwater profiler for over 2.5 years.

Individual battery slices (each nominally $1250 \mathrm{Wh}$ ) supply power to the surface electronics, with connectors on the SURFCON board accommodating as many as six packs (but the present tube length of the surface buoy limits space to 4 battery slices at once). A 12-cell $10.8 \mathrm{~V}$ battery slice consists of three cells wired in series with a diode and four series wired in parallel. A 3 A fuse also guards against accidental short circuiting. Together, three packs should produce at least $3300 \mathrm{Wh}$ after derating for temperature. Based on a data throughput of 100 kbytes/day from the profiler ( 2 profiles), 100 bytes/s inductive modem link, and 200 bytes/s Iridium link, the power requirements are estimated to be less than $2 \mathrm{Wh} / \mathrm{day}$.

The profiler has its own battery pack consisting of 24 lithium "DD" cells totaling 2500 Wh and sufficient for an estimated system endurance of 1.5 million meters of profiling (derated for temperature) based on a total energy consumption rate of $1.35 \mathrm{~W}$ during profiling operations (= CTD + motor + UIM transfer + overhead $)$. Consequently, with a standard battery pack, ITPs scheduled to profile once per day (either up or down) are expected to have sufficient power for at least 4.5 years, twice per day (one each way) for 2.5 years, and 4 profiles per day ( 2 up, 2 down) for 1.25 years. Furthermore, there is sufficient room in the profiler tube for an additional 1250 W-hr battery which would increase the lifetime by $50 \%$.

\section{SOFTWARE OPERATION}

\section{SURFCON}

The data controller in the surface package (SURFCON) obtains technical status information, locations from GPS, operates the surface inductive modem (SIM) interface, communicates with the underwater inductive modem (UIM) on the profiler, obtains the data from the instrument (PROCON), and feeds the information to the Iridium satellite unit (ISU). Hardware wiring diagrams are given in Appendix A, and the operating instructions summarized here are detailed in Appendix C.

There are two programs that are used for ITP testing and deployment: "xbt" is a diagnostic program for testing the individual components prior to deployment, and "surfcon" is the acquisition program. 
The program "xbt" exercises different components of the system including: writing files to flash memory; reading voltages from the ADC; turning on each device for serial communications; the suspend function; placing a call with the ISU and sending a test message; displaying the GPS RMC string, date and time; setting the real-time clock (RTC); and powering SIM and UIM, and reading status from UIM.

The "surfcon" software is the automated program for operating the surface package during a deployment with a profiler to obtain and telemeter the data back to the laboratory (Figure 9). In order to ensure reliability, the operating software is written to accommodate all known states, but a hardware watchdog timer is included to increase the likelihood of recovery from stalled states or a genuine processor problem. On powerup (or reset) the Persistor CF-2 controller begins running the ITP operation program (SURFCON.PXE) indicated in its AUTOEXEC.BAT file. After initializing the hardware, loading the operating variables (by reading the configuration file ITPSYS), and opening a logfile to record operating events, the program enters the main program loop which responds to interrupts from the tone detector to receive data from the profiler, and keeps two separate schedules: one for obtaining engineering status (and GPS location) information, and one for transferring the queued files via Iridium.

The main loop begins by determining the next status and transmission times, updating and saving the status and location file at the first occurrence of a new day, then reverts the SURFCON controller to suspend mode until the earlier of the status or transmission times is encountered. Suspend mode is essentially a power-off condition whereby the realtime clock maintains time and main memory while drawing only $10 \mu \mathrm{A}$. SURFCON will awaken from the suspended condition for two reasons: 1) wake on time, when either the next status check or time to conduct ISU operations has been reached, or 2) wake due to tone detect, from the UIM or RESET switch.

When the time for a status update (including GPS location) is reached, then SURFCON saves the buoy temperature and voltages information in the status and log files and begins QCHECK. When the time for an ISU transmission is reached, then SURFCON begins QCHECK. When the tone detector signals, SURFCON awakens and enters a loop to conduct communications between the SIM in the surface package and the UIM in the profiler instrument. The complete IM protocol is described in Appendix G. Briefly, SURFCON prompts with REQNEW and PROCON responds with the name of each new profiler file to be relayed, the file, and checksum info. This process repeats until all the new files are transferred to SURFCON, which stores the files in subdirectories by year and adds the filenames to the queue for transmission. Upon completing the SIM/UIM communications, SURFCON begins the QCHECK routine. 
Scheduled Iridium uploads occur during QCHECK, where SURFCON reads filenames from the queue, powers and registers the ISU, dials the WHOI data serving computer, sends all files in the queue, then terminates the call. The SURFCON-to-logger computer communications, including provisions to allow the logger to pass some simple commands to SURFCON via the Iridium link, are described in Appendix H. At the present time, the commands are limited to specific file requests and directory listings from both SURFCON and PROCON. Upon completing QCHECK, SURFCON operates on any received command files, and then returns to the start of the main loop.

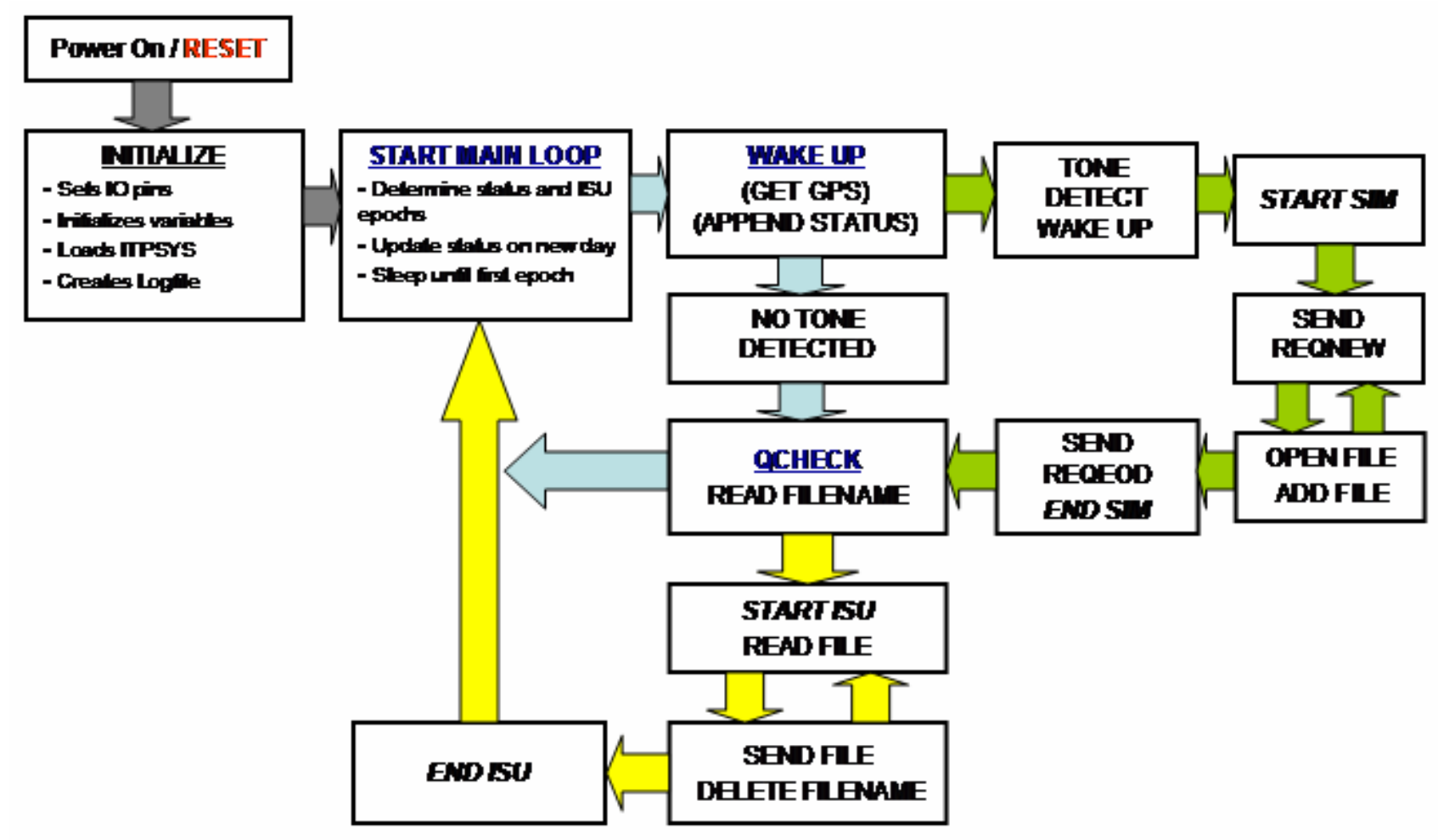

Figure 9. SURFCON operation block diagram

The only way to break out of SURFCON externally is by activating the RESET line via the console port (or with a push button switch on the SURFCON board). In addition, there is a watchdog timer that will cause the PF- 2 operating system to restart after 20 minutes of inactivity, to protect against software code failure. After resetting the operating software, the user has 5 seconds to break out the SURFCON program by entering CTRL-X twice. Resets are incremented and recorded in the status files, and a new log file is opened. 


\section{PROCON}

The electronic hardware and operating program of the profiler controller, or PROCON, is based on the McLane Moored Profiler (MMP) Board Stack with a Sea-Bird Underwater Inductive Modem (SBE-44) OEM Stack. PROCON controls the drive motor, operates the CTD, records the files on a flash card, and communicates the data to SURFCON via the inductive modem link. The ITP profiler software allows for either all, or a limited revolving number of profiles to be saved on the flash memory card. A limitation of the file allocation in the prototype PROCON software dictated that a maximum of about 2045 profiles ( 2 files per profile) could be saved in the root directory. The battery capacity of the profiler with a standard pack is expected to be between sufficient for 1600 to 2000 (750 m long) profiles, so it is unlikely that this software limitation will be reached. However, since ITPs are not expected to be recovered, PROCON is usually configured for file deletion after each profile (keeping only the prior 40 files). A detailed description of the PROCON operating instructions is provided in Appendix F.

At the end of each profile, PROCON downloads data from the CTD into one file, formats engineering information into a second file, and relays both via the inductive modem to the surface unit (SURCON). These data files are queued along with the SURFCON status files and relayed to shore.

\section{TESTING}

A series of bench tests, environmental tests, tank tests, and dock tests were performed on each prototype ITP prior to deployment to ensure full functionality in the field (Figure 10).

Bench tests are used to qualify individual components. Environmental tests to the electronics in the surface packages of the prototypes indicated reliable operation and data transmission at temperatures as cold as $-38^{\circ} \mathrm{C}$. Profilers were exercised and wet weighed (for ballasting) in the McLane Research 15-m-deep freshwater tank. Finally, each ITP surface unit and matching profiler were operated together while suspended from the WHOI pier using a 15-m long test mooring cable.

It became apparent during the testing of ITP1 that the GPS locations contained spikes at regular intervals while the antenna was fixed to single location. Due to the location spikes and cable loss along approximately 40' of low-loss coaxial cable between the transceivers located in the environmental chamber and the antennae mounted outside, mean location errors were over $100 \mathrm{~m}$. Installed in the ITP surface packages, field tests documented occasional location deviations up to $60 \mathrm{~m}$. This noise was more apparent when voltage decreased as battery packs were exhausted. These spikes are relatively easy to identify and edit from the recovered GPS 
time series. Only a few large position spikes were documented while testing ITP3 (Figure 10), but the field data also exhibit occasional position errors. In the next generation ITPs, the GPS sensor is separate from the Iridium transmitter and includes a backup battery to save the last location and reduce location errors.
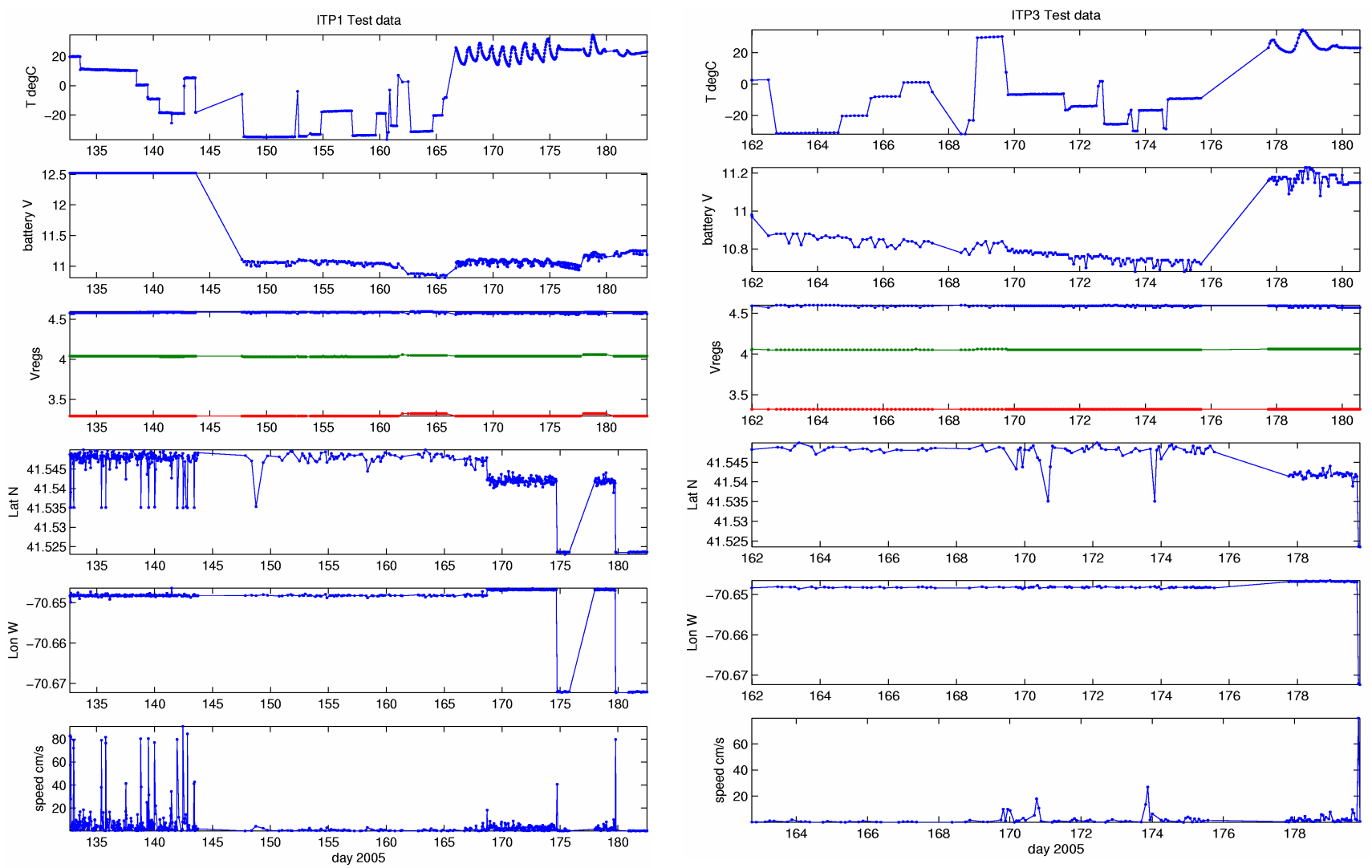

Figure 10: Temperature, voltage, and locations from ITP1 (left) and ITP3 (right) cold, dock, and field tests.

\section{CTD Sensor Care}

The first three ITPs incorporated Sea-Bird Electronics 41-CP CTD sensors. Calibrations are performed by Sea-Bird prior to installation on the ITP, and calibration coefficients are stored in non-volatile memory. Instructions on how to configure the CTD sensors for PROCON are given in Appendix E.

To avoid altering the calibration, it is extremely important to keep the conductivity cell clean if the sensors are immersed during testing. Users will typically fill the CTD plumbing with ultra-clean DI water, taking care to get air bubbles out, and then cap the intake and exhaust ports 
or loop a tygon tube from the TC duct intake to the exhaust barb. Care must be exercised during shipping and deployment that this water does not freeze and damage the sensors. If the cell has been fouled, one can attempt to clean it by washing/rinsing the cell with warm triton solution or running the warm solution in a closed loop with the CTD pump for half an hour. In either case,a laboratory conductivity calibration will be required to achieve 0.002 pss accuracy in derived salinity.

To prevent wear to the pump, it should not be powered when testing the profiler in air. The pump power feed from PROCON (J7) pin 6 to the 41CP 10-pin header connector (JP-1) pin 10 may be temporarily removed if a deployment is being simulated.

\section{Profiler Ballasting}

For optimal performance, the profilers should be ballasted to be neutrally buoyant near the mid-depth of the planned profiling interval. Ballasting will, in turn, depend on the seawater conditions at the deployment site (which must be estimated before deployment) and on the in situ volume (V) of the instrument. The ITP profiler body is nearly neutrally buoyant in freshwater at laboratory temperature. For typical polar ocean applications (where the seawater is denser), additional mass will be required.

Instrument ballasting is an application of Archimedes principle: buoyancy force equals the weight of the displaced water, $\rho \mathrm{Vg}=\mathrm{m} \mathrm{g}$, where $\mathrm{m}$ is the instrument mass (weighed in air), $\mathrm{g}$ is gravitational acceleration, and $\rho$ is the in situ seawater density. The difficult part is estimating the change in $\mathrm{V}$ for the different pressure and temperature conditions on site. $\mathrm{V}_{\text {bath }}$ is estimated from the air weight, water weight and the density of the bath water. For a given estimated ocean in situ density $\left(\rho_{\mathrm{i}}\right)$, the change in mass is related to $\rho_{\mathrm{i}}$ multiplied by $\mathrm{V}_{\mathrm{i}}$, where $\mathrm{V}_{\mathrm{i}}$ is the in situ volume. Based on the properties of the materials in the instrument, temperature $(\alpha)$ and compressibility $(\beta)$ adjustment terms are estimated in:

$$
\mathrm{V}_{\mathrm{i}}=\mathrm{V}_{\text {bath }} *\left[1+\alpha *\left(\mathrm{~T}_{\mathrm{i}}-\mathrm{T}_{\text {bath }}\right)-\beta *\left(\rho_{\mathrm{i}}-\rho_{\text {bath }}\right)\right]
$$

Normally, $\alpha$ and $\beta$ will be positive (instrument volume contracts under pressure and at colder temperatures). For the prototype ITPs, $\alpha$ was estimated to be $3.25 \mathrm{cc} /{ }^{\circ} \mathrm{C}$ and $\beta$ was estimated to be $0.1 \mathrm{cc} / \mathrm{db}$. Operation of the prototypes in the field verified the selection of these estimates. Finally, the amount of ballast to be added to an ITP for a given ocean temperature and density is the difference of the air weight minus $\rho_{\mathrm{i}} * \mathrm{~V}_{\mathrm{i}}$.

To determine the expected seawater conditions, salinity and temperature profiles from the planned deployment region from previous hydrographic surveys or climatological databases need to be obtained. The ranges and midpoints of temperature and density need to be calculated over 
the vertical depth that the profiler will operate. Based on this information a mean seawater density is selected, and the water weight of the profiler may be adjusted accordingly.

To determine the water weight of the profiler, the instrument (complete with CTD sensors and battery packs) must be lowered into a freshwater tank and weighed to at least 1 gram accuracy. Since the instrument without ballast is positively buoyant, this entails adding a small known ballast weight (typically less than $5 \mathrm{~kg}$ of lead weights) to the instrument. For a stable water weight determination, the temperature of the instrument must be allowed to equilibrate with the temperature of the bath. Space inside the battery compartment and bottom endcap is provided for adding lead ballast. When the ballast is stored inside the profiler pressure vessel, as it typically is, then the weight of the lead ballast that goes into the calculations is the same as the air weight.

\section{IMPLEMENTATION}

\section{Field Installation}

The ITP system is designed so that it may be deployed directly from an icebreaker using man hauling or helicopter ferrying, or up to 300 hundred miles from fuel depots using a single small aircraft (Figure 11). This was achieved by minimizing the size and weight of the system and the deployment gear. The ITP surface buoy weighs $70 \mathrm{~kg}(150 \mathrm{lb})$, the profiler weighs $30 \mathrm{~kg}$ (70 lb), and a $805 \mathrm{~m}$ wire tether on an aluminum spool is $255 \mathrm{~kg}$ (560 lb). Altogether with the tether ballast, the ITP hardware totals approximately $450 \mathrm{~kg}(1000 \mathrm{lb})$. Deployment technicians, emergency survival gear, and the minimum deployment equipment bring the payload up to 1000 $\mathrm{kg}(2200 \mathrm{lb})$. This weight may be transported up to a maximum 280 miles roundtrip by a medium lift helicopter (such as the Bell 212), or over 600 miles roundtrip by the DeHavilland Twin Otter airplane. Of course, the amount of equipment that can be transported during any single flight is a function of the distance of the site from the ship. If the roundtrip distance is reduced, several ITP systems could be loaded onto and deployed from a single Twin Otter.

The ITP deployment operations conducted to date have been conducted as part of the Beaufort Gyre Exploration Program (BGEP; http://www.whoi.edu/ beaufortgyre) during Joint Western Arctic Climate Studies (JWACS) cruises, and supported by the Canadian Coast Guard icebreaker Louis S. St. Laurent utilizing BO-105-CB helicopters for reconnaissance to select an appropriate icefloe, and to transport gear and people for the deployment. These particular helicopters can carry up to 4 passengers and have a stated payload (fuel and cargo) of approximately $675 \mathrm{~kg}$ (1500 lb), so several flights were needed to transport a single ITP system, deployment apparatus, and personnel. 
Reconnaissance flights to select the icefloe include 1-2 scientists (in addition to pilot and rifle bearer) with a 2" hand auger and measuring tape. Multiyear icefloes are identified, landed upon and drilled to determine ice thickness. The ideal platform for an ITP deployment is a multiyear ice floe (which can be identified by the lighter shade of blue color in melt ponds in summer) that is relatively thick ( 2.5 to $4.5 \mathrm{~m})$, level, and sparsely ponded.

For the deployment operation, 2-3 technicians (plus rifle bearer) and as much as $1100 \mathrm{~kg}(2400 \mathrm{lb})$ of gear must be transported to the selected site. Using helicopters or by man hauling, each ITP system can be transported to the deployment site in several pieces: surface package, wire tether on aluminum spool, anchor weight, and the Profiler unit. Typically 4-5 slingloads (or sled loads) of gear only are transported to the site, then another 1-2 flights transport personnel and equipment stored in the cargo bay.

With the aid of a light weight, portable tripod, the tether ballast weight and subsurface tether is deployed through a 10.5-11" diameter hole augered through the icefloe (Figure 12). At a convenient point, the ITP is mounted on the wire (two bracket connections) and eased down the wire through the ice hole. After making one mechanical and electrical connection at the surface unit, the package is positioned onto a wooden palett over the hole and the tripod is disassembled. Based on the preprogrammed sampling plan entered in the ITP profiler, the instrument will then start working.
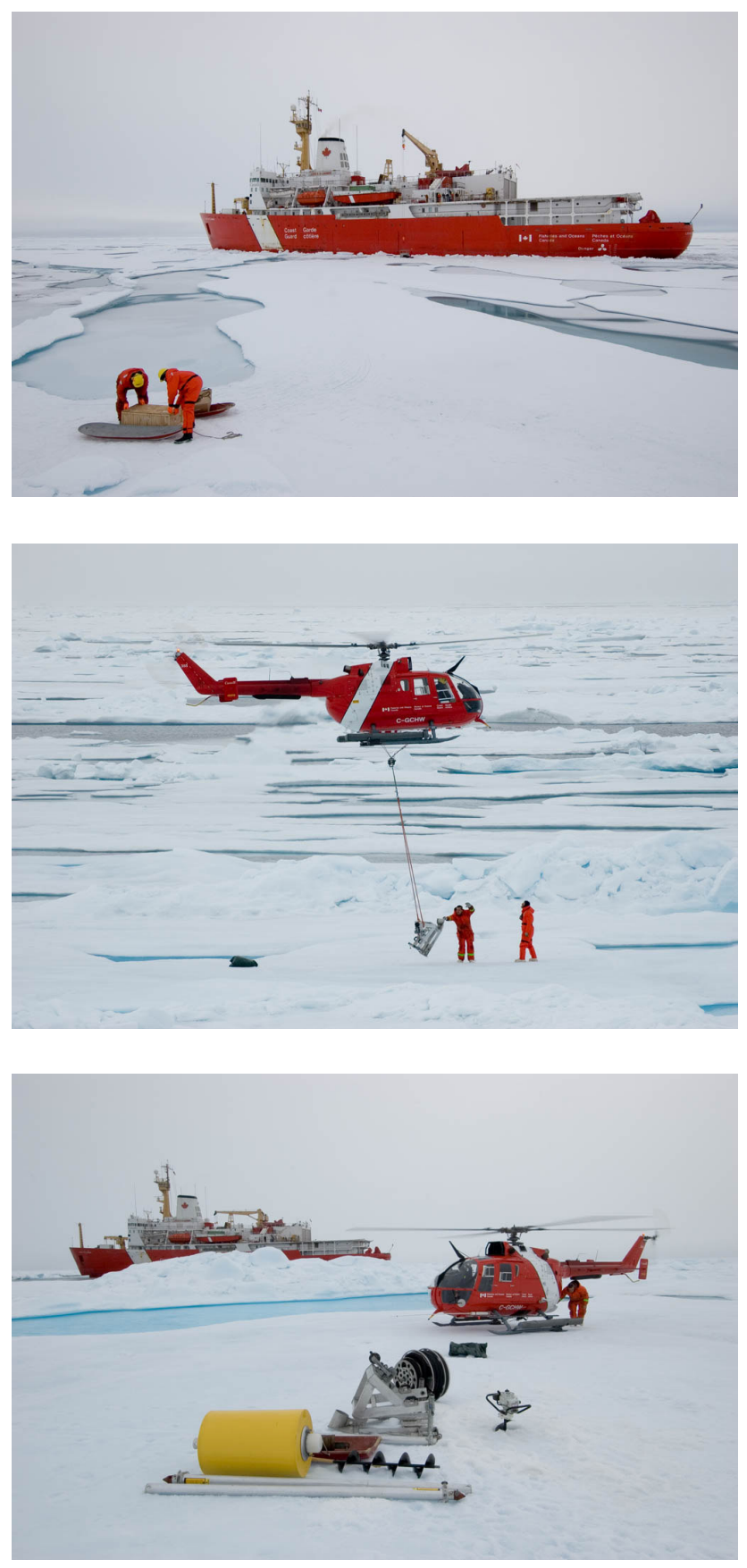

Figure 11: Transporting ITP gear to deployment sites using hand sleds and helicopters (in slings and cargo). 
The operations to deploy the prototype ITPs each took between 2-4 hours, with 3-4 helicopter flights (sled loads) to return the deployment gear and personnel to the ship. From first landing to last departure, the full ITP system can be deployed in 4-8 hours.
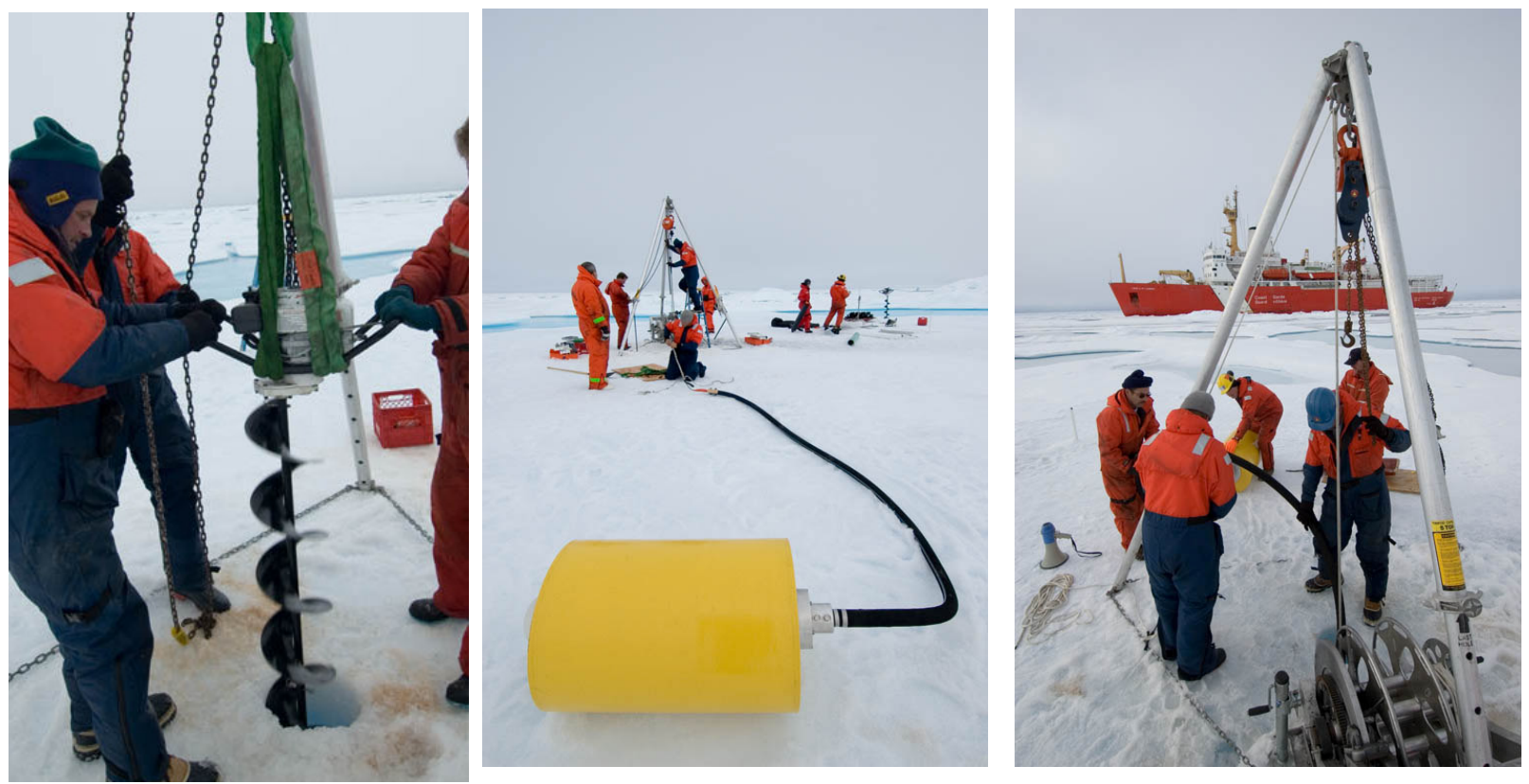

Figure 12: Augering 11" deployment hole with tripod assistance (left). Surface package after attachment to ice-strengthened cable (center). Lowering surface package and cable through ice hole with slip line (right).

\section{Prototypes}

One ITP (serial number 2) was deployed in 2004 and two were deployed in 2005 (serial numbers 1 and 3) from JWACS cruises on the CCGS Louis S. St. Laurent (Figure 13). Both ITP 1 and 2 utilized helicopter transport, while ITP 3 was deployed by lowering equipment over the side of the icebreaker and man hauling it into position. All three ITPs were deployed with IMB buoys several meters away and installed where the ice was expected to drift through the BGEP bottom-tethered mooring array over the following year. Developed and built at the US Army CRREL, the IMB primarily acquires time series of ice temperature profiles and measures the growth and ablation of sea ice (and snow) at the top and bottom ice surfaces. The combination of the ITP systems and IMB systems are an example of an Ice-Based Observatory (IBO) that obtains cotemporaneous sea ice and seawater properties for a sustained duration.

ITP \#2 was deployed in the Beaufort Sea on August 19, 2004 at $77.174^{\circ} \mathrm{N}, 141.238^{\circ} \mathrm{W}$ during the JWACS 2004 cruise. ITP 2 together with IMB (07413) buoy were deployed on a $4 \mathrm{~m}$ thick multiyear ice floe. ITP 2 collected 244 profiles on a fast sample schedule of 6 one-way profiles between 10 and $750 \mathrm{~m}$ depth each day. 
ITP \#1 was deployed in the Beaufort Sea on August 15, 2005 at $78^{\circ} 51.1^{\prime}$ N, $150^{\circ} 15.9^{\prime}$ W as part of the JWACS 2005 cruise. ITP 1 and IMB (07949) were deployed on a $4.6 \mathrm{~m}$ thick multiyear ice floe.

ITP \#3 was deployed in the Beaufort Sea on August 23, 2005 at $77^{\circ} 36.1^{\prime} \mathrm{N}, 142^{\circ} 11.8^{\prime}$ W (about $200 \mathrm{~km}$ to west of ITP1), also part of the JWACS 2005 cruise. ITP 3 and IMB (07950) were deployed on a $3.5 \mathrm{~m}$ thick multiyear ice floe.

Both ITP1 and ITP 3 are operating on a fast sample schedule of 4 one-way profiles between 10 and $760 \mathrm{~m}$ depth each day.

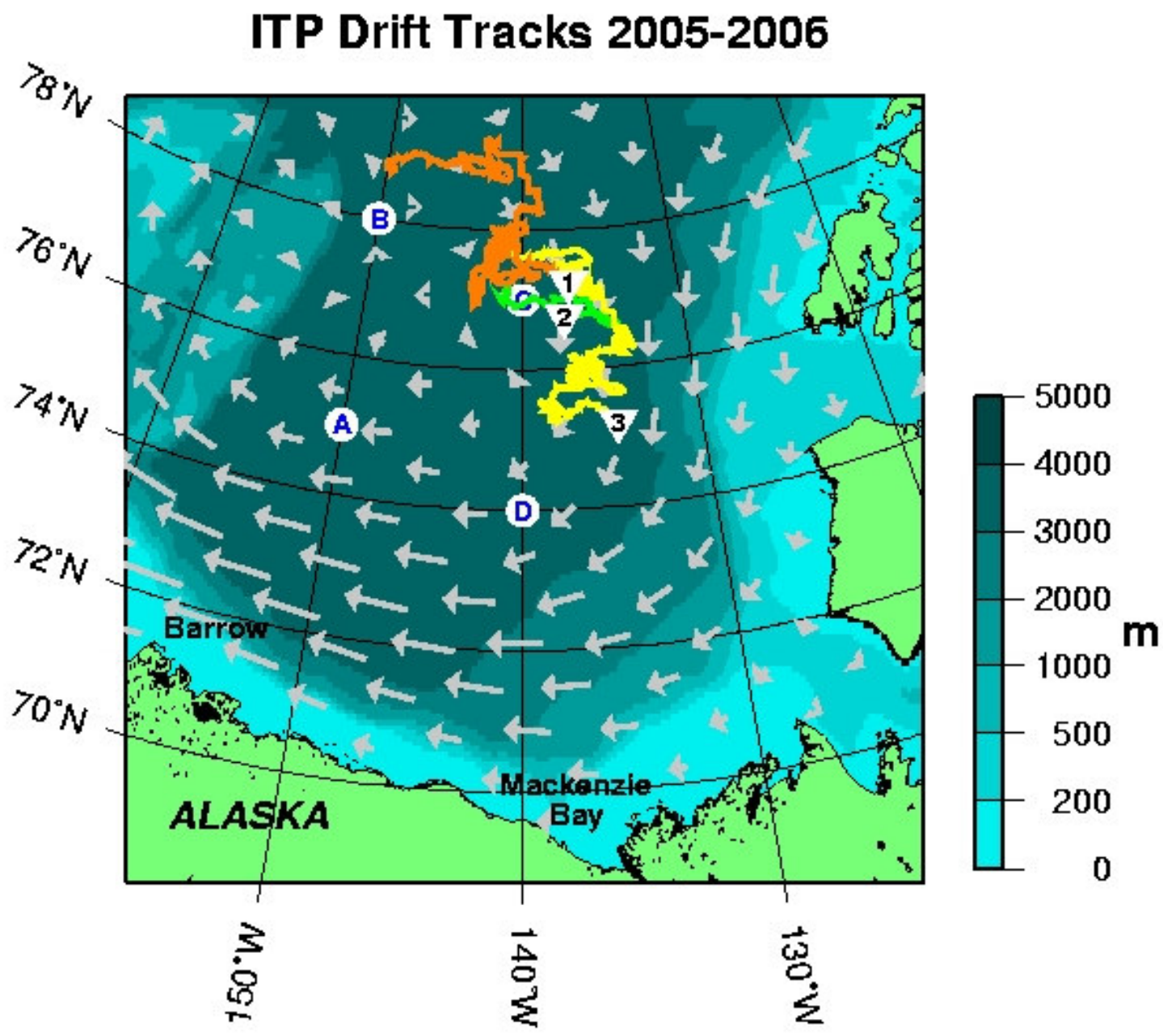

Figure 13: ITP prototype drift tracks (as of June 2006): ITP2 in 2004 (green), ITP1 (orange) and ITP3 (yellow) in 2005. Numbers mark most recent locations. Letters indicate locations of BGEP moorings. Climatological ice drift vectors from the IABP are grey vectors superimposed on shaded bathymetry. 


\section{DATA DISTRIBUTION}

\section{Iridium Upload}

Each ITP is expected to return 1600 or more daily high-vertical-resolution measurements of upper Arctic Ocean temperature and salinity in near real time spanning all seasons over a three-year lifetime. To receive all the data uploads, two logger computers running Linux are connected by a Rev 2 Hayes modem to dedicated telephone lines at WHOI (Appendix H). The logger computers are configured for binary data acquisition and can handle partial file transfers (due to Iridium link loss) by instructing SURFCON to retransmit incomplete files starting after the last correct packet received by the logger. This entails switching the master-slave responsibilities in the protocol such that once a call connects, SURFCON prompts the logger. The expected response is a request to either restart a failed transmission, transmit a new file (most often the case), or receive a command packet.

SURFCON normally sends 2 files per profile (about 20 kbytes and 30 kbytes, respectively), as well as a daily ( 4 kbyte) status file at the first transmission epoch after 0000Z. When SURFCON sends a file via Iridium to the logger computer at WHOI, it is done in 2048 byte packets, each with a similar header. If a packet is received correctly, the logger strips off the header bytes and appends the current file, so there is no re-assembly necessary once data or engineering files arrive, and standard MMP data extraction programs may be used to access the data (Appendix H).

Data (Cxxxxxxx.DAT) and engineering (Exxxxxxx.DAT) files from SURFCON are sent to the logger preceded with a 50 character PROCON metastructure and appended with a 70 character SURFCON metastructure. Note that the 2 structures are identical for the first 49 characters in order to assure that the description source of each file is embedded in that file and likely recoverable from at least one place or the other.

The status file is of the name type mmddyyyy.STA, where: $\mathrm{mm}=\mathrm{month}, \mathrm{dd}=\mathrm{day}$ and yyyy=year. A status file is typically a sequence of entries of the form:

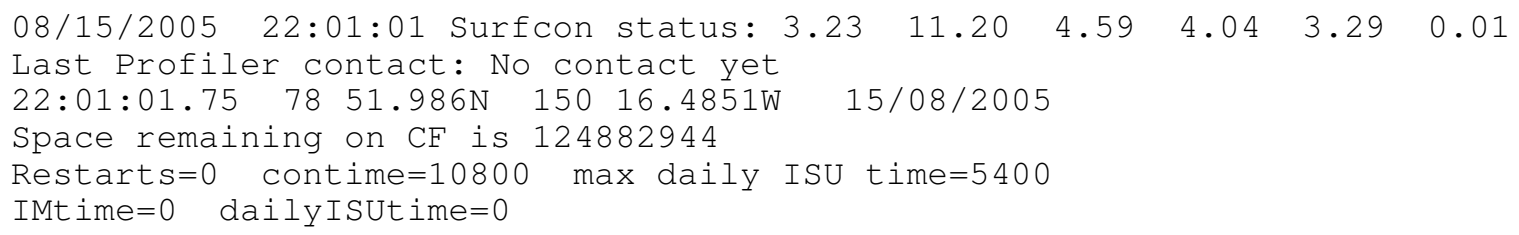

where the first date and time represent when the SURFCON awoke at the specified interval to assemble the status information; the 6 floating point numbers are the Celsius temperature inside the controller housing, the lightly loaded SURFCON battery voltage, the $4.5 \mathrm{~V} \mathrm{DC} / \mathrm{DC}$ converter 
voltage for the ISU, the 4.0V DC/DC converter voltage for the SURFCON board power, the output of the Persistor 3.3V regulator and the unused auxiliary 3.3V regulator. On the next line, there is normally a date and time specifying the last time the PROCON contacted the SURFCON for a data transaction. The third line is the time, latitude, longitude and date from the GPS receiver. The GPS time, if acquired, is used to reset the Persistor realtime clock. The remaining lines indicate the space (in bytes) remaining on the SURFCON flash card, and other operational information. The last line in the status file includes the number of IM wakeups and the time in seconds that the Iridium link has been in use during that day.

SURFCON also supports commands to allow access to specific data files both on its own flash card and on PROCON, as well as directory lists on each, SURFCON status and profiler UIM status. Consequently, the logger is also capable of handling outbound traffic, i.e. a command packet, from logger to SURFCON (Appendix H).

\section{Preliminary Processing}

All of the telemetered data are processed and made publicly available on the ITP internet web site (http://www.whoi.edu/itp/data) as soon as they are received. Within a couple of hours after arriving on the WHOI logger computer, the files from each profile are accessed by a separate computer that acquires the binary data files from the logger computer, extracts and despikes the data, averages profiles into convenient 1 or $2 \mathrm{~m}$ bins, produces plots and saves the data in ASCII and MATLAB format files.

The raw GPS buoy location data are also made available on the ITP web site in an ASCII file: itpNrawlocs.dat. On ITP 1 and ITP 3 ITP, GPS fixes were scheduled every hour, but are sometimes missed if the acquisition time exceeds 2 minutes. The raw locations provided on the web site are currently not screened to remove spurious glitches, but the location time series are filtered and interpolated to produce locations for each profile data file.

Depth-bin-averaged profiler data files (with interpolated location) are available in two formats: itp $N$ grddata.tar.Z or itp $N$ grddata.zip, where $N$ is the ITP number. Each profile is named itpNgrdnnnn.dat, where $n n n n$ is the profile number. Each file has one line of header data: "\%year day longitude $(\mathrm{E}+)$ latitude $(\mathrm{N}+)$ ndepths", the next line contains the values, the third line is a header for the profile data: "\%year day pressure(dbar) temperature(C) salinity", and the remaining lines are the data, except for the last line which is: "endofdat". The convenient gridded data are for general use, but the raw data files are also available by ftp. 


\section{Sample data from ITP2}

ITP 2 was programmed with an accelerated sampling plan of 6 one-way profiles per day between 10 and $750 \mathrm{~m}$ depth. Profiling speeds of $25-28 \mathrm{~cm} / \mathrm{s}$ were achieved with profileaveraged motor current from the 10-11 V battery supply ranging between 120 and $260 \mathrm{~mA}$ (averaging $140 \mathrm{~mA}$ ). Larger motor currents were observed at times of fast ice floe motion (exceeding $25 \mathrm{~cm} / \mathrm{s}$ on occasion) when we presume that drag on the Profiler and wire are increased (Figure 14).
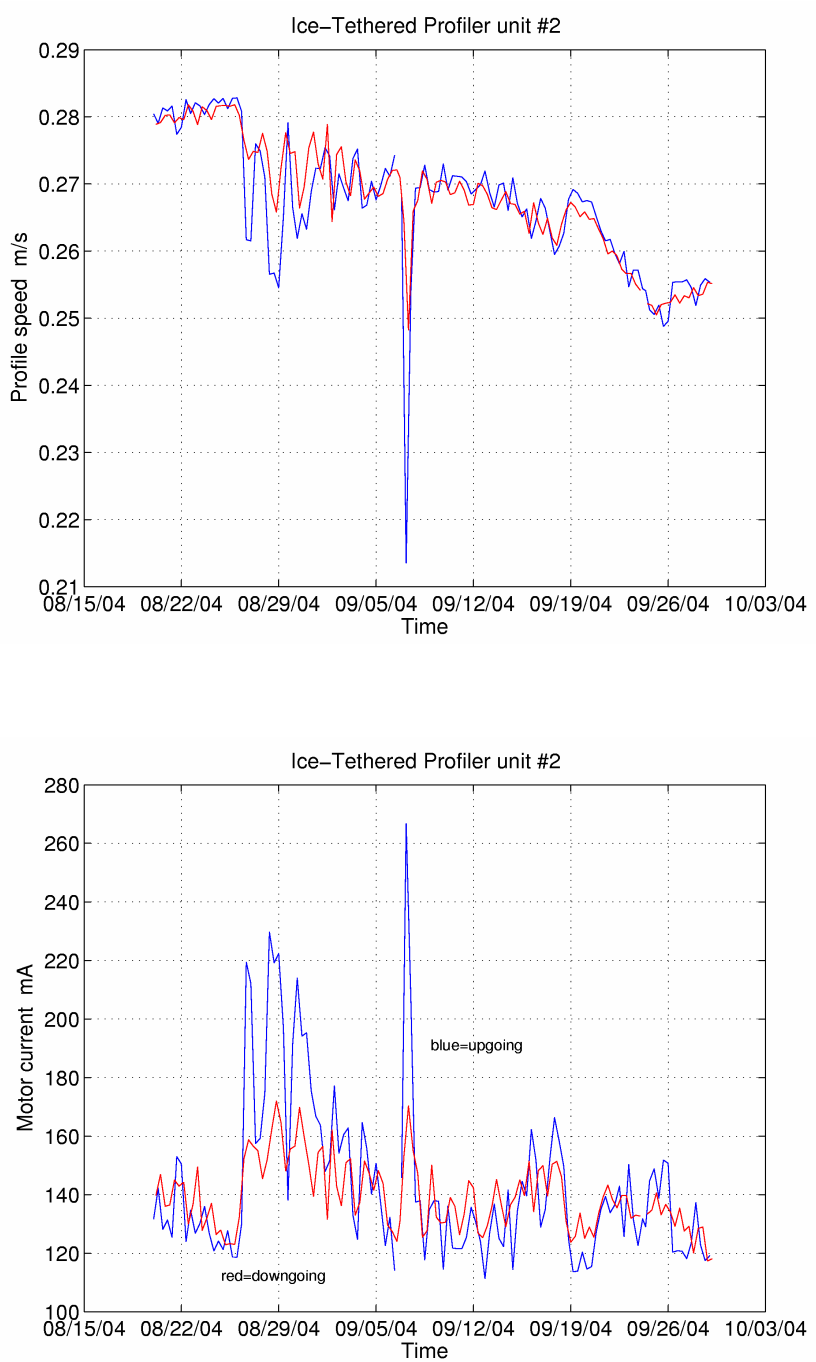

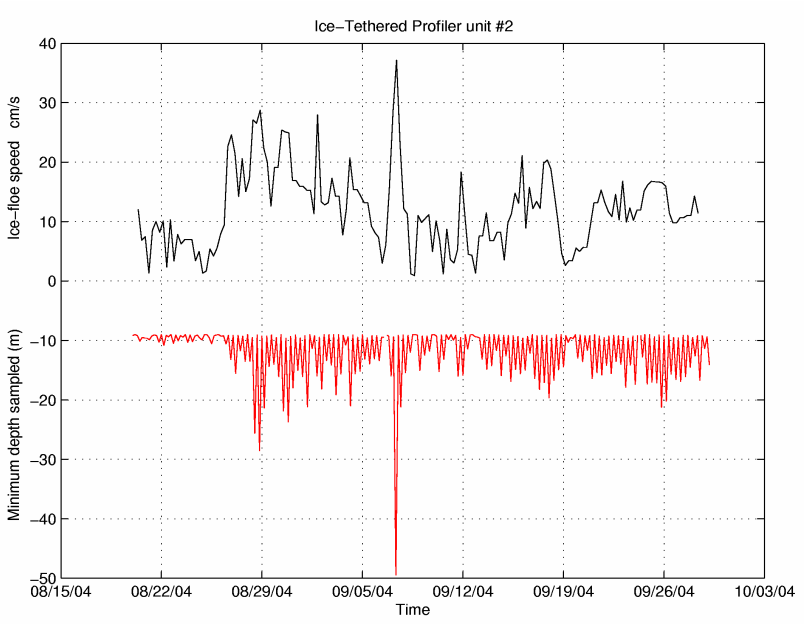

Figure 14. (Top) Profile-averaged motor current versus time. Upgoing profile data are shown in blue, downgoing in red. On average, the upgoing and downgoing motor currents are comparable, indicating that the Profiler was ballasted neutral near the midpoint of the sampled depth interval. Larger motor currents, particularly on upgoing profiles, are seen at times of large ice floe translation. (Middle) Average profile speed versus time. Upgoing profile data are shown in blue, downgoing in red. (Bottom) Time series of ice floe speed (black curve) and minimum pressure sampled on each ITP profile (red curve). While the up-going profiles always reached the shallow programmed stop depth of $10 \mathrm{~m}$, during times of fast ice floe motion the Profiler tended to drift down from its resting depth between cycles due to drag from the relative flow past the instrument. 

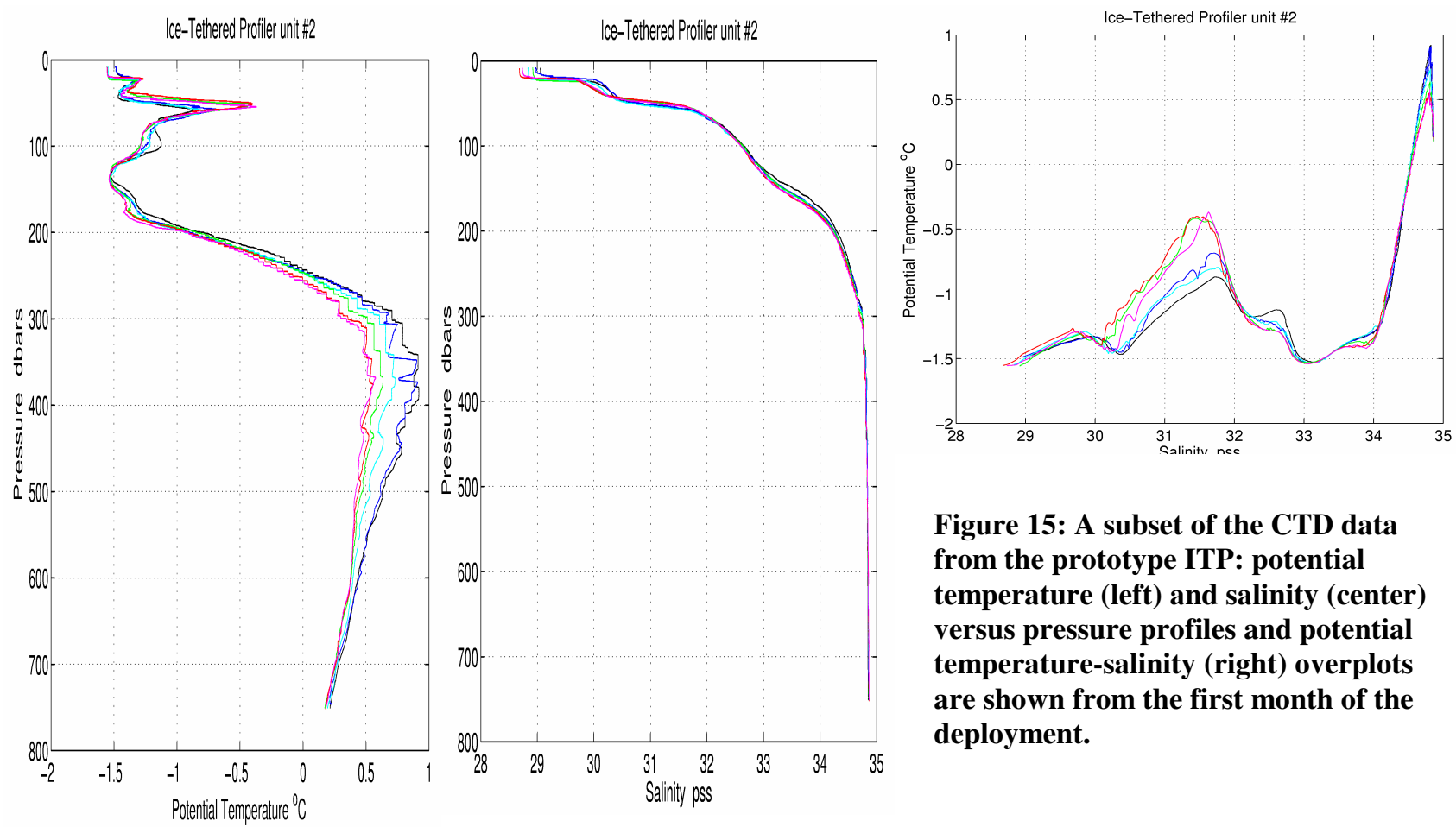

Figure 15: A subset of the CTD data from the prototype ITP: potential temperature (left) and salinity (center) versus pressure profiles and potential temperature-salinity (right) overplots are shown from the first month of the deployment.
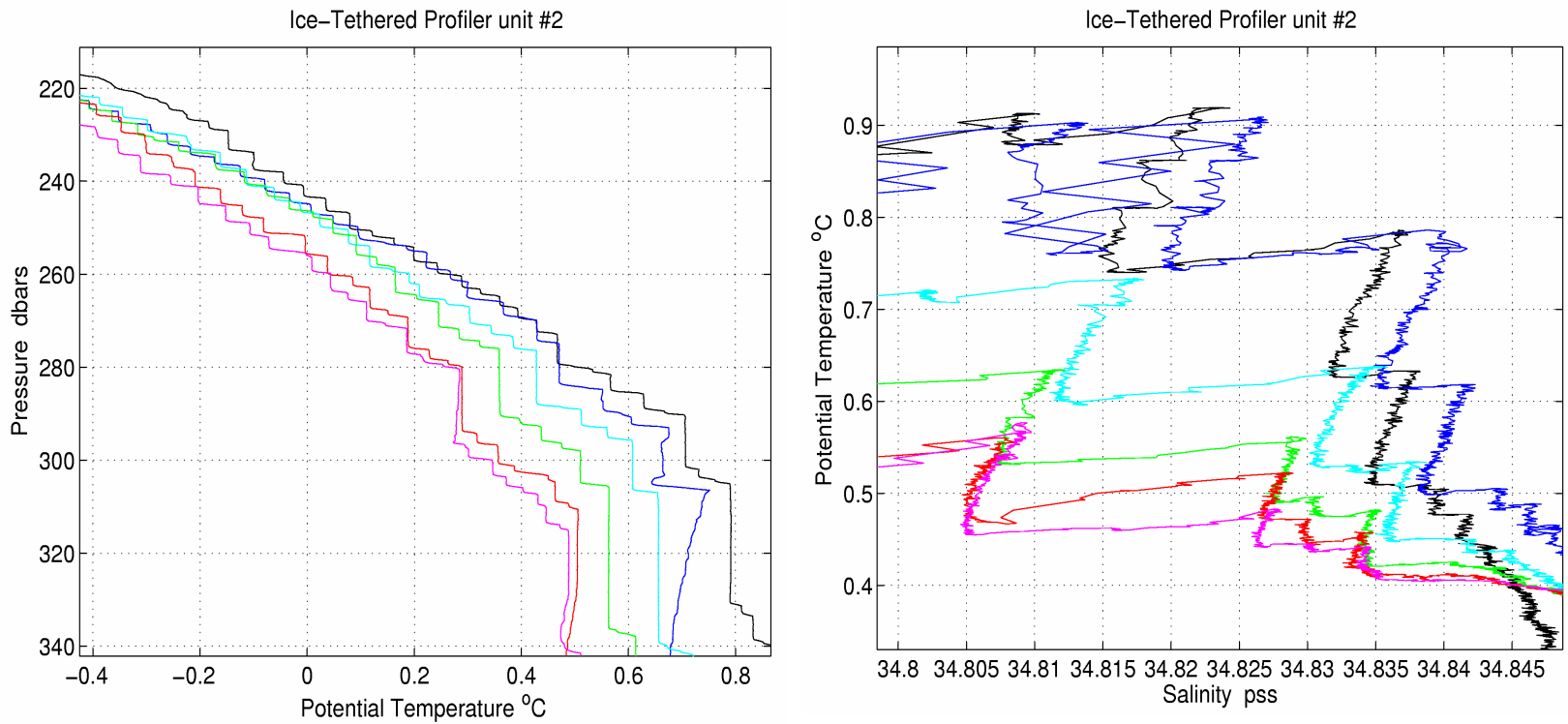

Figure 16: Expanded views of the potential temperature profiles and potential temperature - salinity diagrams. Above the Atlantic Layer, temperature and salinity both decrease with height: a stratification subject to the diffusive layering form of double diffusion that is manifested by the staircase profile resolved by the ITP (a). Overplotted potential temperature-salinity curves at the level of the Atlantic Layer (b) clearly document intrusions that erode this warm salty water mass. 
The CTD profile data so far obtained document interesting spatial variations in the major water masses of the Beaufort Gyre including the low-salinity surface mixed layer, the complex forming the Pacific Halocline Waters characterized by multiple temperature extrema between 40 and $180 \mathrm{~m}$ depth indicative of the Alaska Coastal Water, the Summer and Winter Bering Strait Waters and winter shelf waters emanating from Barrow and possibly Herald Canyons, and the temperature maximum around $350 \mathrm{~m}$ depth characterizing the Atlantic Water (Figure 15).

Additionally, the recovered $1 \mathrm{~Hz}$ CTD data resolve well the thermohaline staircase stratification above the Atlantic Layer thought to be caused by double diffusion and the "nested" intrusive structures that incise the Atlantic Water (Figure 16). CTD data from the staircase stratification has additionally proven valuable for quantifying sensor response mismatches in the SBE CTD. Due to the placement of the CTD at the top of the Profiler, these small-scale structures are poorly resolved on down profiles when the CTD inlet lies within the wake of the Profiler body. Ideas for alternative CTD ducting are under discussion.

\section{FUTURE PLANS}

The practical, costeffective and proven ITP design enables a sustained Arctic observation program in the spirit of the Argo program that is now operating in the temperate oceans (http://www.argo.ucsd.edu/). We envision a loose array of approximately 20 of these ITPs being maintained throughout the ice-covered Arctic Ocean to observe the annual and interannual variations of the upper ocean (Figure 17). Being lowcost, systems should be able to be deployed and maintained in sufficient numbers to map the upper Arctic Ocean thermohaline

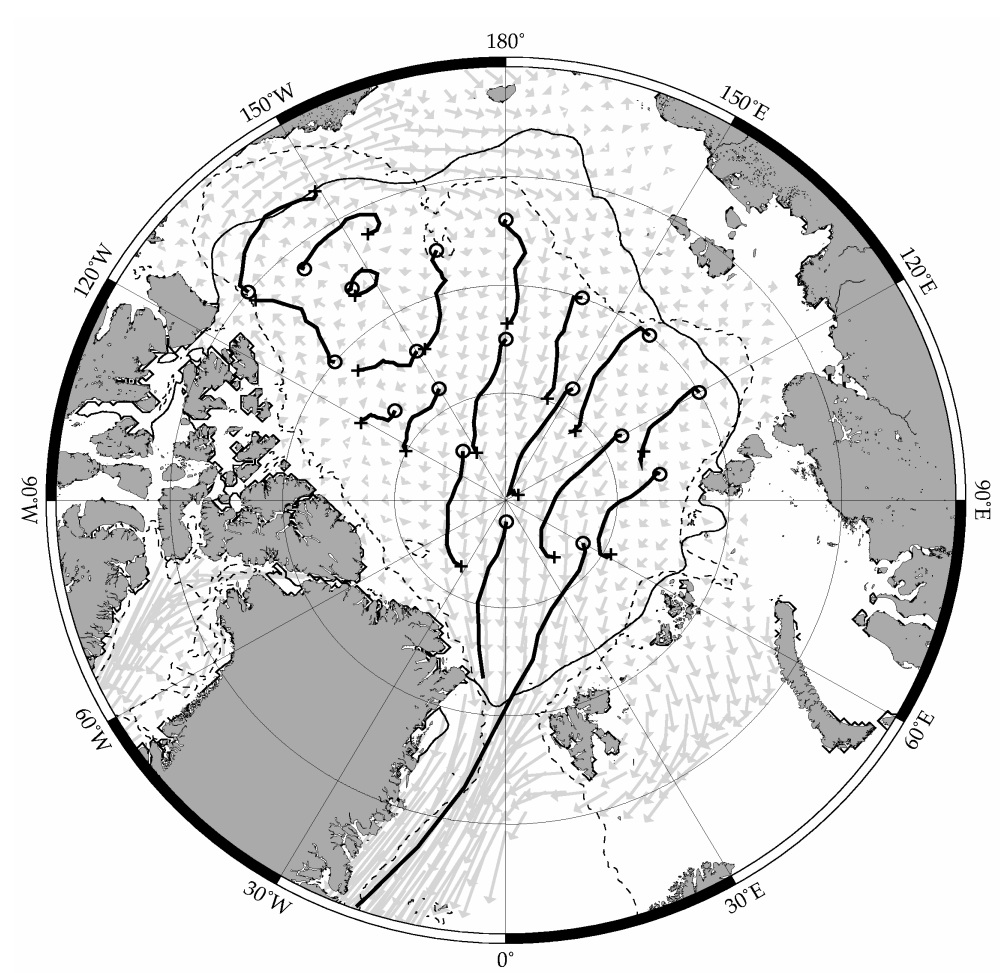

Figure 17: Simulated drift tracks for one year from 20 ITPs deployed in the Arctic at the circles and forced with climatological monthly ice drift vectors from the IABP. 
properties at daily to weekly time scale. High vertical resolution data will also be valuable for documenting and assessing double-diffusive layering, thermohaline intrusions and mesoscale eddies, all possibly important to the evolution of the Arctic ice-ocean system.

In collaboration with the polar research community, we have begun initializing an array of IBOs by deploying ITPs in conjunction with CRREL IMB buoys and Naval Postgraduate School heat flux buoys. With support from the NSF Arctic Sciences Section, 4 new ITP systems are presently being prepared for deployment on the JWACS cruise in 2006 and on the NPEO expedition in 2007. Plans to augment the array for multiple years have been accepted by the ICSU/WMO Joint Committee for the International Polar Year 2007-2008 (Toole, "An Array of Arctic ITPs"; EoI \#905), and submitted to NSF Office of Polar Programs, and to the European DAMOCLES community. Data from the array will provide the basis for operational activities as well as process studies and model validation/assimilation research, work that will ultimately lead to better understanding of the Arctic Ocean's response and role in global climate change.

One additional ITP being constructed in 2006 using internal WHOI funding will include a dissolved oxygen sensor on the profiler. Addition of other sensors (such as a current meter, PAR, fluorometer, and turbidity sensor) and additional fixed-depth modules such as surface meteorological sensors and subsurface acoustic systems are also being considered. In the future we will also exploit the two-way capability of the transmitter to allow users to modify the sampling schedule of the Profiler or other instrumentation that may be interfaced to the ITP over the RS-422/RS-485 network. The combination of subsurface acoustics with the Iridium telemetry could constitute a telecommunications link through the surface ice pack and serve as a future backbone for two-way transmissions to buoys, AUVs, and subsurface moorings in the Arctic Ocean. 
Acknowledgments. Many WHOI machinists, technicians and engineers provided expertise to this project including: Neil McPhee for building the prototype ITP electronics, Kris Newhall who participated in the deployment cruises, Jim Ryder who built the reinforced tethers, Steve Lerner who provided the logger computer software, and Chris Linder who provided the photos of the deployment operations and maintains the web page. The adaptation of the MMP engine to the ITP profiler was performed by Mike Mathewson, Gwenyth Packard, Tim Shanahan, and Mark Belcher at McLane Research Laboratories. We also acknowledge the technical assistance provided by Sea-Bird Electronics, and Webb Research Corporation during the development effort. We are grateful to our colleagues from Institute of Ocean Sciences (Eddy Carmack, Fiona McLaughlin, Sarah Zimmermann, and Doug Sieberg) and from Japan Agency for Marine-Earth Science and Technology (Koji Shimada and Motoyo Ito) for collaborating on the JWACS field programs, and the Canadian Coast Guard and the Captains, officers, and crews of the CCGS Louis S. St. Laurent for icebreaker and helicopter support during the deployments of the prototype ITPs. Initial development of the ITP concept was supported by the Cecil H. and Ida M. Green Technology Innovation Program. Funding for construction and deployment of the prototype ITPs was provided by the National Science Foundation Oceanographic Technology and Interdisciplinary Coordination (OTIC) Program and Office of Polar Programs (OPP) under grant number OCE-0324233. Continued support has been provided by the OPP Arctic Sciences Section under award number ARC-0519899 and internal WHOI funding. Any opinions, findings, and conclusions or recommendations expressed in this publication are those of the authors and not necessarily reflect the views of the National Science Foundation. 


\section{References:}

Dickson, B., The integrated Arctic Ocean Observing System (iAOOS): an AOSB-Clic Observing Plan for the International Polar Year, Oceanologia, 48 (1), 5-21, 2006.

Doherty, K.W., D.E. Frye, S.P. Liberatore, and J.M. Toole, A Moored Profiling Instrument, J. of Atmos. and Oceanic Tech., 16, 1816-1829, 1999.

Gobat, J.I. and M.A. Grosenbaugh, WHOI Cable v2.0: time domain simulation of moored and towed oceanographic systems, Woods Hole Oceanographic Institution Technical Report, WHOI-2000-08, 89 pp., 2000.

Kikuchi, T., K. Hatakeyama, K. Shimada, T. Takizawa, and J. Morison, Oceanographic observation under the multi-year ice of the Arctic Ocean using J-CAD (JAMSTEC Compact Arctic Drifter), Proceedings of Mombetsu -02 Symposium, Mombetsu, Hokkaido, Japan, 2002.

Honjo, S., T. Takizawa, R. Krishfield, J. Kemp, and K. Hatakeyama, "Drifting Buoys Make Discoveries About Interactive Processes in the Arctic Ocean", EOS Trans. AGU, 76, pp. 209, 215, 219, 1995.

Morison, J.H., S.P. Burke, H. Steltner and R. Andersen, SALARGOS temperature-conductivity buoys, Proceedings of Oceans '82, IEEE, 1255-1260, 1982.

Morison, J. H., K. Aagaard, M. Steele, Recent Environmental Changes in the Arctic: A Review, Arctic, 53, 4, 2000.

Morrison, A.T., III, J.D. Billings, and K.W. Doherty, "The McLane Moored Profiler: An Autonomous Platform for Oceanographic Measurements," Proceedings of OCEANS 2000, MTS/IEE/OES, Vol. I, 353-358, 2000.

Proshutinsky, A., A. Plueddemann, J. Toole, R. Krishfield, eds., Ice-Based Observatories: A strategy for improved understanding of the Arctic atmosphere-ice-ocean environment within the context of an Integrated Arctic Observing System, Woods Hole Oceanographic Institution, Woods Hole, Massachusetts, 65 pp., 2004.

SEARCH SSC, SEARCH: Study of Environmental Arctic Change, Science Plan, Polar Science Center, Applied Physics Laboratory, University of Washington, Seattle, 89 pp., 2001. 


\section{Appendix A: SURFCON Wiring Diagrams}

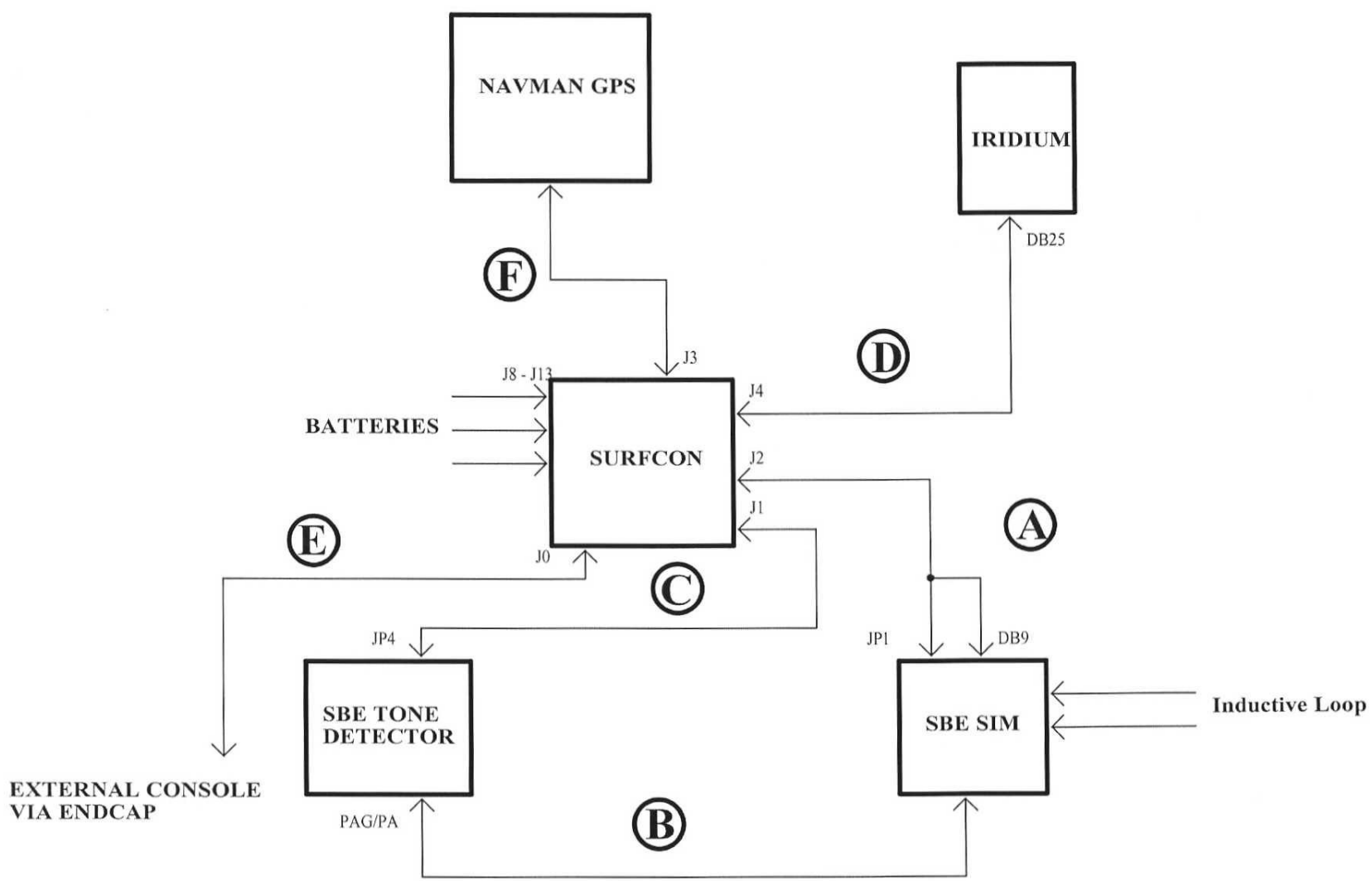

Note: Do not use pin number markings on AmpModu

Ice Tethered Profiler housings as a reference!

Surface Controller

Block Diagram

Jumpers must be properly set on SURFCON and SIM boards.

Surface mount R13 should be removed from SBE TD board.

Phone card with dial up data modem capability must be installed in Iridium ISU. 

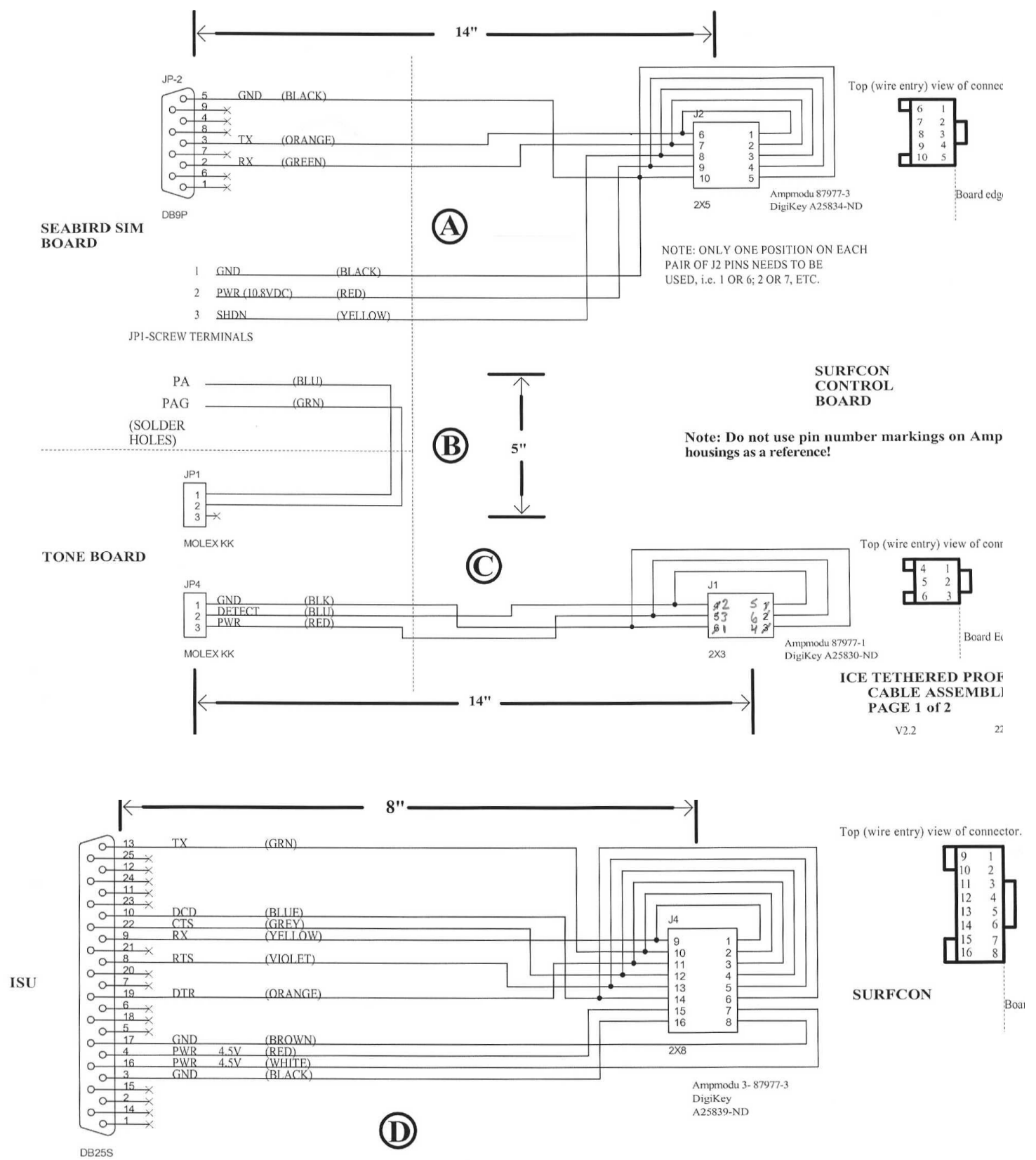

DB25S

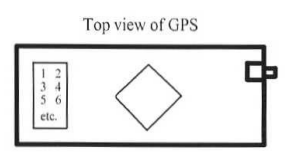

Note: Do not use pin number markings on AmpModu housings as a reference!
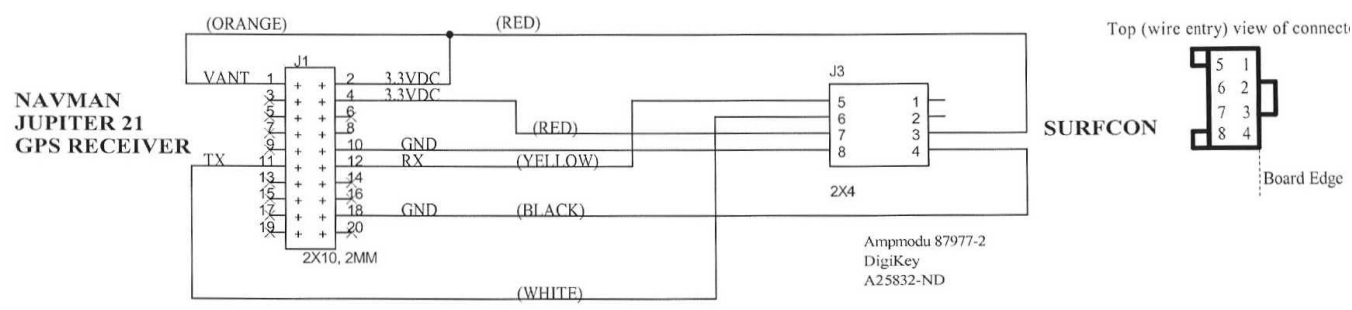

()

ICE TETHERED PROF CABLE ASSEMBLIE: PAGE 2 of 2 


\section{Appendix B: ITP Profiler wiring connections}

\begin{tabular}{lll} 
MMP/Host & \multicolumn{3}{l}{ Communications Port1: } \\
COM1 & J3-2 & RSR1 (MMP RxD) \\
& J3-3 & RST1 (MMP TxD) \\
& J3-4 & DGND
\end{tabular}

MMP/Sea-Bird CTD/Pump Power \& Communications (CMOS Voltage Levels):

FREQ

$\begin{array}{ll}\text { J7-1 } & \text { DGND } \\ \text { J7-2 } & \text { FREQ1 (MMP TxD) } \\ \text { J7-3 } & \text { FREQ2 (MMP RxD) } \\ \text { J7-6 } & \text { VSW4 (CTD/Pump POWER) }\end{array}$

MMP/Sea-Bird UIM Power:

\begin{tabular}{|c|c|c|}
\hline ANALOG & $\begin{array}{l}\text { J8-1 } \\
\text { J8-9 }\end{array}$ & $\begin{array}{l}\text { DGND } \\
\text { VSW3 (UIM POV }\end{array}$ \\
\hline MMP/Sea & JIM Cc & Imunications Port \\
\hline FREQ & J7-4 & RSR2 (MMP RxD \\
\hline & $J 7-5$ & RST2 (MMP TxD \\
\hline MMP/Sea-B & JIM To & Detect Discrete: \\
\hline XPONDER & J6-1 & DGND \\
\hline & $\mathrm{J} 6-2$ & TP8 \\
\hline MMP/Moto & & \\
\hline MOTOR & $\mathrm{J} 2-1$ & $(+)$ \\
\hline & $\mathrm{J} 2-3$ & $(-)$ \\
\hline MMP/Powe & & \\
\hline BATTERY & J1-1 & NEG (DGND) \\
\hline & $\mathrm{J} 1-2$ & POS1 (10.8Vdc) \\
\hline & $\mathrm{J} 1-3$ & POS2 (10.8Vdc) \\
\hline & J1-4 & POS3 (10.8Vdc) \\
\hline & $\mathrm{J} 1-5$ & POS4 (10.8Vdc) \\
\hline & $\mathrm{J} 1-6$ & POS5 (10.8Vdc) \\
\hline & $\mathrm{J} 1-7$ & POS6 (10.8Vdc) \\
\hline & $\mathrm{J} 1-8$ & POS7 (10.8Vdc) \\
\hline & J1-9 & POS8 (10.8Vdc) \\
\hline & $\mathrm{J} 1-10$ & NEG (DGND) \\
\hline
\end{tabular}




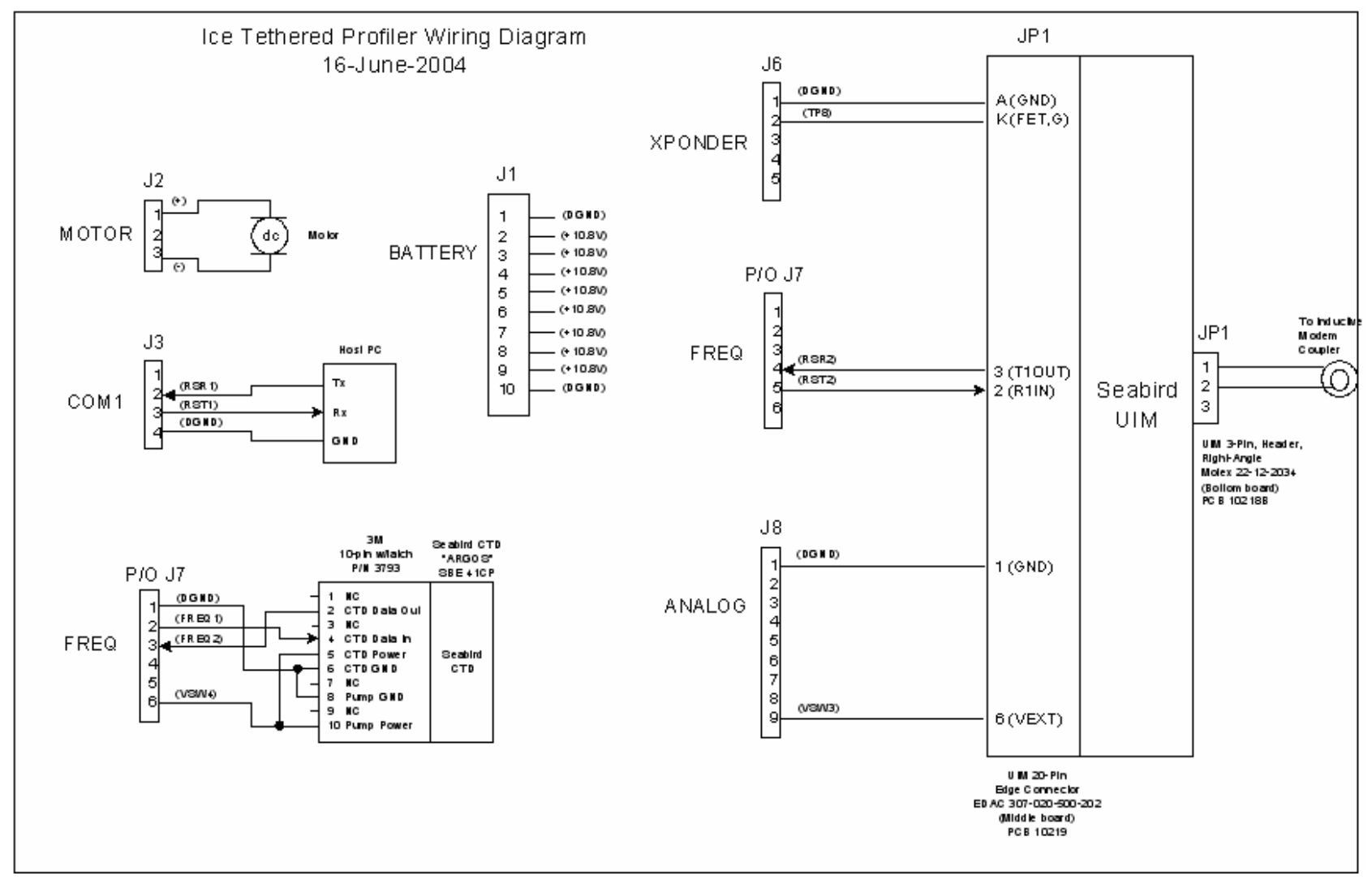




\section{Appendix C: Operating the ITP surface package}

\section{Console connection}

Do not connect a standard DB9P cable to the SURFCON console port if more than TX, RX and GND are wired as the connection is designed to accept a reset connection as well as communications. Either use the special console cable with the pushbutton reset switch on the DB9S end or an adapter to make sure that pins other than 2, 3 and 5 are not connected. SURFCON is DCE so it connects directly, without null modem, to a DTE computer serial port. A longer console cable for use in the field connects to the 5 pin water tolerant connector emerging from the top of the ITP package, which should permit communications with the system from a helicopter seat in the field while checking operation just after deployment. Fifty feet is a practical limit for standard RS232 comms over such a cable. There is a manual RESET switch on the SURFCON board which does the same thing as the pushbutton at the end of the console cables. This is more than just a reset directly connected to the Persistor as it must be able to awaken the system from sleep mode when the Persistor is unpowered. The effect of the button is to awaken SURFCON as would the WAKE from the TONE DETECTOR, which causes SURFCON to be powered up, then after a 1.6s delay, hits it with a hard reset resulting in a command line prompt at the console rather than continuing where the program code left off...if a program had been running when it entered sleep mode. Depending on the state of the SURFCON processor, a RESET may be indicated on the console as "TD" wakeup.

\section{Configuration}

There are 2 programs at this time. XBT is a test program and SURFCON is the acquisition program. Configuration information for the SURFCON operating parameters are stored in an ASCII text file "ITPSYS" on the Persistor flash card. An AUTOEXEC.BAT file in the root directory containing the line "surfcon" causes the program to run on powerup. There is a watchdog facility that will cause a reboot should nothing happen for about 20 minutes. Prior to deployment, there are a few software items that should be configured, as follows:

- The root directory file "ITPSYS" should be edited (captured or pasted) to reflect the desired parameters shown below. It is important that they are in this order on separate CRLF terminated lines and the parameters themselves must be immediately preceded by a colon ' $:$ '.

"Itpsys number" specifies the serial number of the ITP system; 
"Status epochs per day" specifies the number of equally spaced times per day that the ITP will assemble status information including a GPS fix to add to the daily status file;

"Console active time" specifies the time in seconds that SURFCON state information will be written to the console. After this time has elapsed, very little information will be sent to eliminate the power used by the RS232 drivers since for most of its life, nobody will be watching;

"Max daily ISU time" specifies the daily limit in seconds that ISU operations are permitted to reduce the risk that excessive ISU power will be expended. The times must be set to comfortably allow transmissions of the expected amount of data at approximately $250 \mathrm{Byte} / \mathrm{sec}$ plus about 2 minutes per expected connection. In addition the time should be sufficient to allow sending a few days worth of data in case the iridium link cannot be established possibly due to temperature below -30 C.

"Initial ISU transmit delay" typically 0, so that ISU transmission can be forced if desired at start up.

"Max Daily IM Wakeups per day" specifies a limit on the occurrences of TE wakeup due to UIM activity, to reduce power consumption should spurious UIM wakeups occur repeatedly. SURFCON is still awakened but immediately returns to the suspended state rather than power up the SIM and wait for DATA while the timeout elapses.

"ISU transmission epochs" specifies that the number of occasions equally spaced in time that SURFCON will attempt to establish an Iridium connection if there are files to send. This is another measure to limit energy use

A sample ITPSYS file:

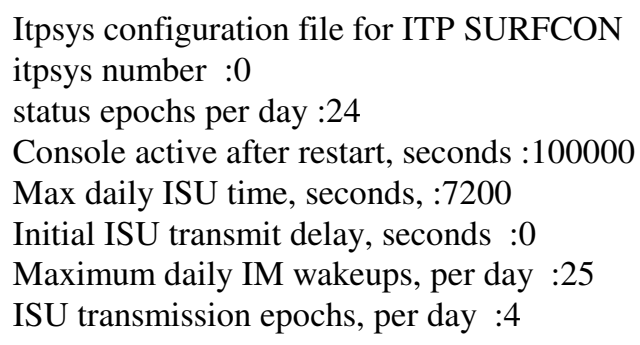

- Two system environment variables must be set. Typing "SET" by itself will return the settings, which should be:

○ SYS.BAUD=19200,N 


\section{$\circ \quad$ SYS.QPBCS=3}

If these variables are set to any other values, the program will not function. Changing the values of these variables is done by typing, "SET SYS.BAUD=19200, N" and "SET SYS.QPBCS=3" at the command line. All environment variables can be cleared by typing "SET $=<\mathrm{CRLF}>$ ".

- The root directory file "RESTARTS" should be deleted, which will force the start of a new one. The sole purpose of this file is as a place to keep count of the number of times that the controller reboots, which typically in the field would be caused by a WatchDog Reset. Its value is reported in the daily STATUS file.

- $\quad$ Make sure an AUTOEXEC.BAT file exists in the root directory with the sole line "SURFCON" followed by a return.

- $\quad$ Delete garbage and test files from the root, $\mathrm{LOG}, \mathrm{ICMD}$ and $\mathrm{DATA}$ directories.

\section{Board Test}

XBT is an executable program to exercise functions of SURFCON with a serial console connected, for diagnosing faults in the hardware, and for testing exchanges with PROCON.

Usage: xbt -options

ITP Surface Controller board tests. Select \& hit return

f....Continuously open and write small files in default dir

a....Run 8 channel ADC and report converted values

p....Turn on selected device and run serial port thru console

s....Suspend SURFCON and wait for /TD_WAKE or /EXT_RESET

m....Place ISU call \& send test message

g....Output GPS RMC string

t....Read GPS date/time and set RTC

u....Power up SIM \& UIM, read status from UIM

c.....Monitor ISU signal strength

1....Dial up and login to logger \& immediately bailout

q....Quit

Options list:

$-\mathrm{H}$

printf this menu

-cont interval Applies to 's' suspend, 'g ' GPS, and 'm' message menu options

If 's' ops, suspend, recover, give testor a chance to exit, repeat

If ' $\mathrm{g}$ ' ops, powerup ISU, read RMC lines until fix, ISU off, repeat

If ' $t$ ' ops, same as ' $\mathrm{g}$ ' except RTC is set w/ GPS time

If ' $m$ ' ops, ISU on, send test message, repeat

The intention is to test reliability of ISU turn-on which

has been problematic as temperature decreases.

The interval is in seconds and needs to be $>=300$ to get to $-20 \mathrm{C}$

-susp

If doing continuous ops, suspend during wait period

Works for ' $m$ ' and ' $\mathrm{g}$ ' tests 


$\begin{array}{ll}\text {-gto timeout } & \begin{array}{l}\text { Set the wait time for a valid GPS fix. This has been used } \\ \text { primarily to speed up the ISU turnon check using the 'g' menu test } \\ \text { Just set gpstimeout to something small like 2, default is 120s which } \\ \text { should usually be more than enough time to get a valid fix }\end{array} \\ \text { Do not get GPS info for test file, menu option } \\ \text {-nogps } \\ \text {-crc initval } & \text { Compute 16 bit CCITT CRC for testfile using spec'd init value } \\ \text {-creq } & \text { After a test file transmission 'm', send CREQ to get MESG pkt from logger } \\ \text {-dial 10digit\# } & \text { Specify 10 digit US phone number, no intervening spaces } \\ \text {-reqdir } & \text { Specify reqdir command for after suspend ops } \\ \text {-reqfil fname } & \text { Specify reqfil command for after suspend ops } \\ \text {-reqnew } & \text { Specify reqnew command for after suspend ops } \\ \text {-scto \# } & \text { Specify SIM intercharacter timeout in ms <2000> } \\ \text {-user username } & \text { Specify username to which Linux logger will respond <itpsys1> }\end{array}$

To deactivate ISU PIN, first enable using current pin (default=1111), at+cpin="1111"

Then issue: at+clck="sc",0,"1111" where 1111=old pswd

To power and give direct commands to the Iridium transceiver command $\mathrm{P}$ is used:

Selection....p

Select port, I=ISU, S=SIM, L=logic, 2=spare RS232, 4=RS422, ^ X to QUIT....

Select baudrate, 0 for default --> ISU:19200, else $9600 \ldots 19200$

ISU port Rx chan is TPU4, Tx chan is TPU3

$\#$ bits $=8$

parity $=\mathrm{N}$

baudrate $=19200$

Receive channel TPUPriority $=-1$

Transmit channel TPUPriority $=-1$

Receive queue size $=1024$

Transmit queue size $=1024$

Transmit channel printf buffer size $=512$

Selected port should be open@19200 baud

*** Break from PORT test with ${ }^{\wedge} \mathrm{X}$

at

OK

at+creg?

+CREG:000,004

$\mathrm{OK}$

at+creg?

+CREG:000,001

at+cpin?

+ CPIN:SIM PIN2

$\mathrm{OK}$

at+csq?

$+\mathrm{CSQ}: 4$

at+cgmi

Motorola

at+cgmm

9522 Satellite Series

at+cgmr

Call Processor Version: SAC0309

DSP Version: SAC0108

NVM Version: SAC0200

at+cgmi

Motorola 
at+cgsn

To obtain a GPS location, enter command G:
Selection....g
Select baudrate, 0 for default of 4800 , else as specified....4800
Using logic level port for GPS, baudrate $=4800$
\$GPRMC,184357,V,0000.0000,N,00000.0000,E,0.000,0.0,130406,0.0,E*6D
\$GPRMC,184358,V,0000.0000,N,00000.0000,E,0.000,0.0,130406,0.0,E*62
\$GPRMC,184359,V,5047.2079,N,07445.4188,W,0.000,0.0,130406,0.0,E*7
\$GPRMC,184400,V,5047.3811,N,07445.1414,W,0.000,0.0,130406,0.0,E*75
\$GPRMC,184401,A,4132.0785,N,07038.9015,W,0.000,0.0,130406,15.3,W*46
\$GPRMC,184402,A,4132.0658,N,07038.8932,W,0.000,0.0,130406,15.3,W*49
\$GPRMC,184403,A,4132.0675,N,07038.8881,W,0.177,148.2,130406,15.3,W*40
\$GPRMC,184404,A,4132.0656,N,07038.8895,W,0.159,150.7,130406,15.3,W*43

To set the RTC time to the GPS time, enter command T:

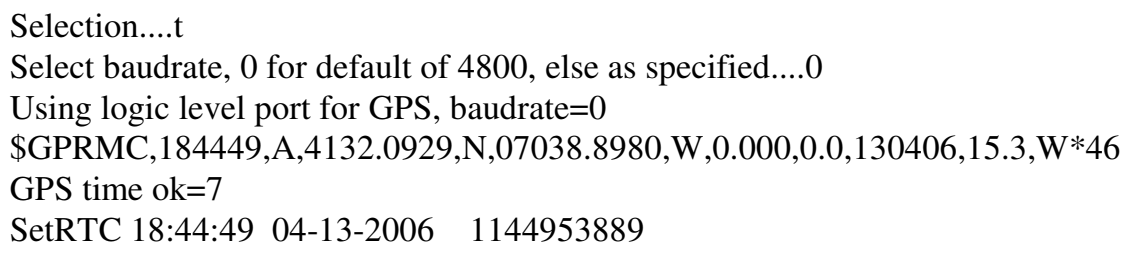

To automatically dial up and hang up with the Iridium transceiver, enter command L:

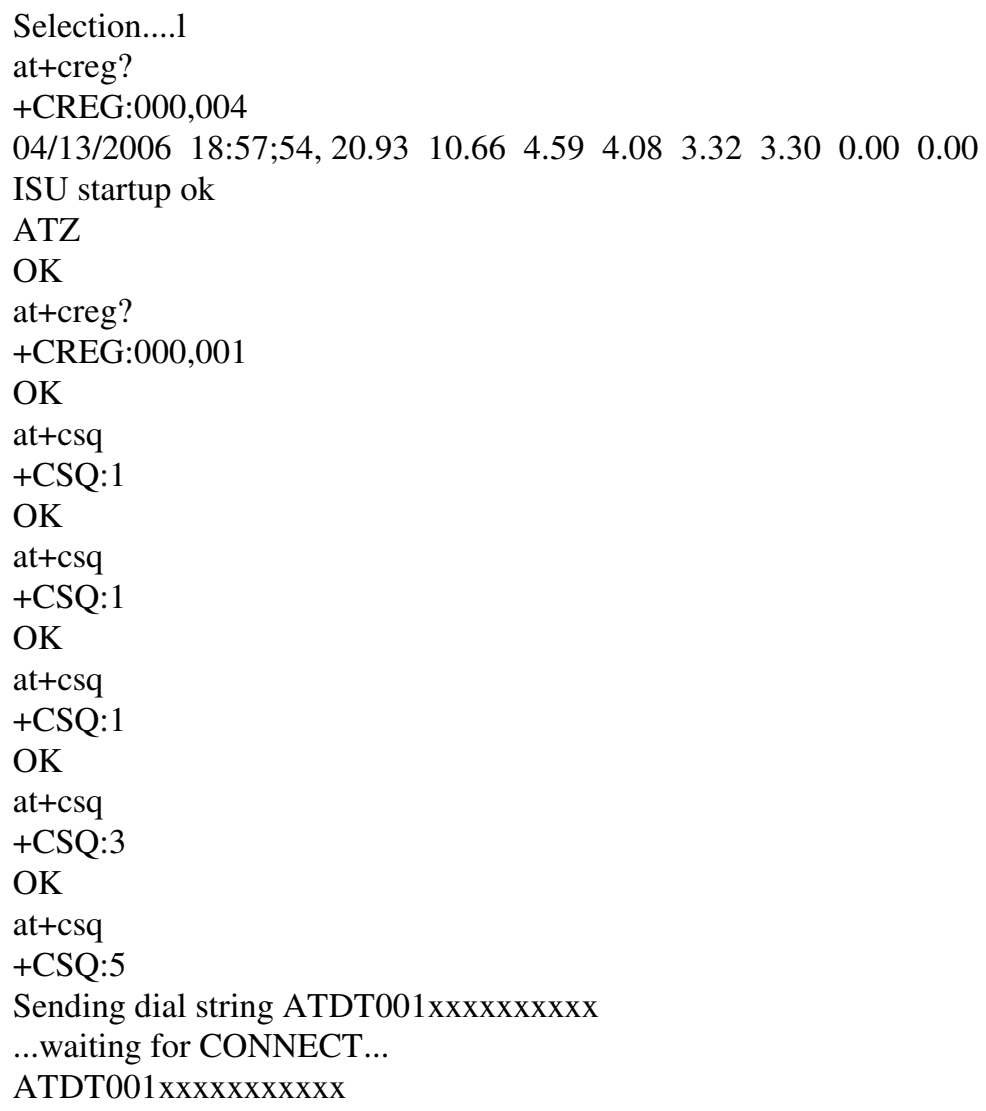


CONNECT

04/13/2006 18:58;45, Received CONNECT in 14 seconds, attempt 1

$\operatorname{login}$

04/13/2006 18:59;07, Received LOGIN in 22 seconds, sigstren=5 attempt 1

\section{Inductive Modem Testing}

In order for the SIM and UIM to communicate properly, the SIM should initially be off, the UIM should turn on (nominally after obtaining a profile) and waken the SIM with a $4800 \mathrm{~Hz}$ tone. If both the SIM and UIM are listening on the inductive loop at the same time, both units will report garbage characters.

When an ITP is deployed, XBT command U can be used (after breaking out of the SURFCON program) to verify the inductive modem communications with the deployed profiler. The profiler UIM remains on until the first profile (down profile 0), so will respond to DS commands:

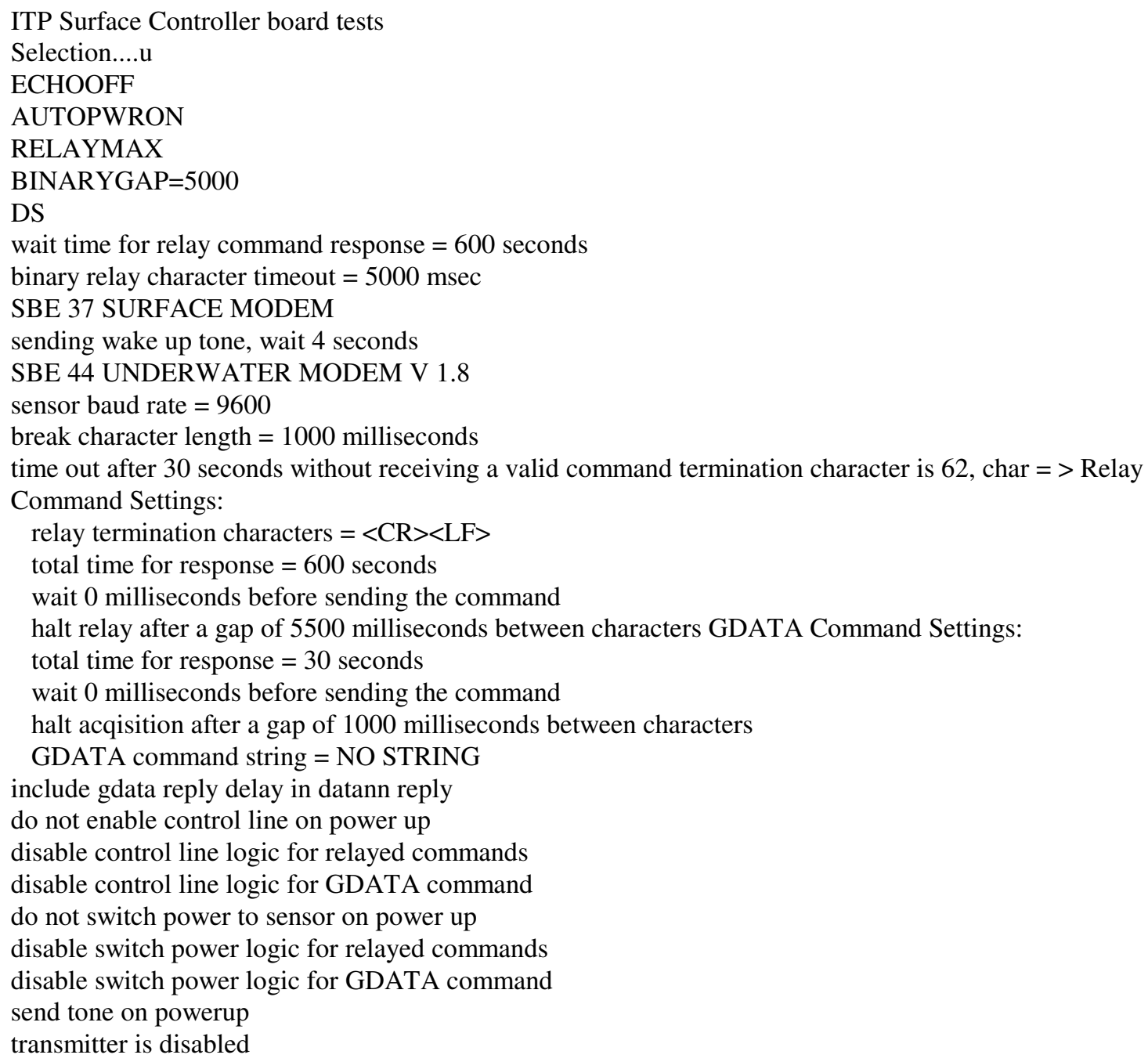




\section{Operating program}

Surface controller data acquisition program (SURFCON) is normally called automatically upon power up or reset of electronics within an AUTOEXEC.BAT command script, and with all default settings by invoking no options.

\begin{tabular}{|c|c|c|}
\hline \multicolumn{3}{|l|}{ Options list: } \\
\hline -contime & \# & Time console output will be active after startup $<14400$ s $>$ \\
\hline -simto & \# & Specify SIM TIMEOUT in sec $<3600>$, then quit \\
\hline -stepoch & \# & Specify number of status data collections per day $<4>$ \\
\hline -nogps & & Do not operate the GPS \\
\hline -gto & \# & Specify time to get GPS time/fix each occasion $<300>$, then quit \\
\hline -DIAL1 & \# & Specify primary number for ISU dial, 10 digit \\
\hline -DIAL2 & \# & Specify secondary number for ISU dial, 10 digit \\
\hline -TXDLY & \# & $\begin{array}{l}\text { Specify delay of forced initial ISU transmission attempt, sec }<10>\text {; } \\
\text { Note, if no file in FILELIST, there will be no transmission }\end{array}$ \\
\hline -MAXDIM & \# & Specify max number of accepted IM Tone detect wakeups per day $<10>$ \\
\hline -ISUTXEP & \# & Specify the number of times per day the ISU will attempt transmissions $<4>$ \\
\hline
\end{tabular}

SURFCON awakens from SUSPEND mode either as a result of TD or internal RTC alarm at the GPS (status epoch) or telemetry (transmission epoch) intervals. SURFCON initiates status transmissions to home daily regardless of the operation of the profiler. Data that arrives from PROCON is transmitted the number of times per day specified by the transmission epoch.

TD wakeup will under some conditions, have priority over other operations that might be in progress. If the PROCON attempts to awaken the SURFCON when the latter is assembling a status entry, SURFCON will complete the status entry as it will often finish before the PROCON times out in its attempt to contact SURFCON. However if an ISU transmission is in progress, SURFCON will check for PROCON contact and break out if a TD is detected. The rationale is that we don't want to miss a status check and the time to do so is usually less than $45 \mathrm{~s}$ whereas an ISU transaction can be much longer and can easily be resumed where it was disconnected..

Fundamentally, SURFCON initiates all ISU transactions at least once daily according to the parameter ISU_transmission_epochs which specifies the number of times per day ISU ops can be attempted should there be files in the queue. The actual transmission times are offset in time as a function of the ITPSYS\# to minimize dialup conflicts as all ITPs currently call one of 2 phones. There should always be at least 1 status file per day. Files are assumed to be binary and are sent as packets that include a short header followed by a payload of 4096 bytes. The header consists of the ASCII characters "DATA", and 3 ASCII numbers specifying the packet sequence number, the payload length and a 4 hex digit algebraic checksum. A file transmission is announced by sending a FILESPEC packet which consists of the keyword "FILE", a space, the filename in 8.3 format, a space and the file length in decimal ASCII characters. The logger 
running on the receiving server responds with an "RSTR" packet that indicates whether part of the file has already been received during a previous session and which packet should be received next or if it thinks this is a new file in which case the current transmission will begin with the first packet.

Each packet is acknowledged by the server and the SURFCON waits for an acknowledge packet when 2 data packets remain unacknowledged. The operation is subject to timeout and retry limits on each occasion as well as a limit on total ISU time per day. Typical net data thruput is about 300 bytes per second once the overhead of dial-in is achieved which can take a minute or more

The daily SURFCON status file are always sent home at the first transmission time after $0000 Z$ on the next day which includes ITPSYS number, date, time, GPS fixes at the preset interval, SURFCON power voltages, internal temp, SURFCON date/time of last communication from PROCON, and total time of ISU usage for the day. The state of the TD wakeup is monitored during status transmission operations to preserve priority for response to the PROCON.

Upon awakening by PROCON, SURFCON requests file; if timeout, SURFCON suspends; else PROCON sends packet; if timeout for packet tx, SURFCON sends NAK; else if packet error, SURFCON sends NAK; else if ok, SURFCON sends ACK; if on $2^{\text {nd }}$ transmission attempt of a given packet, packet error, then SURFCON sends NAK; if no response to ACK or NAK, repeat; if no response to $2^{\text {nd }} A C K$ or NAK, SURFCON suspends. SURFCON always waits for at least $1500 \mathrm{msec}$ beyond last char received from PROCON to make sure UIM has switch to binary receive mode.

When a command file from the logger is received, an "ACK" file is generated by the SURFCON as acknowledgement of command receipt and sent immediately back to the logger with filename of the form mmddhhnn.ACK.

SDIR, SFIL and SCMD commands are executed immediately upon receipt and if a file results, it is sent in the current session with the logger.

PDIR and PFIL commands are accepted and held for the next session with the Profiler Controller, hence any results will at the earliest, be returned to the logger at the end of the next Profiler session. 
The directory structure that is created by SURFCON is:

\section{C:Icmd All “.ACK”, ".PDR” and ".SDR” files}

\section{C:Idat All PROCON “.DAT” files and SURFCON ".STA” files}

\section{C: $\log \quad$ All “.LOG” files of software operations}

\section{A sample ISU transaction excerpted from screen log:}

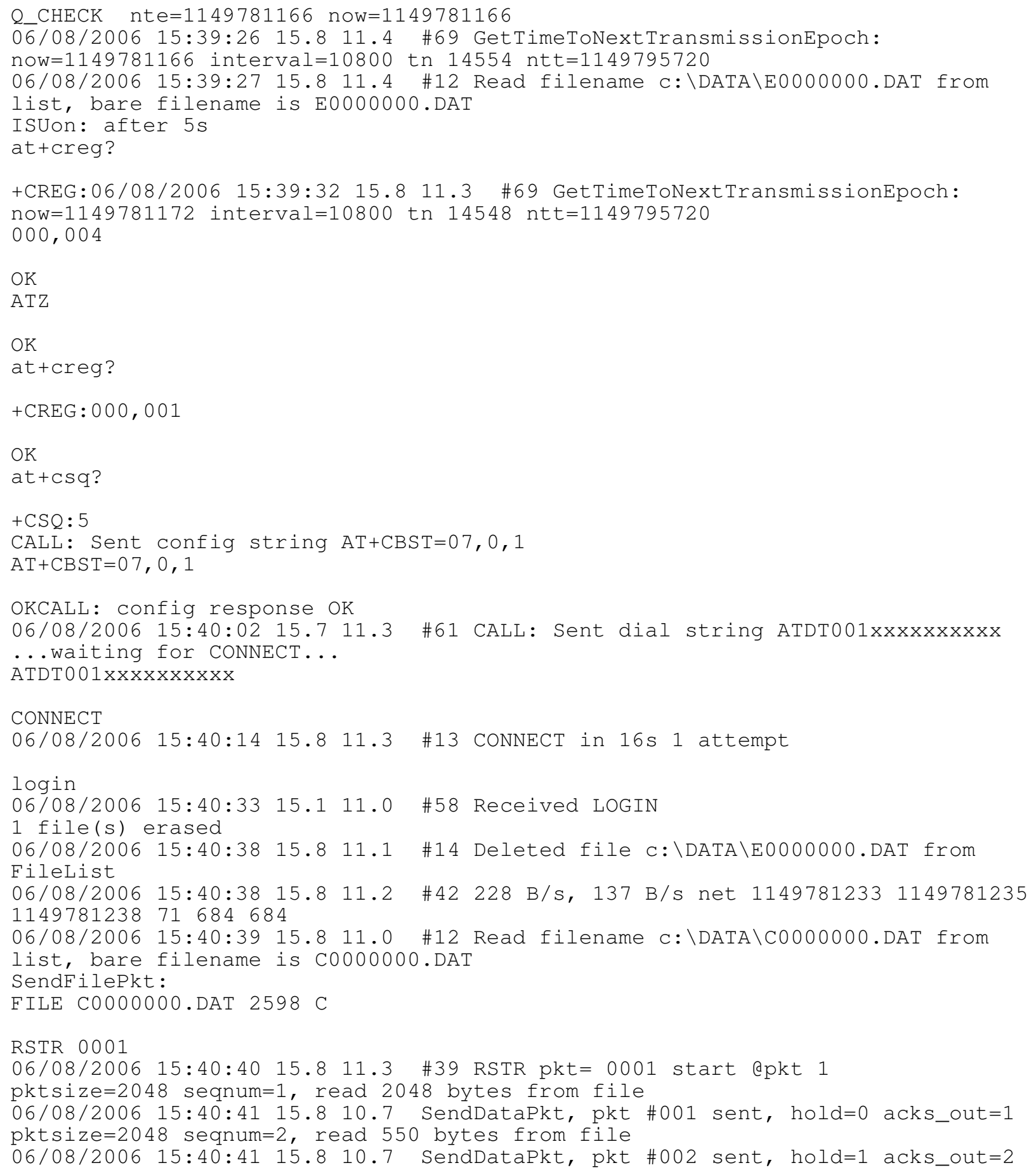


1 file(s) erased

06/08/2006 15:40:49 15.8 11.3 \#14 Deleted file c:\DATA \C0000000.DAT from

FileList

06/08/2006 15:40:49 15.8 11.3\#42 $289 \mathrm{~B} / \mathrm{s}, 205 \mathrm{~B} / \mathrm{s}$ net 11497812331149781240

$1149781249 \quad 82 \quad 3282 \quad 2598$

1 file(s) erased

looking for MESG pkt

MESG CREQ: Got MESG, looking for $\backslash n$

$0088171 \mathrm{C}$

CREQ: rec'd 11 chars, got $\backslash \mathrm{n}$

06/08/2006 15:40:50 15.9 11.2 \#43 CREQ: MESG payload len=88

SDIR C: \

found dir $c: \backslash$

06/08/2006 15:40:50 15.9 11.3 \#45 Loaded file \CMD\06081540.SDR with SC

directory info

06/08/2006 15:40:50 15.8 10.8 \#10 Added file \CMD 106081540. SDR to FileList

06/08/2006 15:40:51 15.8 11.3 \#46 CREQ: Adding SDIR

filespec $=\backslash C M D \backslash 06081540 . S D R$ to Inbound list

06/08/2006 15:40:51 15.8 11.3 \#52 CREQ: writing cmd acknowledgment file, cmd sequence SDIR c: \

received:

SFIL c: \itpsys

06/08/2006 15:40:51 15.8 11.2 \#10 Added file c: \itpsys to FileList

06/08/2006 15:40:51 15.9 11.2 \#44 CREQ: Adding SFIL filespec=c: \itpsys to

Inbound list

06/08/2006 15:40:51 15.1 11.1 \#52 CREQ: writing cmd acknowledgment file, cmd

sequence SFIL c: \itpsys

received:

SDIR C: \DATA

found dir $C: \backslash D A T A$

06/08/2006 15:40:52 15.8 11.0 \#45 Loaded file \CMD\06081540.SDR with SC

directory info

06/08/2006 15:40:52 15.8 11.3 \#10 Added file \CMD 106081540. SDR to FileList

06/08/2006 15:40:52 15.8 11.3 \#46 CREQ: Adding SDIR

filespec $=\backslash C M D \backslash 06081540 . S D R$ to Inbound list

06/08/2006 15:40:52 15.7 10.8 \#52 CREQ: writing cmd acknowledgment file, cmd sequence SDIR C: \DATA

received:

PFIL IRQ_XCPT.LOG

$06 / 08 / 2006$ 15:40:52 15.9 11.3 \#49 CREQ: PFIL filespec=IRQ_XCPT.LOG

reqfilnames $=$ IRQ_XCPT.LOG, len $=13$

06/08/2006 15:40:52 15.8 11.1 \#52 CREQ: writing cmd acknowledgment file, cmd sequence PFIL IRQ_XCPT.LOG

received:

PFIL LASTSENT.DAT

06/08/2006 15:40:52 15.9 11.2 \#49 CREQ: PFIL filespec=LASTSENT.DAT

reqfilnames $=$ IRQ_XCPT.LOG LASTSENT.DAT, len=26

06/08/2006 15:40:53 15.7 10.7 \#52 CREQ: writing cmd acknowledgment file, cmd sequence PFIL LASTSENT.DAT

received:

$\mathrm{PCDS}$

06/08/2006 15:40:53 15.8 11.3 \#51 CREQ: Adding PCDS request

06/08/2006 15:40:53 15.8 10.7 \#52 CREQ: writing cmd acknowledgment file, cmd sequence PCDS

received:

EOTX06/08/2006 15:41:23 15.9 11.0 \#53 Couldn't find crlf delimiter in payload section of MESG pkt

06/08/2006 15:41:23 15.9 11.3 \#10 Added file \CMD 106081540. ACK to FileList

06/08/2006 15:41:23 15.9 11.3 \#12 Read filename \CMD\06081540.SDR from list,

bare filename is $06081540 . \mathrm{SDR}$

SendFilePkt:

FILE 06081540. SDR 737 C 
RSTR 0001

06/08/2006 15:41:24 15.9 11.3 \#39 RSTR pkt=0001 start apkt 1

pktsize=2048 seqnum $=1$, read 737 bytes from file

06/08/2006 15:41:25 15.911.2 SendDataPkt, pkt \#001 sent, hold=0 acks_out=1

1 file(s) erased

06/08/2006 15:41:27 15.9 11.3 \#14 Deleted file \CMD\06081540.SDR from

FileList

06/08/2006 15:41:28 15.9 11.2 \#42 184 B/s, 73 B/s net 11497812331149781284

$1149781288121 \quad 4019737$

06/08/2006 15:41:28 15.9 11.2 \#12 Read filename c: \itpsys from list, bare

filename is itpsys

SendFilePkt:

FILE itpsys 296 C

RSTR 0001

06/08/2006 15:41:29 15.9 11.3 \#39 RSTR pkt=0001 start apkt 1

pktsize=2048 seqnum $=1$, read 296 bytes from file

06/08/2006 15:41:29 15.8 10.8 SendDataPkt, pkt \#001 sent, hold=0 acks_out=1

1 file(s) erased

06/08/2006 15:41:33 15.9 11.2 \#14 Deleted file c: \itpsys from FileList

06/08/2006 15:41:34 15.9 11.3 \#42 59 B/s, 71 B/s net 11497812331149781289

11497812941274315296

06/08/2006 15:41:34 15.9 11.2 \#12 Read filename \CMD\06081540.SDR from list,

bare filename is $06081540 . \mathrm{SDR}$

SendFilePkt:

FILE 06081540. SDR 737 C

RSTR 0001

06/08/2006 15:41:35 15.9 11.3 \#39 RSTR pkt=0001 start apkt 1

pktsize $=2048$ seqnum $=1$, read 737 bytes from file

06/08/2006 15:41:36 15.911.2 SendDataPkt, pkt \#001 sent, hold=0 acks_out=1

1 file(s) erased

06/08/2006 15:41:41 15.9 11.3 \#14 Deleted file \CMD\06081540.SDR from

FileList

06/08/2006 15:41:41 15.9 11.2 \#42 123 B/s, 74 B/s net 11497812331149781295

$1149781301 \quad 1345052737$

06/08/2006 15:41:41 15.9 11.3 \#12 Read filename \CMD\06081540.ACK from list,

bare filename is $06081540 . \mathrm{ACK}$

SendFilePkt:

FILE 06081540. ACK 210 C

RSTR 0001

06/08/2006 15:41:42 15.9 11.3 \#39 RSTR pkt=0001 start apkt 1

pktsize=2048 seqnum $=1$, read 210 bytes from file

06/08/2006 15:41:43 15.911.3 SendDataPkt, pkt \#001 sent, hold=0 acks_out=1

1 file (s) erased

06/08/2006 15:41:44 15.9 11.3 \#14 Deleted file \CMD\06081540.ACK from

FileList

06/08/2006 15:41:44 15.9 11.3 \#42 105 B/s, 74 B/s net 11497812331149781302

$11497813041375262 \quad 210$

1 file(s) erased

looking for MESG pkt

MESG CREQ: Got MESG, looking for \n

0000 FFFF

CREQ: rec'd 11 chars, got $\backslash \mathrm{n}$ $06 / 08 / 2006$ 15:41:46 15.911 .1

$06 / 08 / 2006 \quad 15: 41: 46 \quad 15.911 .3$

$06 / 08 / 2006 \quad 15: 41: 4615.911 .2$

\#43 CREQ: MESG payload len=0

\#54 Aborted CREQ Ops, MESG payload len=0

\#16 End ISU ops, daily ISU connect time=139 
06/08/2006 15:41:46 15.9 11.4 \#70 GetTimeToNextStatusEpoch: now=1149781306 interval $=3600 \quad \mathrm{tn}=1149782400 \mathrm{ntt}=0$

06/08/2006 15:41:46 15.8 11.4 \#18 suspend for 1094 s, next status 1094 s, next ISU Tx $14414 \mathrm{~s}$

To verify or access that the files on the logger from the field, it should be possible to connect a laptop to an ISU with antenna and invoke a dial-up connection as one would do with any desktop modem via a serial port. However, the Iridium link operates at more like 2400 baud and can be hesitant, but will make it possible to login to the logger computer to observe whether files are indeed being received at home. 


\section{Appendix D: Assembly of ITP Profiler}

The main housing of the ITP Profiler is an anodized aluminium cylinder 6.5" in diameter and 48 " long, with o-ring surfaces on the faces at either end, and a snap-ring groove in the bottom end. A hole is located midway between the ends, where the drive motor protrudes. The modem core is mounted on the bottom endcap of the Profiler totalling 6" in length (plus several inches of cable), while the top endcap of the instrument supports the CTD sensors and extends 13". Springed guide wheels and nylon wire guides are clamped externally near both ends of the housing, everywhere protruding from the sides of the instrument by less than 3 ".

Assembly of the Profiler begins by positioning the main housing cylinder horizontally on a surface, secured from rolling, and with both ends accessible. The nylon clamps with guide wheels should be preassembled, and then may be mounted near both ends of the cylinder. With the (snap ring grooved) bottom of the cylinder oriented south, the springs are oriented on the west and wheels on the east. Grooves and pins in the clamps align with machined hollows on the cylinder, preventing the assemblies from slipping and keeping the orientation of the guide wheels and drive motor exact.

The drive motor and roller assembly is mounted through the hole in the middle of the main housing and oriented so that the open roller face is facing east with the bottom of the tube oriented south. A sleeve is slipped into the hole, and an o-ring cleaned, greased and placed into the groove on the motor face seal, before inserting the motor into the hole in the tube. The motor is secured on the inside by a large diameter washer and plastic nut that must be tightened firmly by hand. The two wires from the motor fit into a 3-pin connector that plugs into the PROCON electronics boards. To reverse the direction of the motor, reverse polarity by switching places of the wires.

With the motor and roller assembly in position, the electronics chassis is installed next. If the instrument is being prepared for deployment, then the battery packs should be installed and the electronics powered and initialized (see Implementation text) before the chassis is inserted into the cylinder. Position the chassis so that the two long tie rods will pass on either side of the motor during insertion, and plug in the motor connection. Slide the chassis into the tube, ensuring that the CTD connector reaches to the other end, and that the 5-pin modem/console connector feeds past the bottom end plate and is accessible from the near side. Insert the chassis into the cylinder until the bottom end plate is just beyond the snap-ring groove, and insert the snap ring. Pull the bottom of the chassis until it is snug against the snap ring to facilitate attaching the bottom endcap. 
Before the bottom endcap is attached, the inductive modem coil should be mounted on the endcap. Clean and grease the face seal o-ring (\#163) and place it in the groove on the endcap. Plug in the modem/console connector and place the endcap in position, so that the modem mount lines up with clamp mount, and modem core lines up with where the wire will pass by guide wheels. Insert and tighten the socket head bolt through the hole in the center of the endcap. Ensure that the endcap faces seals the cylinder completely, and lightly grease and screw in the endplug in the center hole of the endcap.

Before the top endcap is attached, the CTD sensors and interface boards should be already mounted on the endcap. First, from the top end of the cylinder, attach the top chassis endplate (with large holes) to the two chassis tie rods inside the tube so that the threaded bolt in the center is accessible. Feed the CTD connector through a hole in the top endplate. Clean and grease the face seal o-ring (\#163) and place it in the groove on the endcap. The circular orientation of the endcap on the cylinder is not important. Plug in the CTD connector, and attach the endcap using a long 3/16" socket head driver through the attaching port on the endcap. Facing the endcap, the attaching port is the most counterclockwise hole covered by a plastic socket head plug. Ensure that the endcap faces seals the cylinder completely, and replace the socket head plug that seals the attaching port. 


\section{Appendix E: CTD configuration for PROCON}

The 41CP must be properly configured for the McLane PROCON software to acquire data from the CTD. When the sensor assembly first comes from the factory, several commands may need to be given to the unit to ensure that the 41CP does not cease profiling in air and does not echo scan numbers or pressure values during profiling. Several commands may have to be given to the $41 \mathrm{CP}$ once (and the settings will remain in memory). This may be done with PROCON powered, operating the MMP software, and with the 41CP endcap connected. From the main menu select " $<5>$ Bench Test," then select " $<1>$ CTD Communication." PROCON will respond:

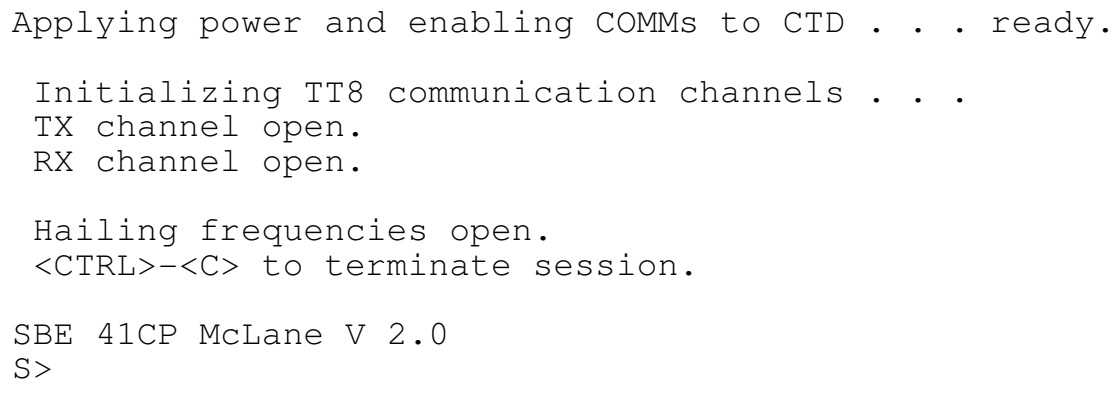

The last two lines are the wake up message from the 41CP. To ensure compatibility with the McLane PROCON software type:

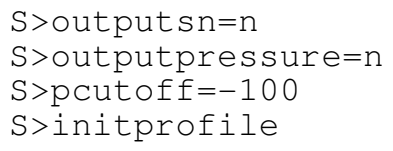

To determine the instrument status type:

$$
S>d s
$$

Here is a sample expected response:

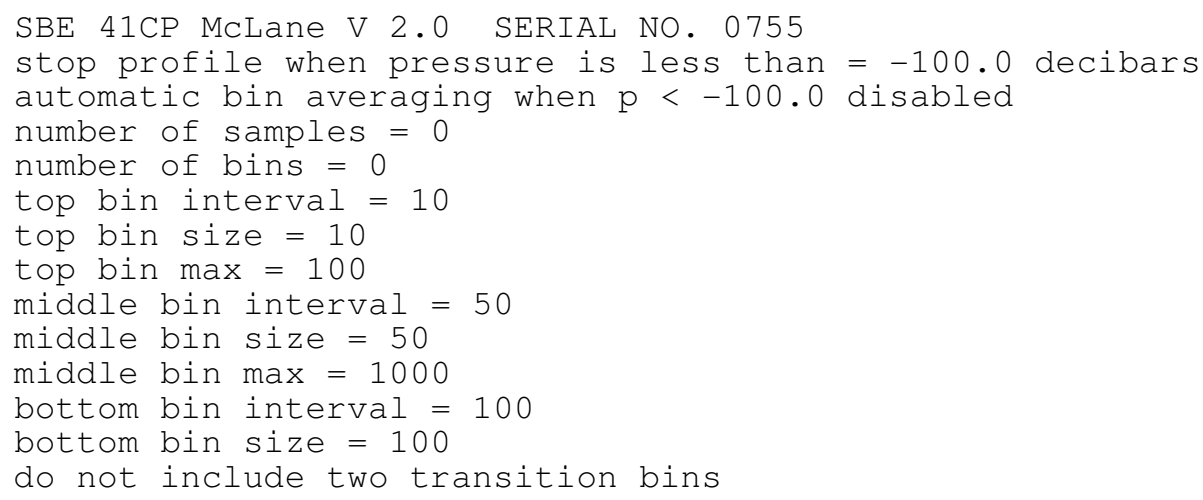

The calibration coefficients for the device may be listed, for example: 


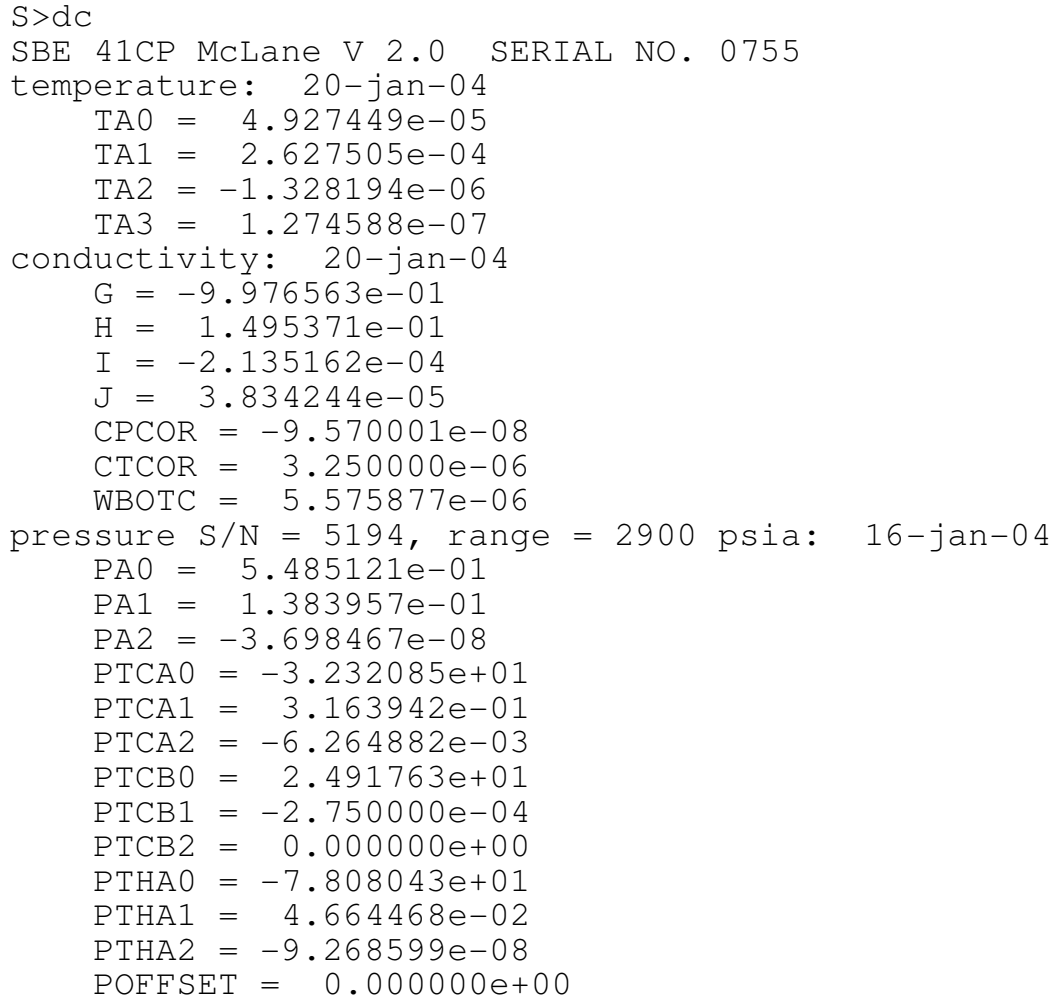




\section{Appendix F: Operating the profiler}

Applying Battery/Power

The power connector for the PROCON electronics expects two grounds and eight +10.8 voltages connections. When powering PROCON from a single two-wire power source, an adapter must be used which jumpers the two ground leads together and jumpers the eight power leads together.

A cable for ITP serial communications should be connected to the serial port of a computer and a terminal emulation program started ( 9600 baud, no parity, 8 data bits, 1 stop bit). The communications cable should be connected to the ITP after connecting the battery. Likely, the beginning of the startup message will be missed, and the first words received from the ITP will be the countdown seconds. It is important that the operator assert control over the software by typing <CTRL-C> within 30 seconds after applying power. Otherwise the independent watchdog timer will not be properly initialized and may reset the software schedule at a later date.

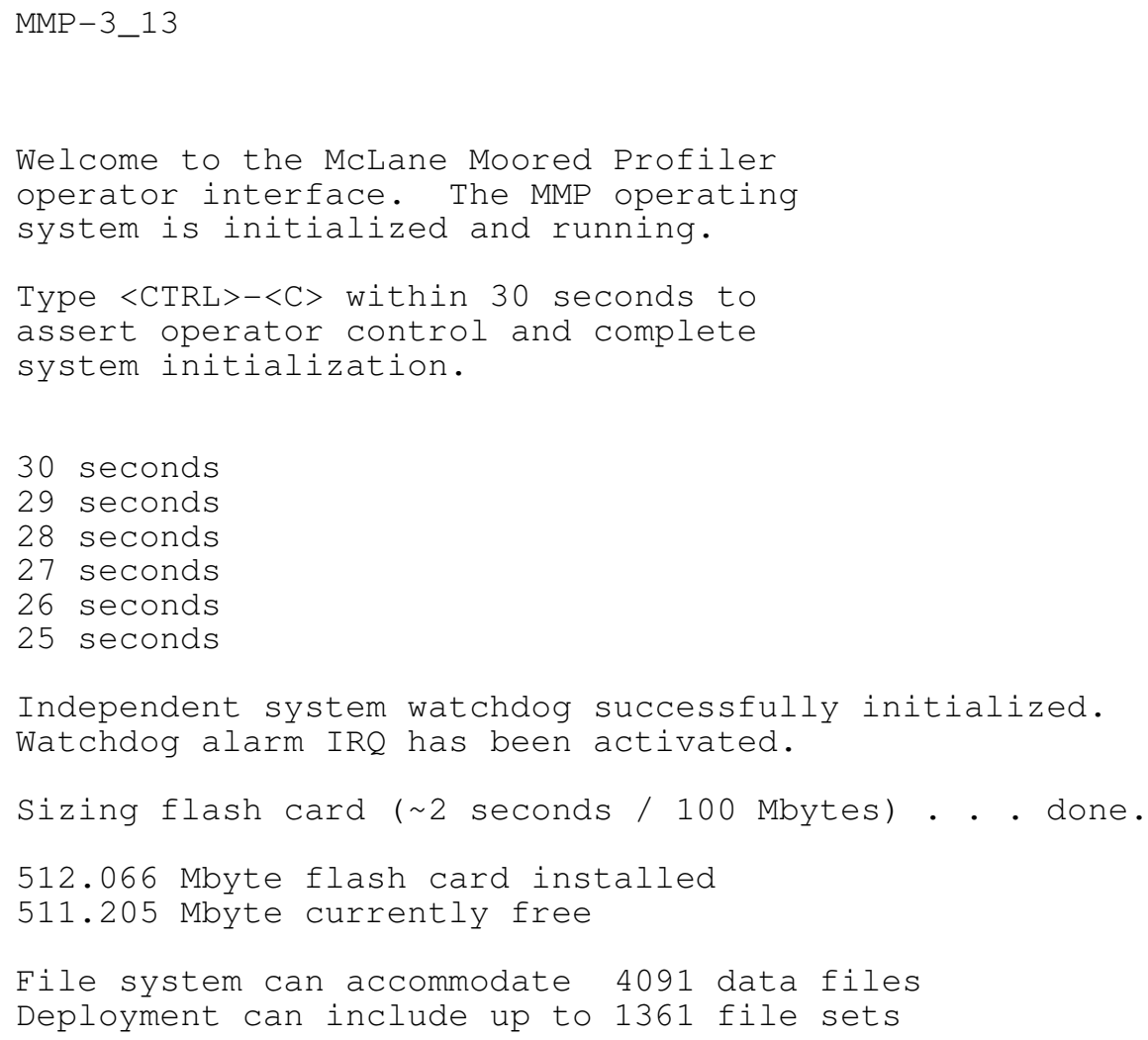

Set Time

Clock reads $06 / 23 / 2004 \quad 08: 52: 30$ 


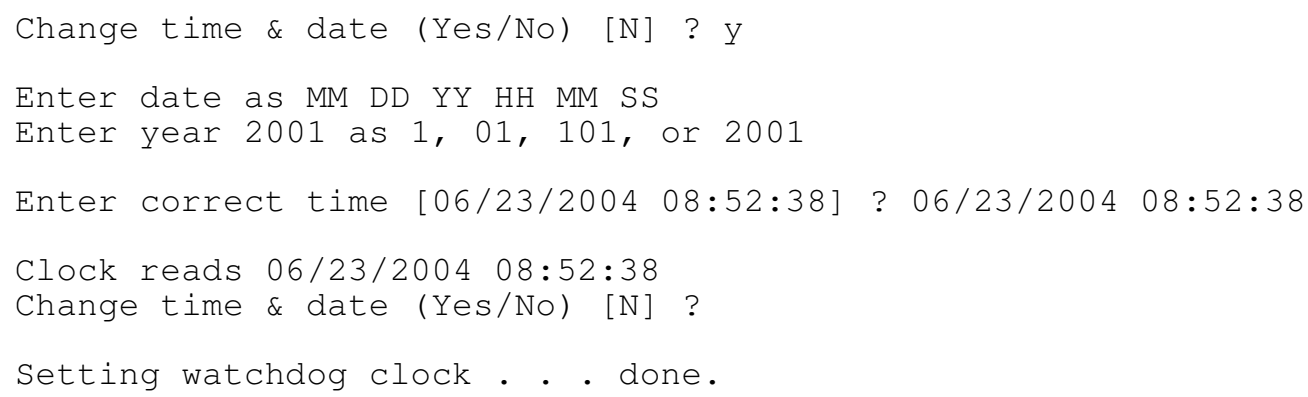

\section{Set Trip Meter}

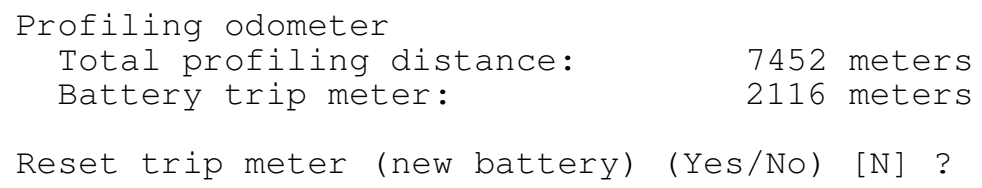

\section{Main Menu}

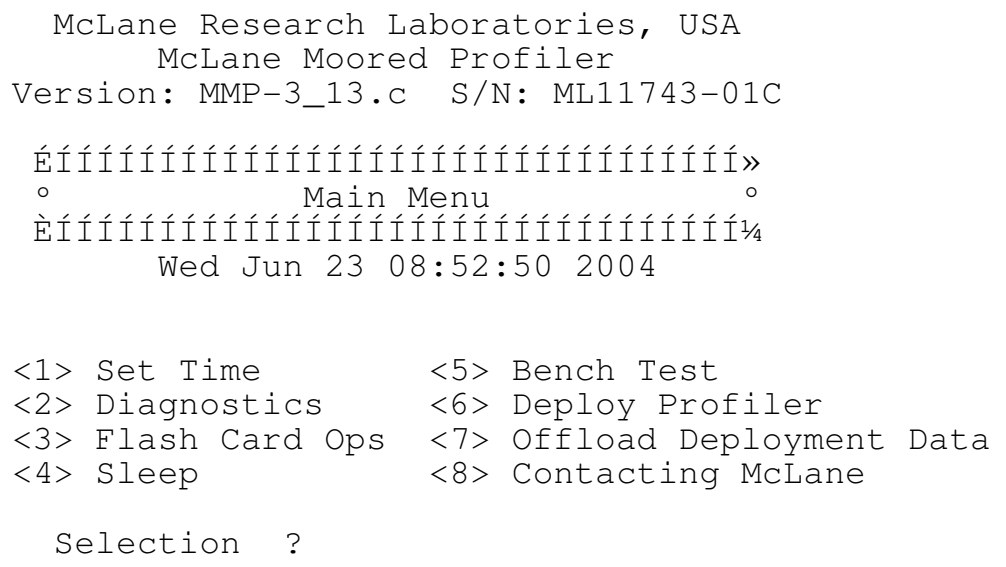

\section{Configuration Menu}

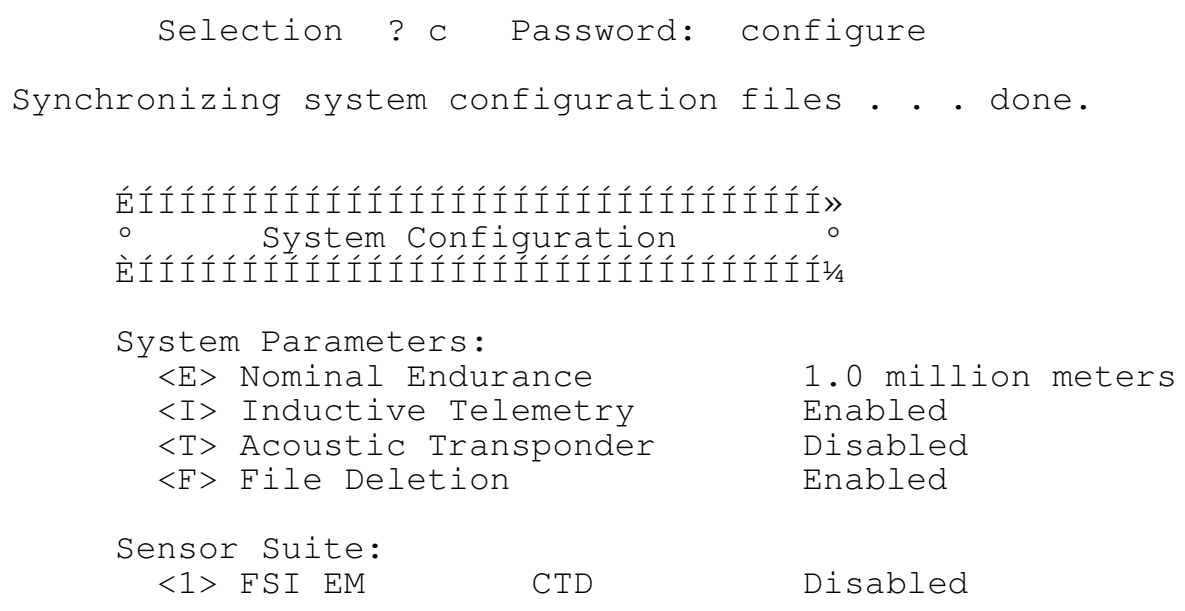




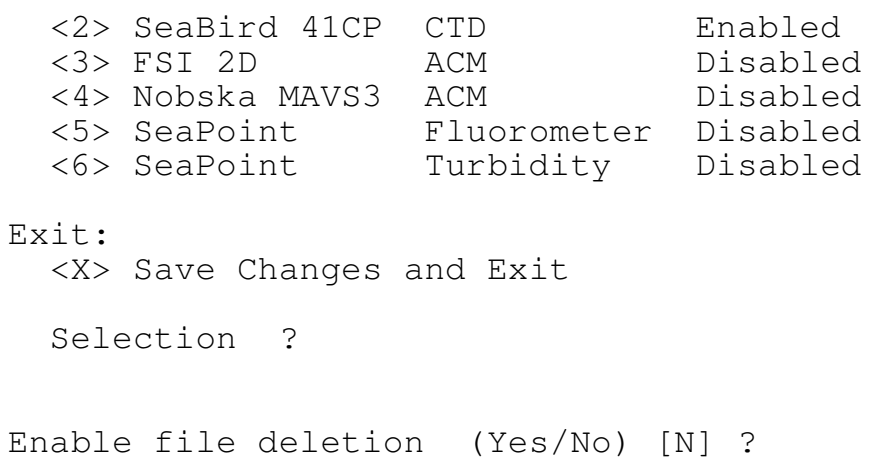

\section{Diagnostics}

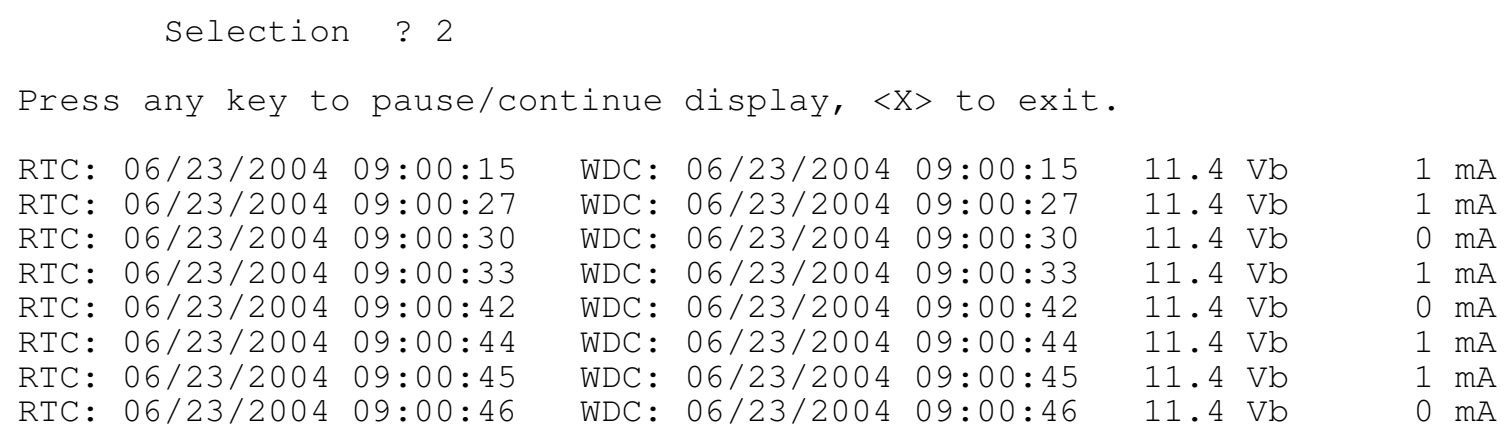

\section{Flash Card Operations}

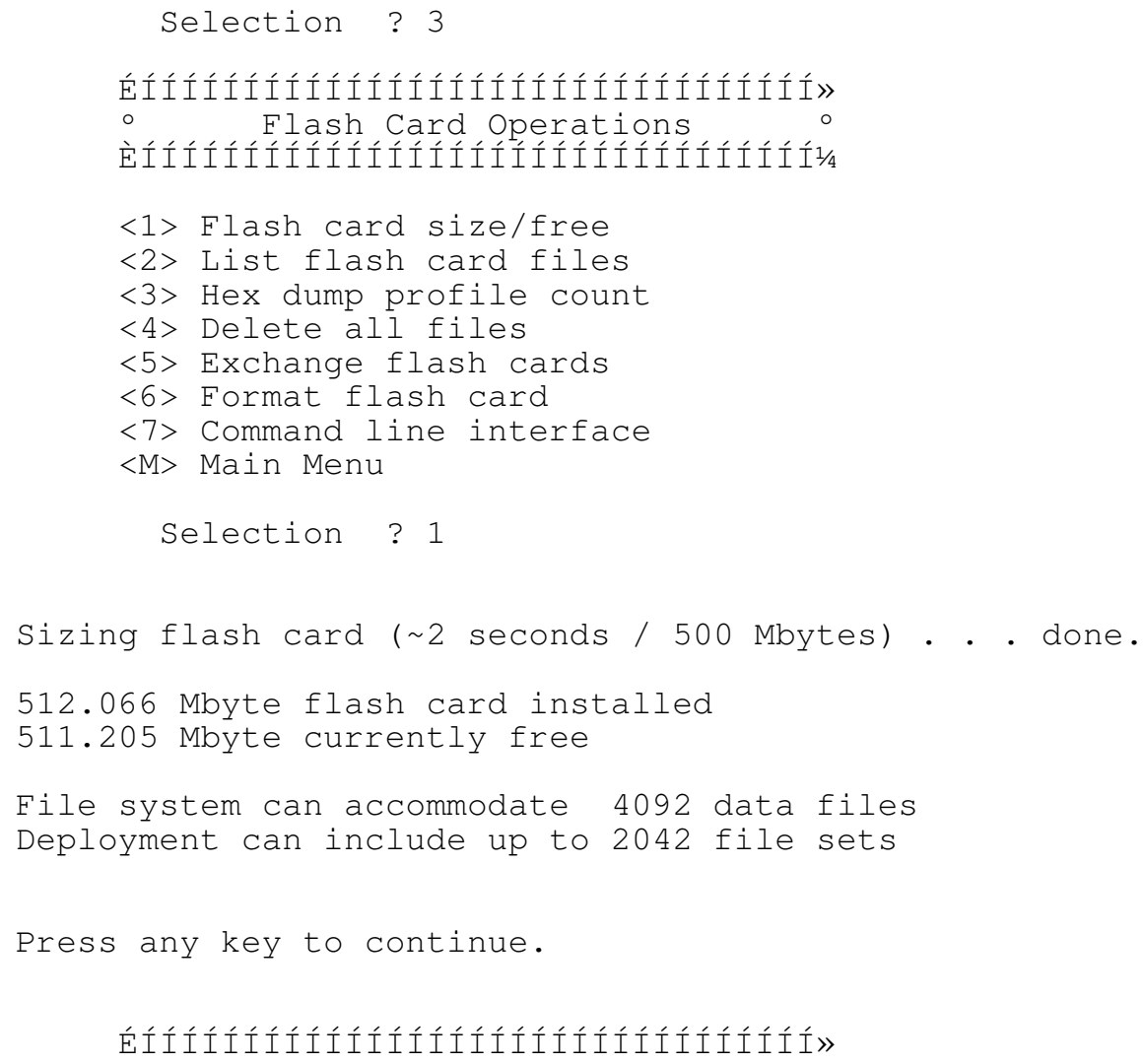




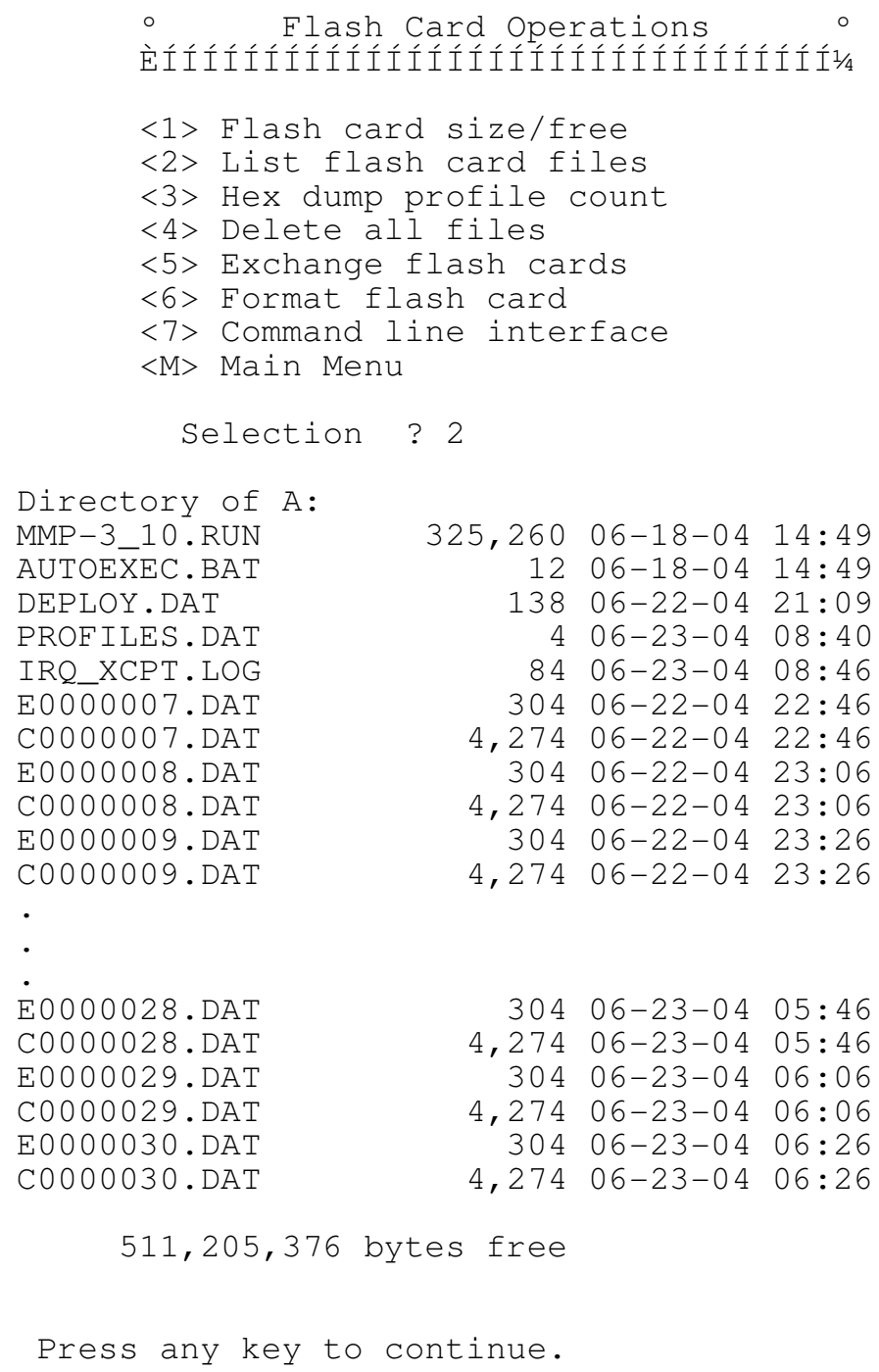

Command Line Interface (available for diagnostics, but not normally used)

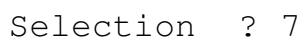

\begin{tabular}{|c|c|c|c|}
\hline$=====$ & PicoDos Intrinsic Commands & plus. RUN and & .BAT Files) \\
\hline COPY & source dest & DATE & (prompts) \\
\hline DEL & filename & DIR & [file | wildcards] \\
\hline ERASE & filename & FORMAT & {$[/ \mathrm{Q}][/ \mathrm{E}][/ \mathrm{F}]$} \\
\hline TIME & (prompts) & TYPE & filename \\
\hline REN & oldname newname & VER & (PicoDOS version) \\
\hline G & or GO [address ] & $\mathrm{LO}$ & {$[\mathrm{ofs}][; \mathrm{Bx}[+]] \quad[; \mathrm{G}]$} \\
\hline $\mathrm{MD}$ & [address ] & MM & [address ] \\
\hline$X S$ & {$[/ Q][/ X][/ C] f i l e$} & $\mathrm{XR}$ & {$[/ Q][/ X][/ C][f i l e]$} \\
\hline YS & [/Q] [/G]file[,file...] & YR & {$[/ \mathrm{Q}][/ \mathrm{G}]$} \\
\hline BAUD & [newrate] [/Q] & SAVE & file [start] [end] \\
\hline DUMP & file [start [, end] ] & CAPTURE & file[delim] [/N] \\
\hline $\mathrm{BOOT}$ & [PICO ] [ TOM8 ] & $\mathrm{CCC}$ & (card change) \\
\hline RESET & (hard reset) & TOM8 & (exit to TOM8) \\
\hline
\end{tabular}




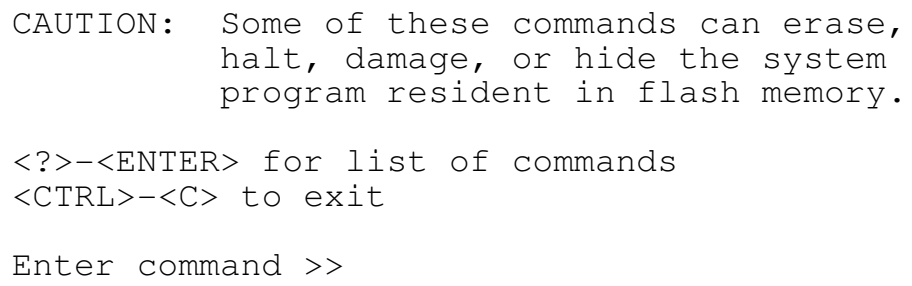

\section{Software Reboot (not normally used)}

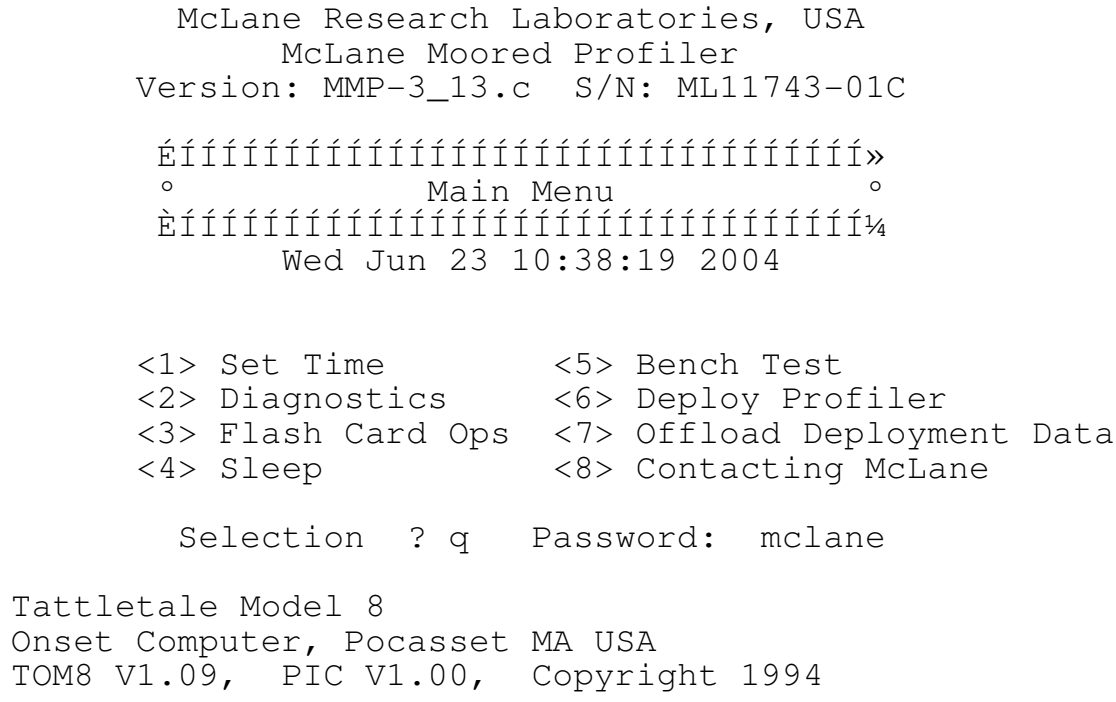

\section{Sleep Mode}

Selection ? 4

Verify sleeping mode current is approximately $180 \mathrm{uA}$. 
Bench Test

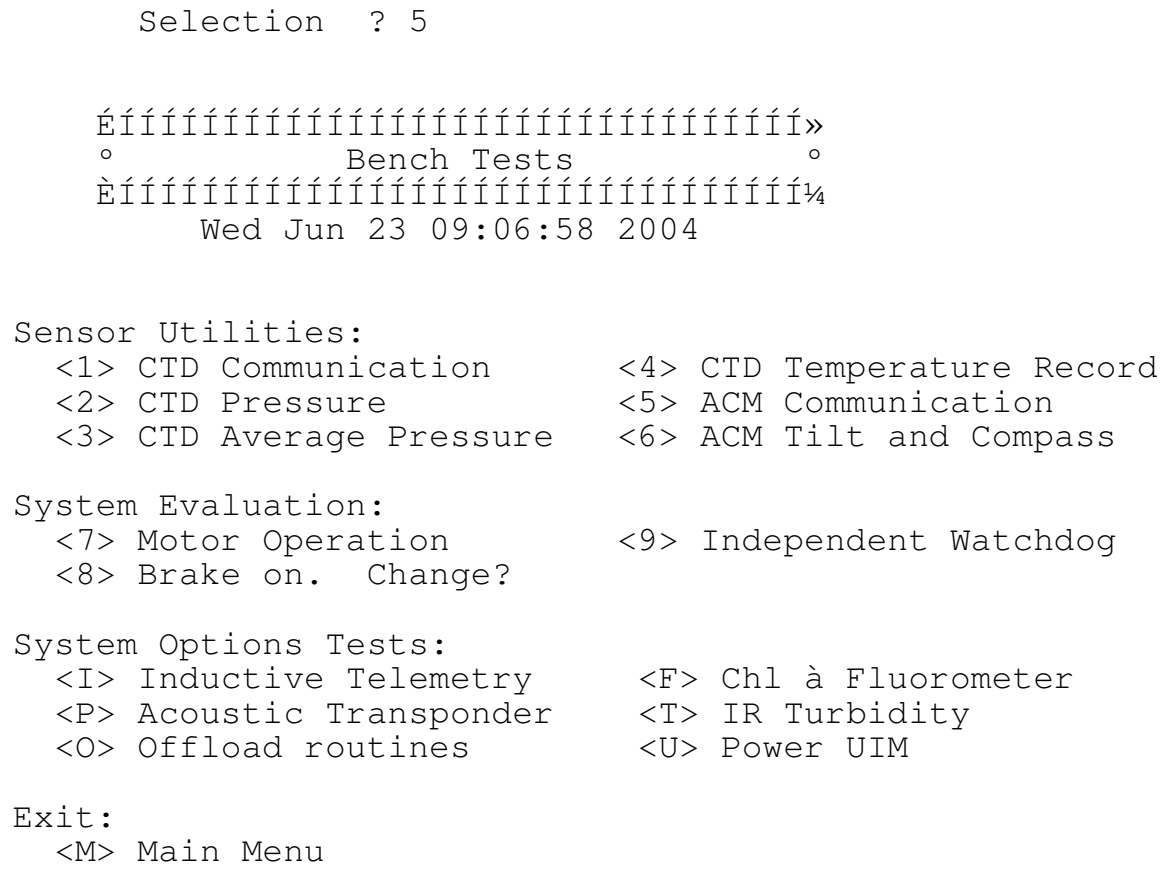

\section{CTD Communication}

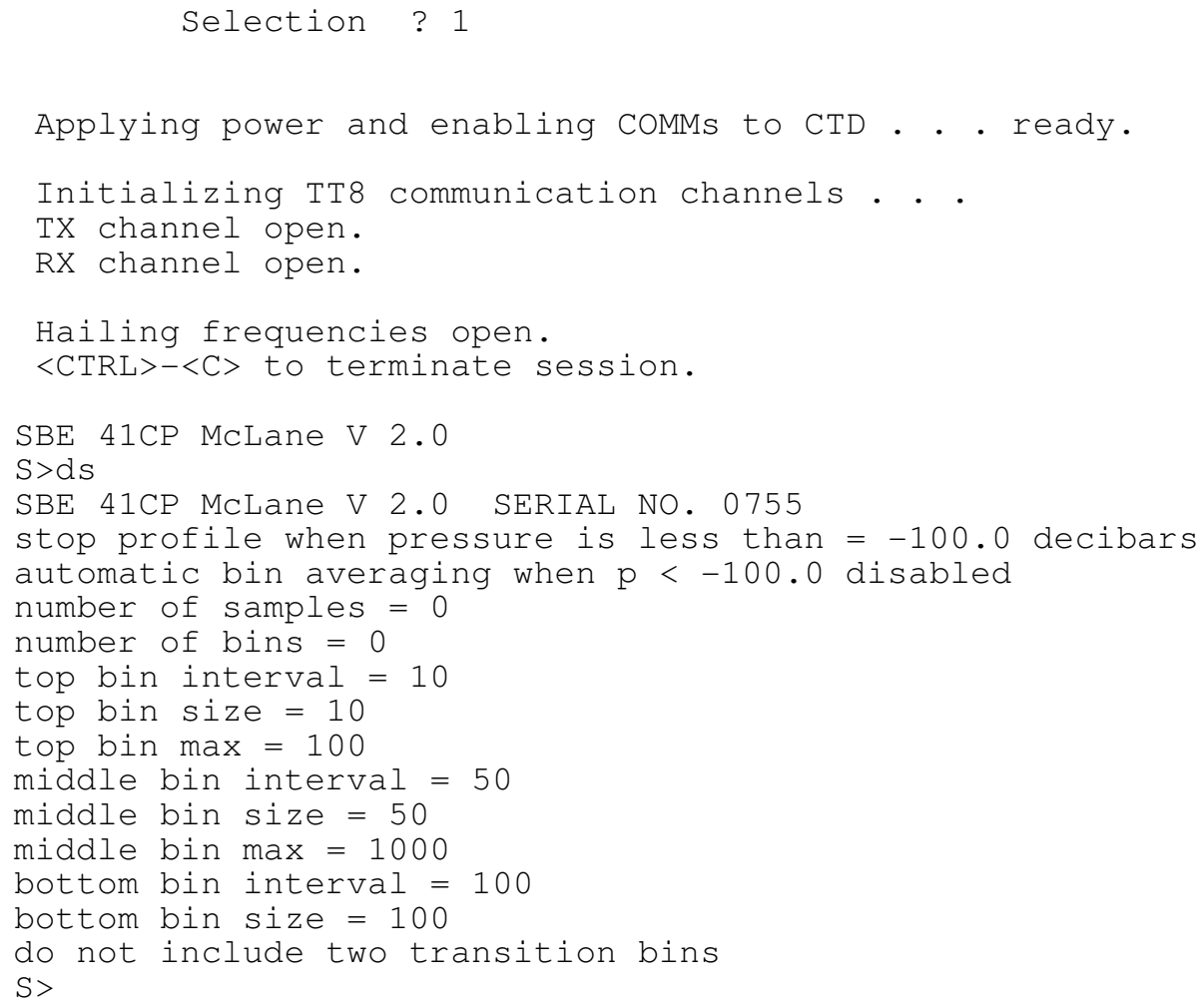


TX channel closed.

RX channel closed.

Shutting down power to CTD.

Press any key to continue.

Verify current is approximately $29 \mathrm{~mA}$. Ctrl-C to power off CTD

CTD Average Pressure

Selection ? 3

Verify $\mathrm{N}$ pressure measurements recorded.

\section{CTD Temperature}

Selection ? 4

Verify $\mathrm{N}$ temperature measurements recorded.

\section{Motor Verification}

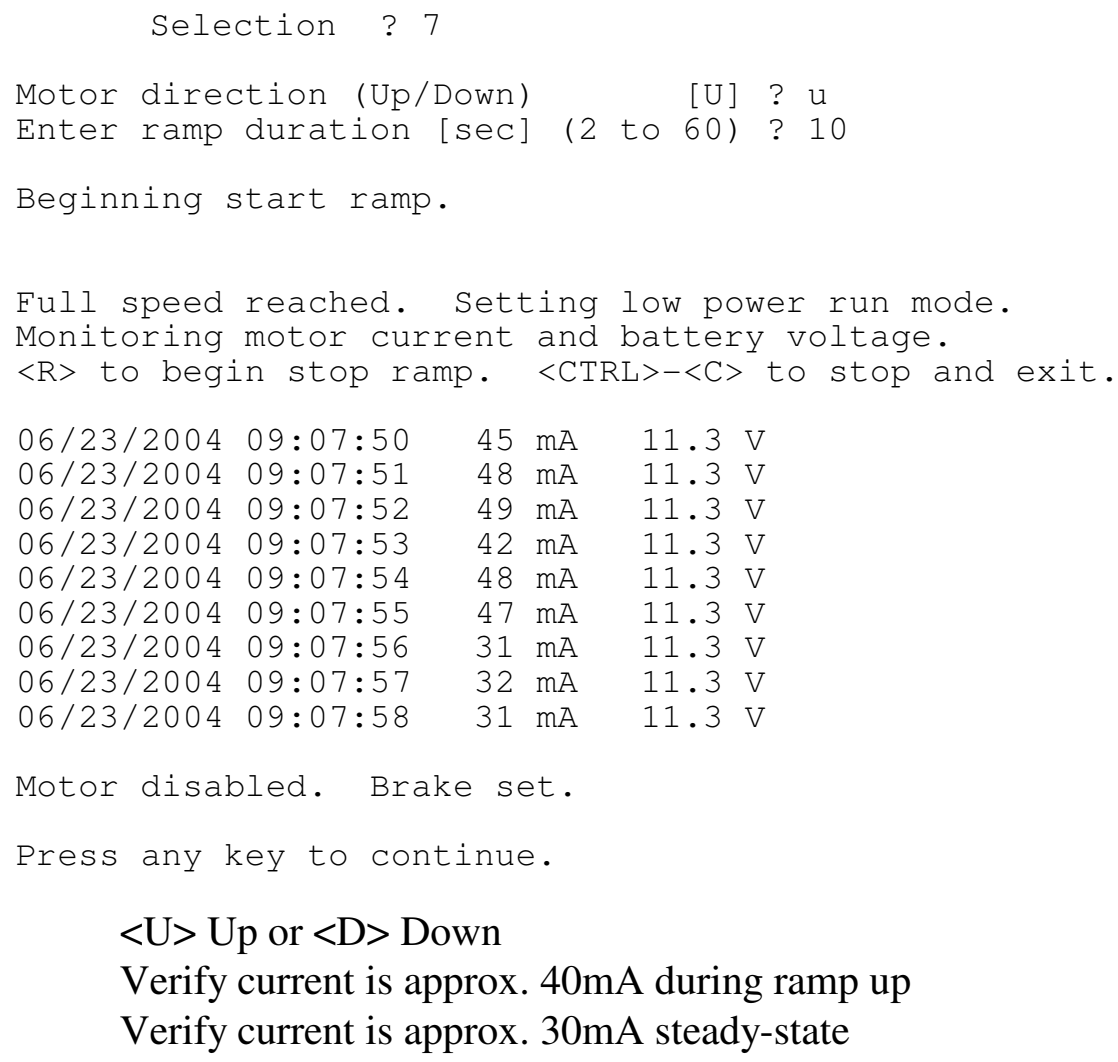


$<\mathrm{R}>$ to begin stop ramp

Verify current is approx. 40mA during stop ramp

Verify current is approx. 10mA at motor stop

Verify current returns to approx. $2.3 \mathrm{~mA}$

Brake test

Selection ? 8

Independent Watchdog

Selection ? 9

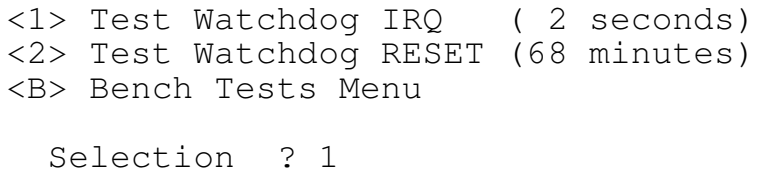

\section{UIM Verification}

Selection ? U

Verify current is approx. 14-15mA

Verify current is approx. 19mA when tone is present

Verify tone waveform is $4800 \mathrm{~Hz}$ (approx. $0.2 \mathrm{mS}$ period) and approx. $20 \mathrm{mVp}$-p

Verify tone waveform is on for approx. 3 seconds

Verify current is approx. 14-15mA after tone

Verify after 40 seconds from step 4 , current is approx. $2.5 \mathrm{~mA}$

Verify UIM is configured as follows:

口 SBE 44 UNDERWATER MODEM V 1.8

口 $\quad$ senor baud rate $=9600$ 
- break character length $=1000$ milliseconds

- time out after 30 seconds without receiving a valid command

- termination character is 62 , char $=>$

口 Relay Command Settings:

a relay termination characters $=\langle\mathrm{CR}\rangle\langle\mathrm{LF}\rangle$

- total time for response $=600$ seconds

a wait 0 milliseconds before sending the command

- halt relay after a gap of 5500 milliseconds between characters

- GDATA Command Settings:

a total time for response $=30$ seconds

a wait 0 milliseconds before sending the command

- halt acquisition after a gap of 1000 milliseconds between characters

- GDATA command string = NO STRING

- include gdata reply delay in datann reply

- do not enable control line on power up

- disable control line before relaying command

- disable control line before sending GDATA command

a do not switch power to sensor on power up

a disable switch power to sensor before relaying command

- disable switch power to sensor before sending GDATA command

口 send tone on powerup

Deployment procedure

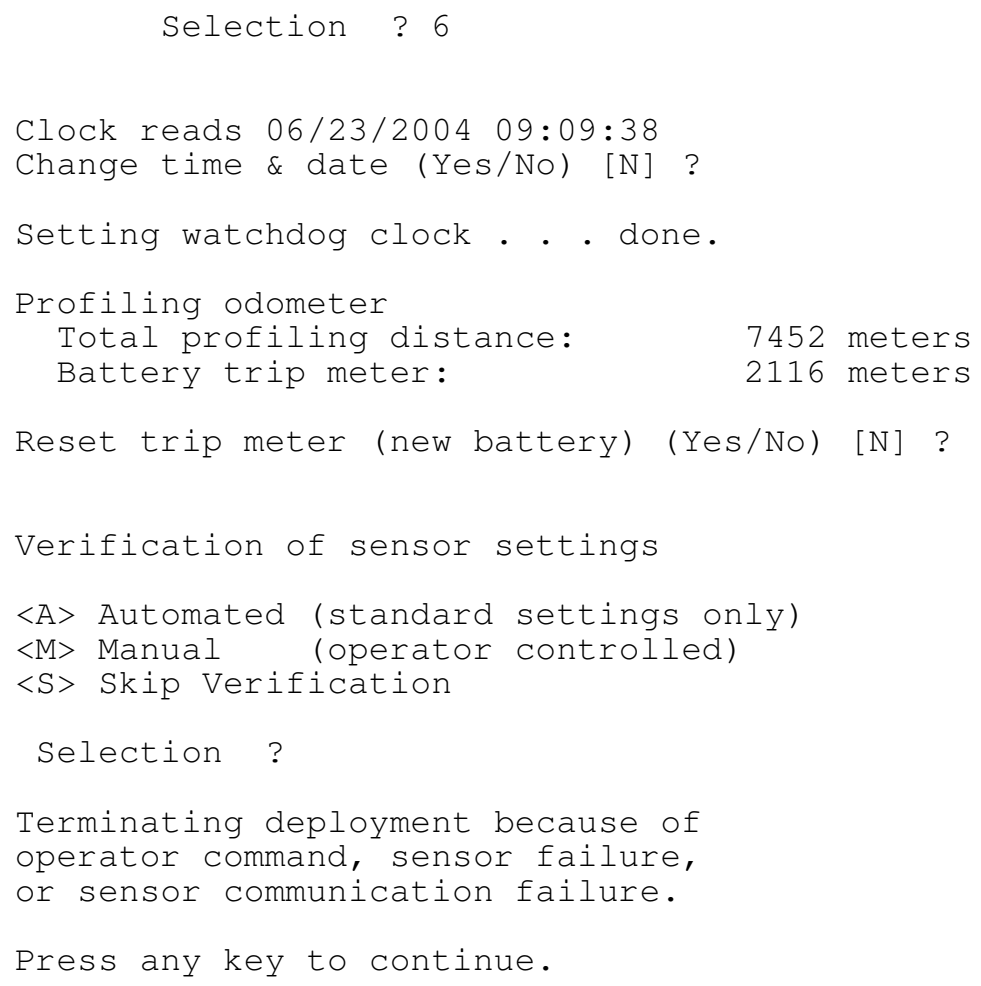




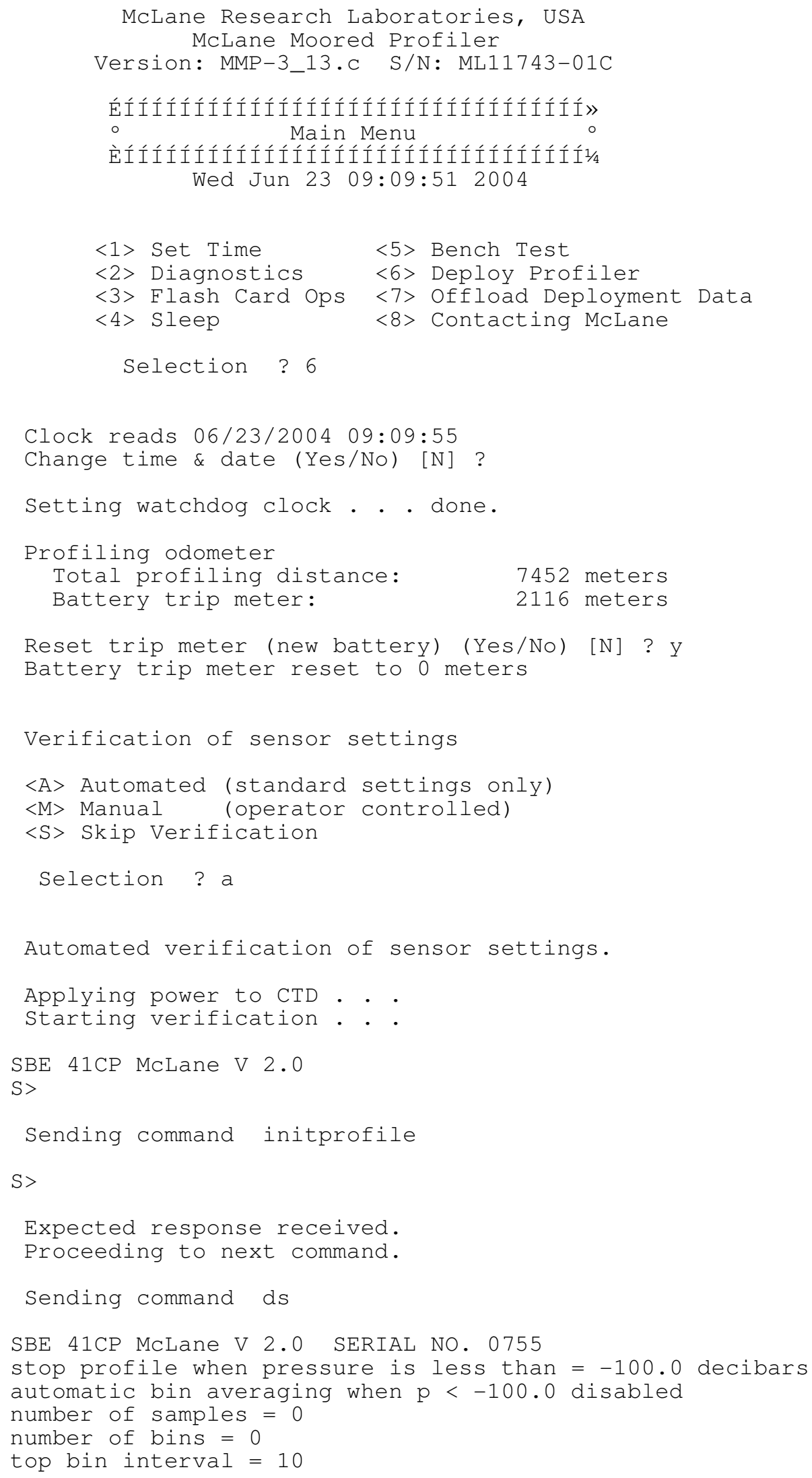




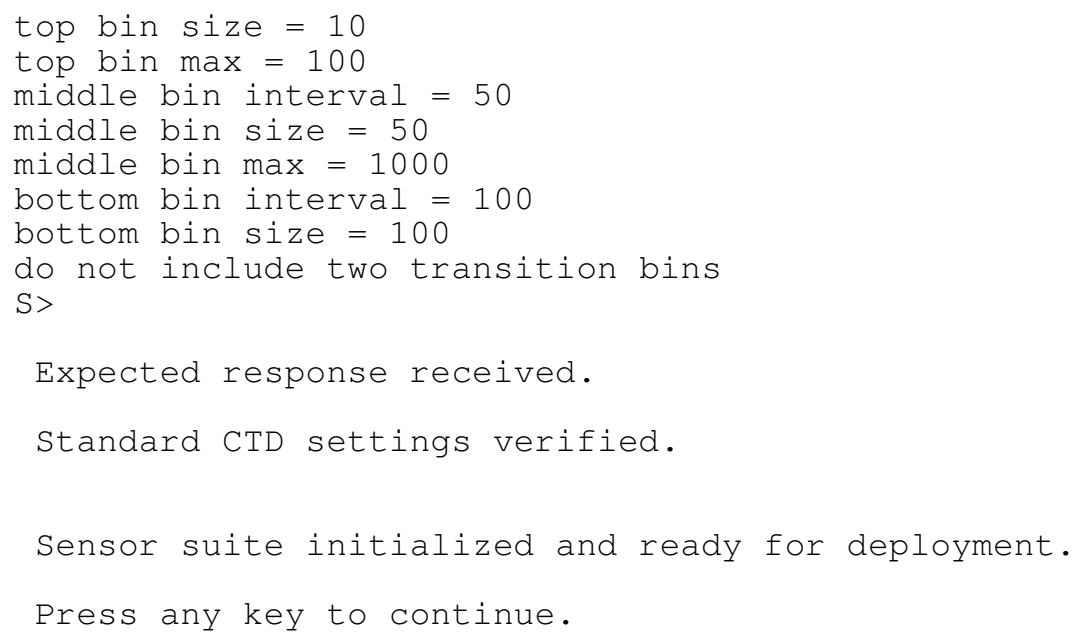

\section{Deployment Settings (example from ITP2)}

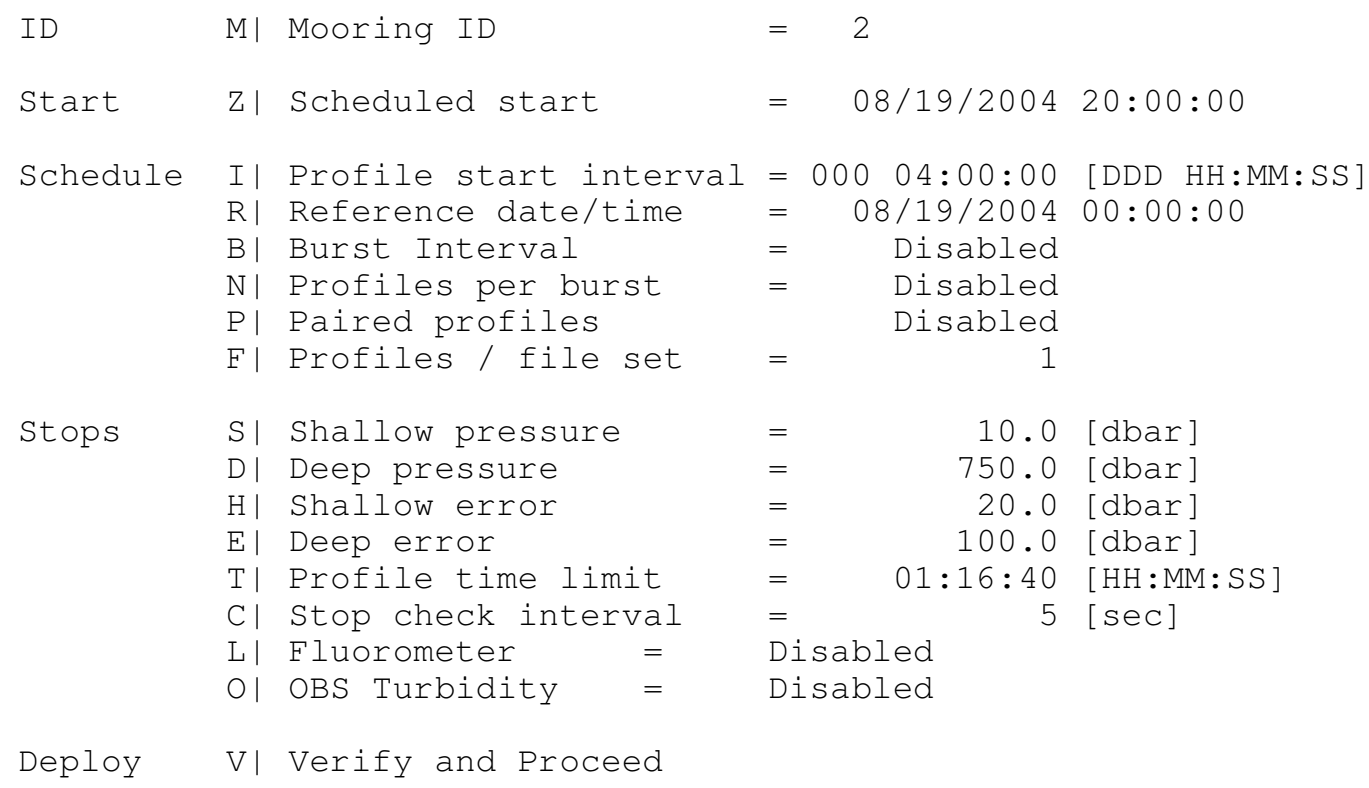

\section{Verify and Proceed}

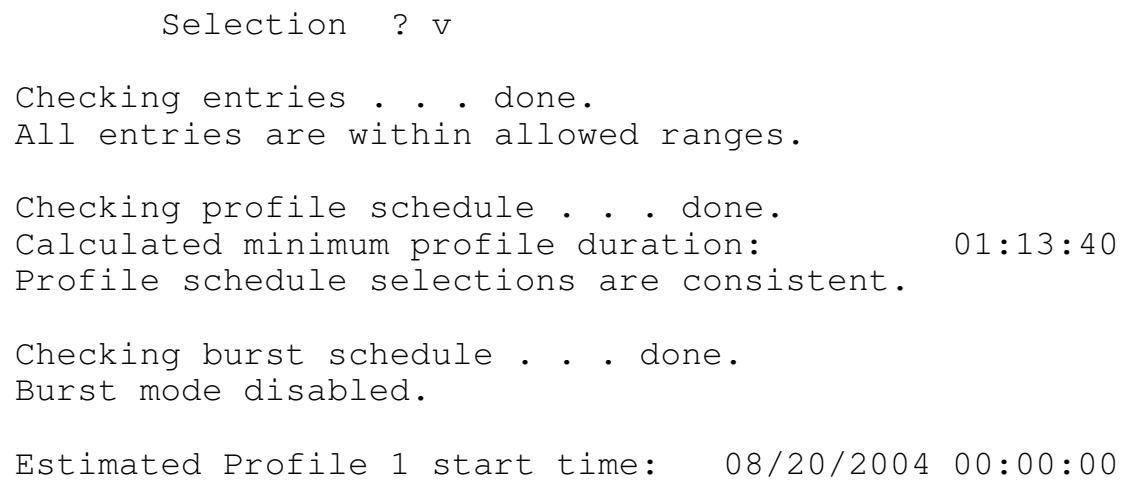




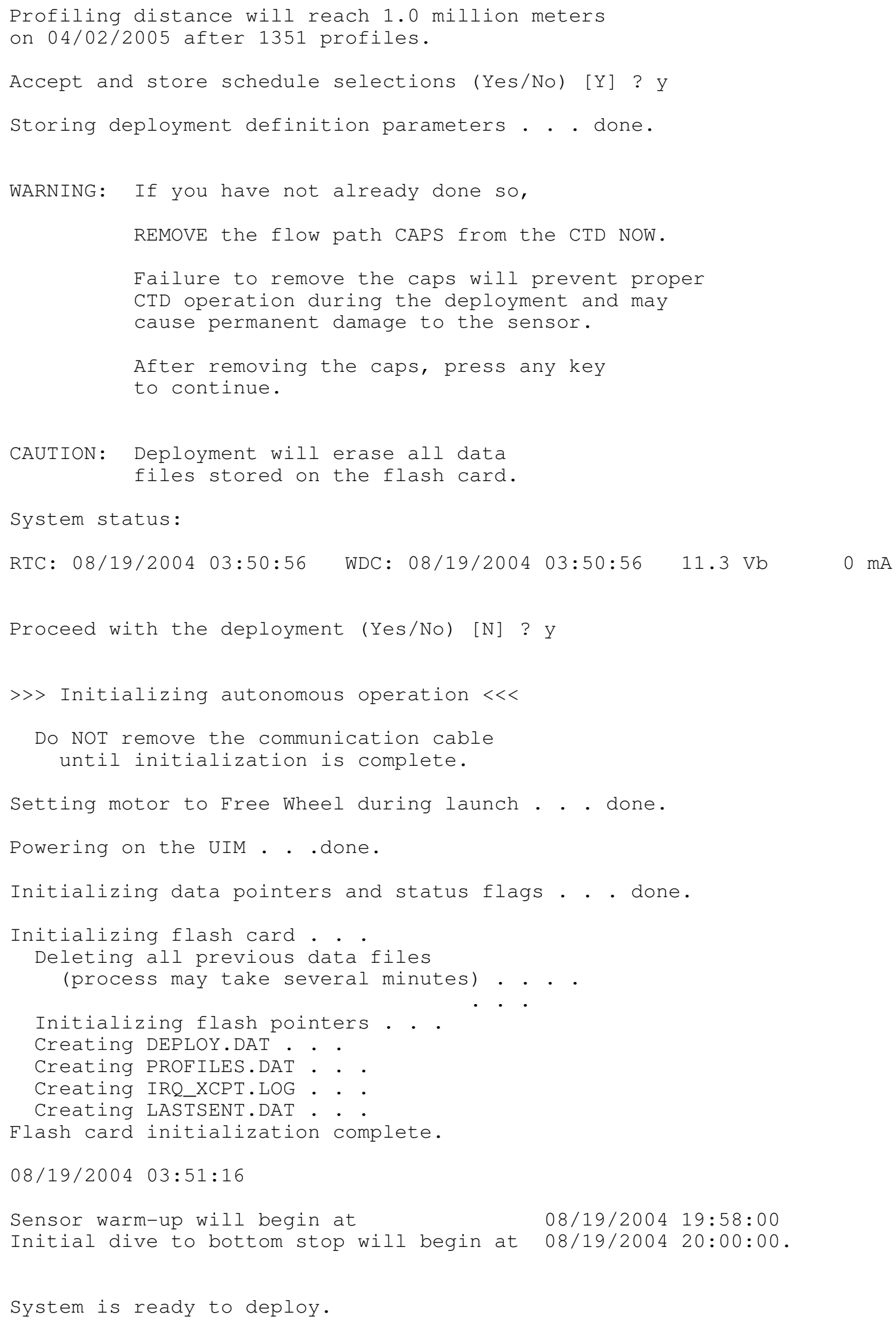


Remove communication cable Now, apply dummy

plug to communications port, and attach

faired bottom cap to vehicle.

08/19/2004 03:51:18 Sleeping. . .

\section{Recovery procedure}

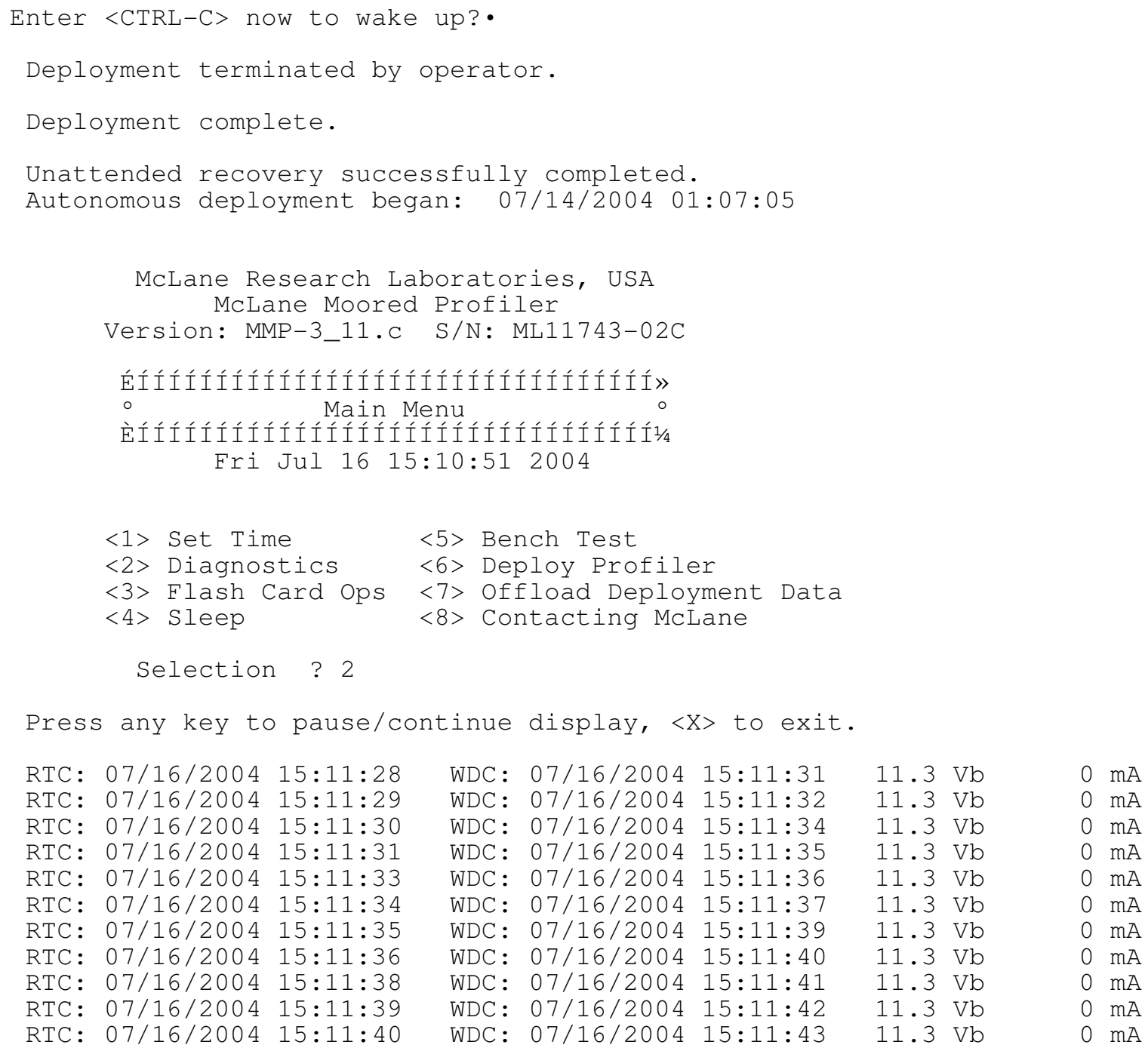

\section{Dump Log Files}

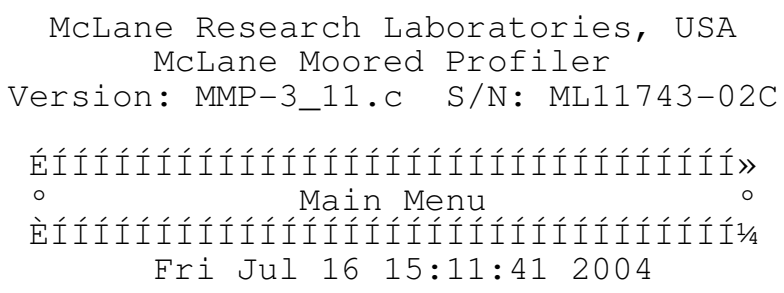




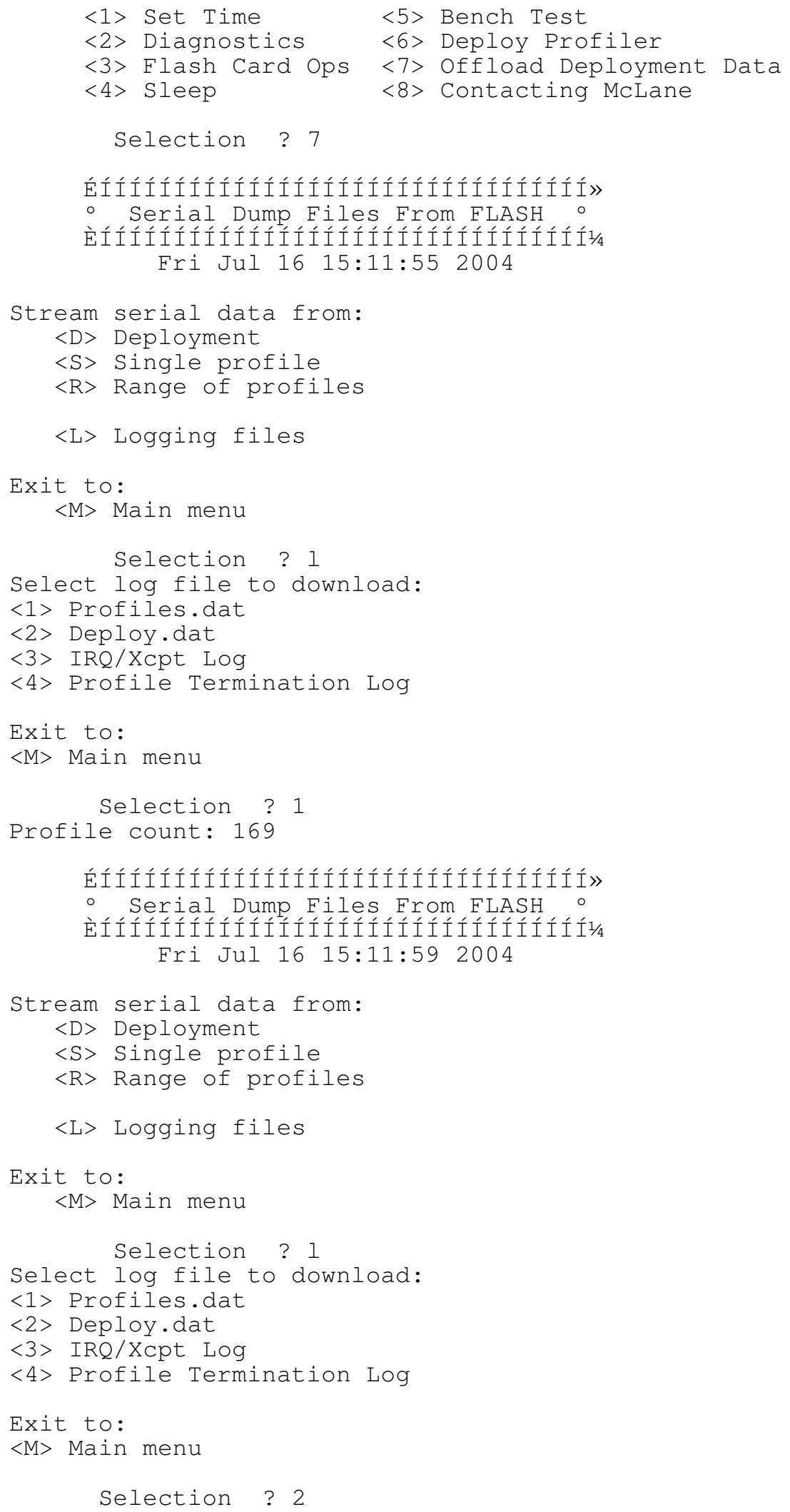


Countdown delay

Profile start interval

Reference date/time

Burst interval

Profiles per burst

Paired profiles

Profiles / file set

Shallow pressure

Deep pressure

Shallow error

Deep error

Profile time limit

Stop check interval

Transmission duration

SYSTEM CONFIGURATION

Nominal Endurance

Inductive Telemetry

Acoustic Transponder

FSI EM

CTD

SeaBird 41CP CTD

FSI 2D ACM

Nobska MAVS3 ACM

SeaPoint Fluorometer

Seapoint Turbidity

File Deletion

INTERNAL PARAMETERS

Fullspeed

PR_Threshold

PR_TimeThreshold

sensor_warmup

sensor_warmdown

InfiniteDeployment
$00: 30: 00 \quad[\mathrm{HH}: \mathrm{MM}: \mathrm{SS}]$

$000 \quad 01: 00: 00$ [DDD HH:MM:SS ]

07/01/2004 00:00:00

010 00:00:00 [DDD HH:MM:SS ]

Disabled

Disabled

3.0 [dbar $]$

13.0 [dbar]

2.0 [dbar]

3.0 [dbar $]$

$00: 25: 00 \quad[\mathrm{HH}: \mathrm{MM}: \mathrm{SS}]$

1 [sec]

15 [min]
1.0 million meters

Enabled

Disabled

Disabled

Enabled

Disabled

Disabled

Disabled

Disabled

Disabled

( IR)

with profiles stored $=30$

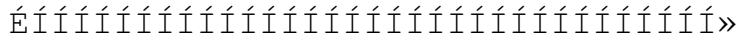




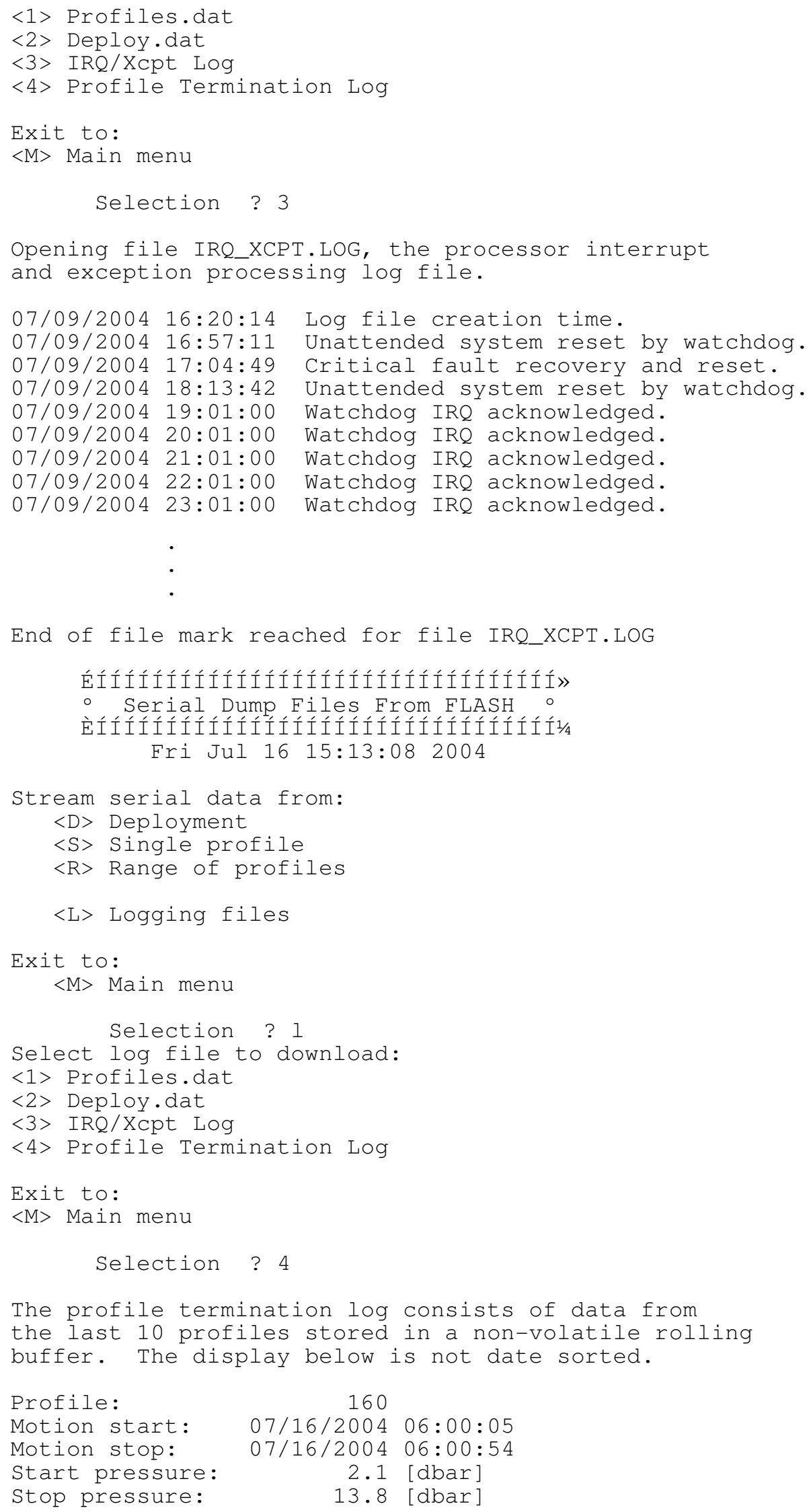




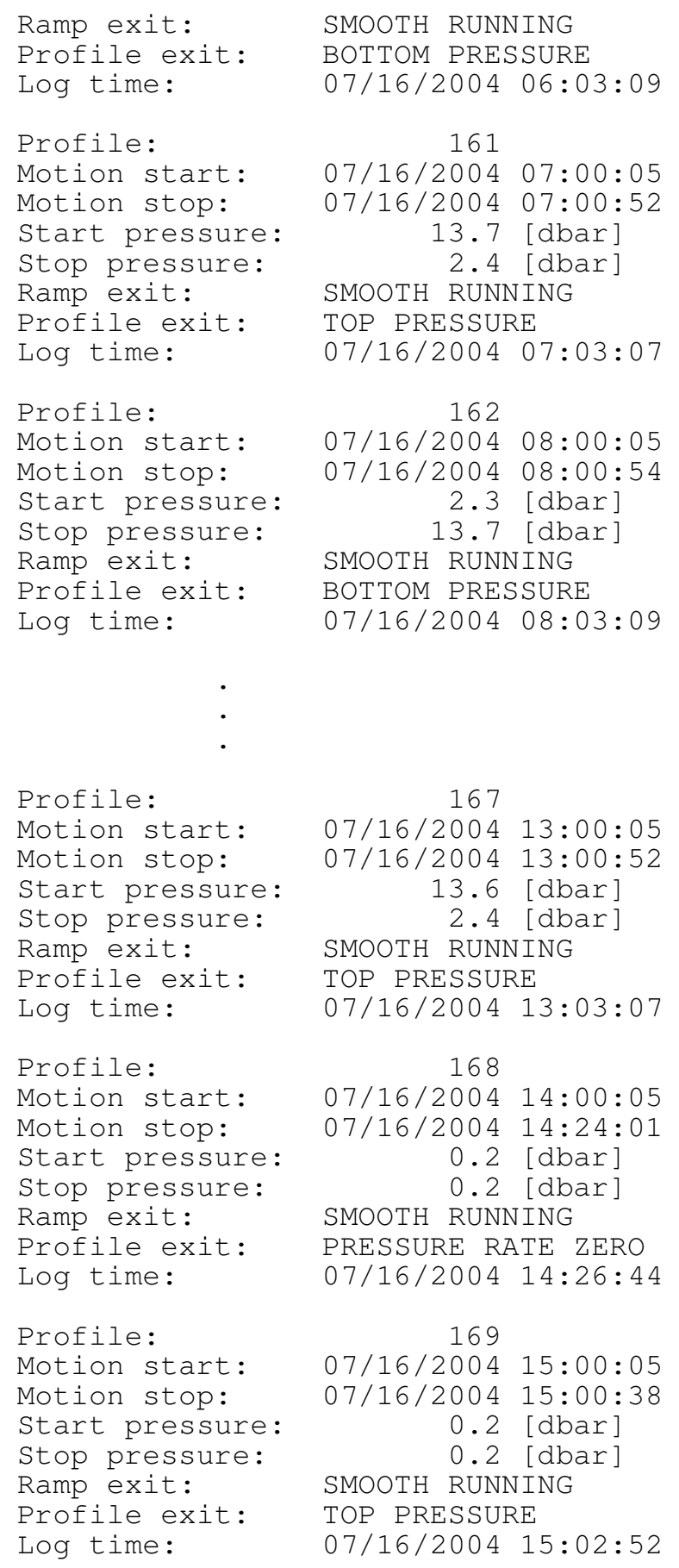




\section{Appendix G: IM Protocol}

The order of events is: PROCON acquires data $->$ powers UIM $->$ awakens TD $->$ awakens SURFCON-> SURFCON powers SIM. Thereafter SURFCON prompts PROCON, subject to timeouts at either end, to transfer the information using 3 requests:

1) normally, REQNEW, to request a new file, presumably type *.DAT

2) REQFIL to request a specific file

3) REQDIR to request a limited directory of available files

The transmission protocol for 3 is that the directory is sent as a sequence of entries without delimiting characters. The protocols for the other two file types are more rigorous. In all cases SURFCON first sends a request packet, and PROCON responds first with a 50-character ASCII metadata packet. An example of the metastructure is ('*' represents a 0x00 and a " ، represents a space $0 \times 20)$ :

E0000000.DAT*mm dd yyyy hh mm ss $\operatorname{ccc}^{*} \operatorname{xxxxxxxxxxx^{*}>}$

SURFCON then follows with a sequence of binary packets of length 4096 bytes. After receiving each packet, SURFCON responds with an "ACK" confirming proper reception, or "NAK" confirming errors and which will invoke up to 3 re-transmissions. After SURFCON gets a packet correctly from the PROCON, it strips off the header bytes and appends the current file. Each *.DAT or *.ENG file will be reassembled in SURFCON, and an associated metadata file will be flagged according to a CRC test. Approximately 50 Kbytes of data are transmitted per profile. In all cases, SURFCON expects that the PROCON UIM id $=01$, which is used below in the description of operations. After receiving an entire file, the SURFCON metastructure which consists of the PROCON metadata and the file CRC, is appended:

E0000000.DAT*mm dd yyyy hh mm ss ccc*xxxxxxxxxxx*000000ff 00000000000

If a filename does not exist then the PROCON metadata structure will begin with ENDOFDAT.DAT.

The detailed sequence for protocols 1 and 2 is:

- Upon wakeup by PROCON, SURFCON normally requests a new file by sending the command \#idREQNEW <CRLF>, it expects ASCII metadata (with 10 second timeout) if a new profile exists, else the metadata filename is "EOD". [“id" is always UIM number " 01 "]

Or, if SURFCON sends \#idREQFIL filename.ext $<$ CRLF> (including white space between \#idREQFIL and filename.ext), it expects ASCII metadata (with 10 second timeout) for that specific file. If PROCON does not have the file, it sends filename "EOD" in the metadata. 
[Example if file exists: "filename.ext mm dd yyyy hh mm ss mid xxxxxxxxxxx >"

Example if file does not exist: ENDOFDAT.DAT mm dd yyyy hh mm ss mid $\quad 0>$ ]

- If SURFCON timeouts waiting for a REQNEW or REQFIL response 3 times, it returns to suspend mode.

- If SURFCON receives reasonable metadata, it sends bidREQACK $<\mathrm{CRLF}>$ (where " $b$ " stands for binary transmission and "id" = "01") and expects a sequence of 1 or more binary packets, with header and up to 4096 bytes of data.

[The PROCON data packet header is defined as 4 ASCII characters "DAT_" 16 bit binary length count in bytes of this packet, little endian (low byte first), and 16 bit binary CRC $<$ CRLF> (little endian).]

- If SURFCON receives data packet correctly, it sends bidREQACK $<$ CRLF $>$ and expects the next data packet, etc.

- If SURFCON receives packet with errors, or times out, it sends bidREQNAK<CRLF> and expects repeat of the previous packet.

- If SURFCON receives the same packet with errors 3 times in sequence, it re-requests same file specifically, \#idREQFIL filename.ext.

- If SURFCON receives the PROCON CRC file terminating packet, it responds with bidREQACK $<\mathrm{CRLF}>$ and closes the file.

[The PROCON CRC packet is defined as 4 ASCII characters "CRC_" 16 bit binary length count in bytes of this packet, little endian (low byte first), and 16 bit binary CRC $<$ CRLF $>$ (little endian).]

The detailed sequence for protocol 3 is:

- If SURFCON sends \#idREQDIR<CRLF>, it expects an ASCII response, listing files PROCON has available in 23 byte entities each null terminated (example: filename.xxxnnnnnnnnnnn 10 ), each directory entry terminated with $<\mathrm{CRLF}>$.

Finally, SURFCON sends \#REQEOD $<$ CRLF $>$ to terminate the session with PROCON, ignores anything beyond this and suspends. No PROCON response is expected.

At the UIM/SIM level, the ASCII RELAY command termination character for the SIM (that causes it to switch from $\mathrm{Tx}$ to $\mathrm{Rx}$ is $\langle\mathrm{CR}>, 0 \mathrm{x} 0 \mathrm{~d}$; it ignores $<\mathrm{LF}>, 0 \mathrm{x} 0 \mathrm{a}$. The UIM switches from $\mathrm{Rx}$ to $\mathrm{Tx}$ for ASCII RELAY on the same $<\mathrm{CR}>$. The UIM knows that an ASCII RELAY response is done by reception of a ' $>$ ' from the PROCON, which can be set to a different default character. The BINARY RELAY delay for the UIM should be longer than for the SIM which 
means that the SIM must wait for the UIM turnaround delay before issuing the next relay request. This delay difference means that the SIM is never listening to an open line which would result in garbage characters when neither the SIM nor UIM are driving the loop. The relay length from PROCON to UIM and on must be less than $30 \mathrm{kbytes,} \mathrm{due} \mathrm{to} \mathrm{a} \mathrm{buffer} \mathrm{limit.} \mathrm{The} \mathrm{maximum} \mathrm{relay}$ command length on both SIM and UIM is 128 chars, including everything but the terminating $<\mathrm{CR}>$. Responses to SIM ASCII RELAY command by the UIM are terminated with ' $\$$ ' installed by the UIM. The reliability of the IM link is good so errors should be few. Care needs to be taken if testing with a long cable on a reel as the TD function may not work as well. The average inductive transmission rate of a long file between PROCON and SURFCON is about 70 characters/s over the 1200 baud link

A session overview for data transfer from ITP PROCON to SURFCON:

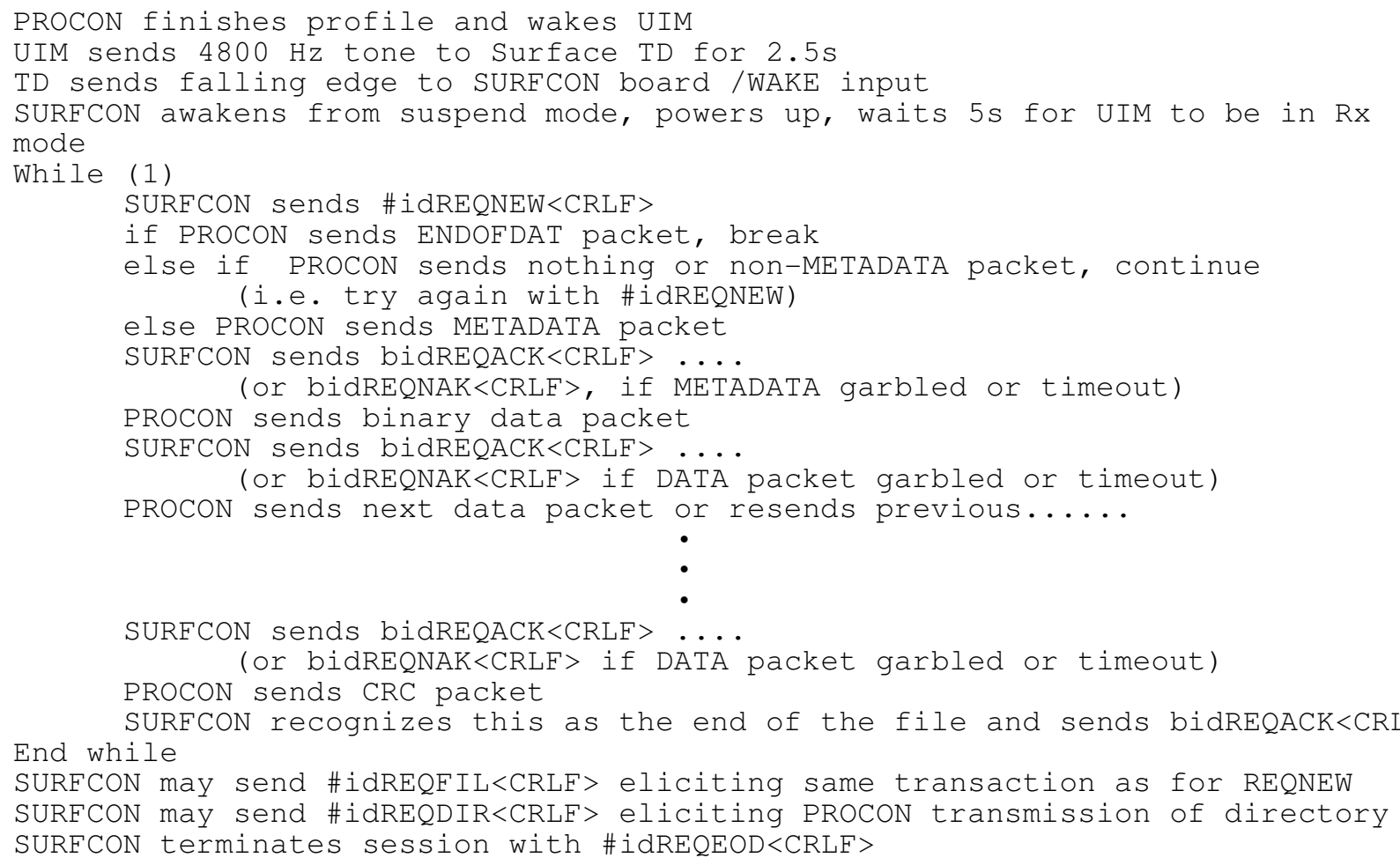

An example of data transfer from ITP PROCON, excerpted from SURFCON screen log:

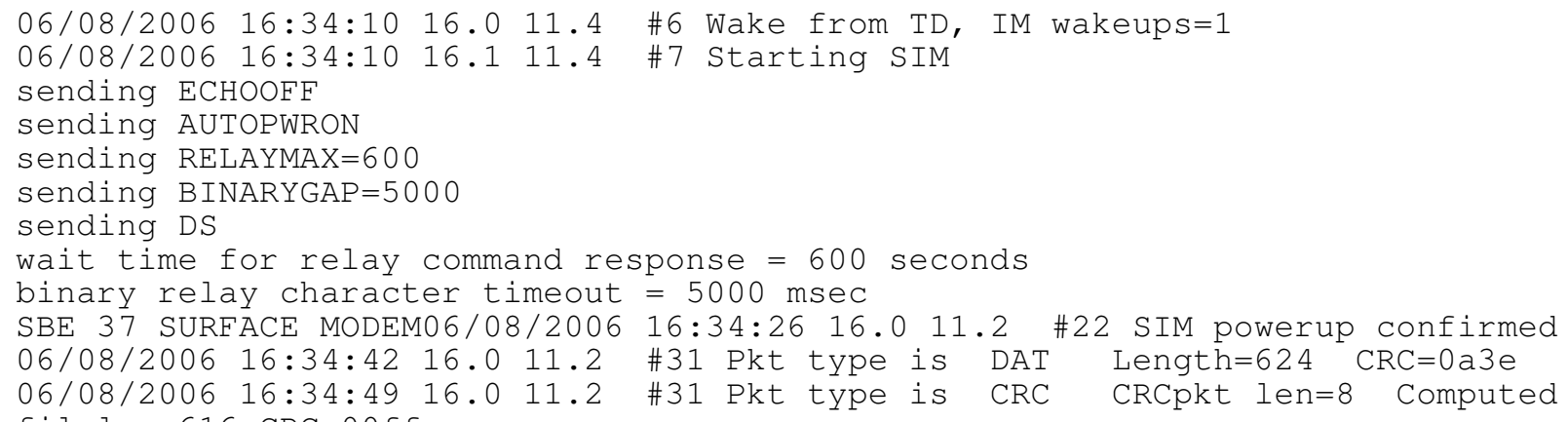


06/08/2006 16:34:49 16.0 11.2 \#10 Added file c:\DATA \E0000002.DAT to FileList 06/08/2006 16:34:49 16.0 11.2 \#33 IMFiletime after pkt loop = 21 sec, Total

IM time $=39$

06/08/2006 16:34:49 16.0 11.2 \#8 Sending REQNEW

C0000002.DAT $06082006143046001 \quad 2560>$

len of Meta response $=50$ sizeof metastruct $=50$

\#parms parsed=9 datafilename=c: \DATA \C0000002.DAT

06/08/2006 16:34:51 16.0 11.2 \#30 Openfile c:\DATA \C0000002.DAT for write

$06 / 08 / 200616: 35: 28$ 16.0 11.2 \#31 Pkt type is DAT Length=2568 CRC=06b4

06/08/2006 16:35:35 16.0 11.2 \#31 Pkt type is CRC CRCpkt len=8 Computed

filelen $=2560 \quad \mathrm{CRC}=00 \mathrm{ff}$

06/08/2006 16:35:35 16.0 11.2 \#10 Added file c: \DATA \C0000002.DAT to FileList

06/08/2006 16:35:35 16.0 11.2 \#33 IMFiletime after pkt loop = 43 sec, Total

IM time $=85$

$06 / 08 / 2006$ 16:35:35 16.0 11.2 \#8 Sending REQNEW

ENDOFDAT.DAT $06082006143516001 \quad 0>$

06/08/2006 16:35:40 16.0 11.2 \#11 Sending REQEOD

06/08/2006 16:35:43 16.0 11.3 \#34 IM time after task 1oop = 93 sec

06/08/2006 16:35:45 16.0 11.4 \#35 IM time after SIM 1oop = 95 sec

Q_CHECK nte $=1149795720$ now $=1149784545$

$0 \overline{6} / 08 / 2006$ 16:35:45 15.9 11.4 \#70 GetTimeToNextStatusEpoch: now =1149784545

interval $=3600$ tn $=1149786000 \mathrm{ntt}=0$

06/08/2006 16:35:45 15.9 11.4 \#18 suspend for 1455 s, next status 1455 s,

next ISU Tx $11175 \mathrm{~s}$ 


\section{Appendix H: SURFCON to Logger Communications}

Two logger computers running the Linux operating system have been established at WHOI to receive data via the Iridium global phone system from multiple systems including ITPs, and deliver command files. ITPs may communicate with either logging system in case of failure of one, and are configured to primarily dial the primary logger, and after 5 failed attempts dial the backup logger. Five failed attempts at the backup logger reverts back to the primary logger, and so forth. The communication is via a conventional Rev 2 Hayes desktop modem connected to a dedicated analog phone line. Each receiving system logs data to a specific user directory structure using a Perl scripted logger program. Each ITP is assigned a username and password, although passwords are not necessary for logging operations as ITPs do not access the server directly but rather the logger script. The number of users, i.e. field systems, can be increased as needed with no change to the logger program, by simply adding users to each Linux system and configuring the field systems to dial-up and login as particular users.

Information is sent to the logger in 2048 byte packets. The logger computer reassembles the files from the packet format in which the files are transmitted. Data and engineering files from SURFCON are sent to the logger preceded with the 50 character PROCON metastructure and appended with the 70 character SURFCON metastructure (see Appendix G). Note that the 2 structures are identical for the first 49 characters. The duplication of metadata structures is a redundancy feature to assure that the description source of each file is embedded in that file and likely recoverable from at least one place or the other. The SURFCON metastructure does not include the terminating character '>'. Following the PROCON supplied file length, is the PROCON supplied CRC ( 9 characters including a trailing space) and SURFCON computed filelength (12 characters including a trailing space).

At each occasion that SURFCON makes an ISU connection, it transmits all files in its queue, and also requests a command list from the WHOI server. If such a command list exists on the server (typically composed manually by a person responsible for operation of the destination ITP), it is received by SURFCON and acknowledged by a short file (filename is of the form mmddhhnn.ACK), that echoes the command(s) received. A command list using the filename "outboundcmd" is composed and installed in the root directory of the particular ITP for which it is intended. Once transmitted, its name is appended with the date and time to show that it was transmitted and to prevent it from being re-transmitted. The formation of these files is simple and should be considered fixed field, i.e. additional white space characters are likely to cause problems. Command packets contain a string of commands, each followed by a $\langle\mathrm{CRLF}\rangle$. The form is CMND (upper or lower case), whitespace, parameters according to command. Parameters are separated by white space. Filenames normally include an 8 character name, '.' 
and 3 character extension, although filenames shorter than 8 chars may arise. Multiple instances of a given command can be used. EOTX terminates the command list. Following are the command packets that can presently be handled by SURFCON:

$\begin{array}{ll}\text { SFIL filename }<\text { CRLF }> & \text { Get specified file from SURFCON } \\ \text { SDIR directory }<\text { CRLF }> & \text { Get an ASCII file of the specified SURCON directory } \\ \text { SCMD command }<\text { CRLF }> & \text { Send SURFCON system command and execute } \\ \text { PFIL filename }<\text { CRLF }> & \text { Get specified file from PROCON } \\ \text { PDIR }<\text { CRLF }> & \text { Get a file of the PROCON directory.... up to } 50 \text { lines } \\ \text { PCDS }<\text { CRLF }> & \text { Get Profiler UIM status - not implemented. } \\ \text { EOTX }<\text { CRLF }> & \text { Terminates command list }\end{array}$

The SURFCON system commands that could be invoked remotely are:

$\begin{array}{ll}\text { COPY } & \text { source dest [/V] } \\ \text { DEL } & {[\text { drv:][pth][name] [/P }} \\ \text { ERASE } & {[\text { drv:][pth][name] [/P }} \\ \text { REN } & {[\mathrm{d}:][\text { p] oldname newname }} \\ \text { RESET } & \text { (hard reset) } \\ \text { SET } & \text { [var=[str]] [/SLFE?] }\end{array}$

SDIR, SFIL and SCMD commands are executed immediately upon receipt and if a file results, it is sent in the current session with the logger. SDIR requests a listing of the specified directory on the SURFCON. SFIL requests transmission of a specific filename. SCMD requests that the SURFCON execute the specified command as if it was executed on the command line. Obviously great care is required with such a brute force facility.

PDIR and PFIL commands are accepted and held for the next IM communications session with the PROCON, hence any results from such commands will at the earliest, be returned to the logger at the end of the next Profiler session. PDIR requests the PROCON directory list which typically has a few control and status files and the 40 most recent data \& engineering file pairs.

When a command packet is received by SURFCON, it is stored in a file with the extension *.ACK, and added to the transmission queue for the next cycle (to acknowledge receipt). Upon completion of the Iridium communications, commands for SURFCON are executed. Requested SURFCON files are added to the transmission queue for the next cycle, 
while SURFCON directory requests are stored in *.SDR files and added to the queue.

Commands for PROCON are relayed to PROCON and executed during the next IM cycle.

Requested PROCON files are added to the transmission queue for the next cycle, while PROCON directory requests are stored in *.PDR files and added to the queue.

Examples of logger command files:

PFIL LASTSENT.DAT

PFIL IRQ_XCPT.LOG

EOTX

PFIL DEPLOY.DAT

PDIR

SDIR c:llog

SFIL c:litpsys

EOTX

SCMD RESET

SCMD DEL c:I*.ACK

EOTX 


\begin{tabular}{|c|c|c|}
\hline $\begin{array}{l}\text { REPORT DOCUMENTATION } \\
\text { PAGE }\end{array}$ & 1. REPORT NO. ${ }_{\text {WHOI-2006-11 }}$ & 3. Recipient's Accession No. \\
\hline \multirow{2}{*}{\multicolumn{2}{|c|}{$\begin{array}{l}\text { 4. Title and Subtitle } \\
\text { Ice-Tethered Profilers for Real-Time Seawater Observations in the Polar } \\
\text { Oceans }\end{array}$}} & $\begin{array}{l}\text { 5. Report Date } \\
\text { June } 2006\end{array}$ \\
\hline & & 6. \\
\hline \multicolumn{2}{|c|}{$\begin{array}{l}\text { 7. Author(s) Krishfield, R., K. Doherty, D. Frye, T. Hammar, J. Kemp, D. Peters, } \\
\text { A.Proshutinsky. J.Toole and K. von der Hevdt }\end{array}$} & 8. Performing Organization Rept. No. \\
\hline \multirow{2}{*}{\multicolumn{2}{|c|}{$\begin{array}{l}\text { 9. Performing Organization Name and Address } \\
\text { Woods Hole Oceanographic Institution } \\
\text { Woods Hole, Massachusetts } 02543\end{array}$}} & 10. Project/Task/Work Unit No. \\
\hline & & $\begin{array}{l}\text { 11. Contract(C) or Grant(G) No. } \\
\text { (C) OCE-0324233 } \\
\text { (G) ARC-0519899 }\end{array}$ \\
\hline \multicolumn{2}{|c|}{ 12. Sponsoring Organization Name and Address } & 13. Type of Report \& Period Covered \\
\hline \multirow{2}{*}{\multicolumn{2}{|c|}{ National Science Foundation }} & Technical Report \\
\hline & & 14. \\
\hline
\end{tabular}

15. Supplementary Notes

This report should be cited as: Woods Hole Oceanog. Inst. Tech. Rept., WHOI-2006-11.

16. Abstract (Limit: 200 words)

An automated, easily-deployed Ice-Tethered Profiler (ITP) has been developed for deployment on perennial sea ice in polar oceans to measure changes in upper ocean temperature and salinity in all seasons. The ITP system consists of three components: a surface instrument that sits atop an ice floe, a weighted, plastic-jacketed wire-rope tether of arbitrary length (up to $800 \mathrm{~m}$ ) suspended from the surface instrument, and an instrumented underwater unit that profiles up and down the wire tether. The profiling underwater unit is similar in shape and dimension to an ARGO float except that the float's variable-buoyancy system is replaced with a traction drive unit. Deployment of ITPs may be conducted either from ice caps or icebreakers, utilizing a self contained tripod/winch system that requires no power. Careful selection of an appropriate multiyear ice floe is needed to prolong the lifetime of the system (up to 3 years depending on the profiling schedule). Shortly after deployment, each ITP begins profiling the water column at its programmed sampling interval. After each acquired temperature and salinity profile, the underwater unit (PROCON) transfers the data and engineering files using an inductive modem to the surface controller (SURFCON). SURFCON also accumulates battery voltages, buoy temperature, and locations from GPS at specified intervals in status files, and queues that information for transmission at the start of each new day. At frequent intervals, an Iridium satellite transceiver in the surface package calls and transmits queued status and CTD data files onto a WHOI logger computer, which are subsequently processed and displayed in near-real time at http://www.whoi.edu/itp. In 2004 and 2005, three ITP prototypes were deployed in the Arctic Ocean. Each system was programmed with accelerated sampling schedules of multiple one-way traverses per day between 10 and 750-760 m depth in order to quickly evaluate endurance and component fatigue. Two of the ITPs are continuing to function after more than 10 months and 1200 profiles. Larger motor currents are observed at times of fast ice floe motion when larger wire angles develop and drag forces on the profiler are increased. The CTD profile data so far obtained document interesting spatial variations in the major water masses of the Beaufort Gyre, show the double-diffusive thermohaline staircase that lies above the warm, salty Atlantic layer, and many mesoscale eddys. Deployed together with CRREL Ice Mass Balance (IMB) buoys, these ITP systems also operate as part of an Ice Based Observatory (IBO). Data returned from an array of IBOs within an Arctic Observing Network will provide valuable real time observations, support studies of ocean processes, and facilitate numerical model initialization and validation.

\section{Document Analysis a. Descriptors}

Arctic instrumentation

ocean profiler

ice-based observatory

b. Identifiers/Open-Ended Terms

c. COSATI Field/Group

18. Availability Statement

Approved for public release; distribution unlimited.

\begin{tabular}{|c|}
$\begin{array}{c}\text { 19. Security Class (This Report) } \\
\text { UNCLASSIFIED }\end{array}$ \\
\hline 20. Security Class (This Page)
\end{tabular}

21. No. of Pages 81

22. Price

OPTIONAL FORM 272 (4-77)

(Formerly NTIS-35)

Department of Commerce 